\title{
Highest Weight Modules and $b$-Functions of Semi-invariants
}

By

\author{
Akihiko GyoJA*
}

\section{§ 0 . Introduction}

0.1. In [8], we have given an irreducibility criterion for the generalized Verma modules in terms of the $b$-functions of semi-invariants. (See (2.4) for generalized Verma modules, (2.5) for semi-invariants, and (3.2) for $b$-functions.) Thus, in order to give explicit information about the irreducibility of the generalized Verma modules, it is necessary to calculate the $b$-functions of semiinvariants. The first purpose of this paper is to develop techniques to calculate them.

0.2. Our irreducibility criterion is far different from, and unfortunately, less complete than the one given by Jantzen [12], since we need to assume (at least) the anti-dominancy for a technical reason. Our second purpose is to formulate a conjecture, which would eliminate this undesirable assumption. (For the sake of simplicity, here in the introduction we restrict ourselves to those induced from one dimensional representations of the maximal parabolic subalgebras. See $\S 3$, especially (3.3), and $\S 9$ for the statement in its full generality.)

Conjecture. Let $\mathfrak{p}$ be a maximal parabolic subalgebra of a complex semisimple Lie algebra $\mathfrak{g}$, and $\varpi$ the unique fundamental weight which has an extension to the character of $\mathrm{p}$. Denote the extension by the same letter $w$. Let $f$ be the semi-invariant corresponding to $\varpi$, and $b(s)$ the b-function of $f$. For $\lambda \in \mathbf{C}$, the generalized Verma module $U(\mathfrak{g}) \otimes_{U(\mathfrak{p}) \cdot \dot{\lambda} \mathrm{W}} \mathbf{C}$ is irreducible if and only if $b(\lambda-j) \neq 0$ for any $j=1,2, \cdots$.

0.3. Let us consider the special case where $g$ is simple, the nilpotent radical $\mathfrak{u}$ of $\mathfrak{p}$ is commutative, a Levi subalgebra $\mathfrak{l}$ of $\mathfrak{p}$ is normalized by the

Communicated by M. Kashiwara, December 22, 1992.

1991 Mathematics Subject Classification: 22E47, 17 B35.

* Department of Fundamental Sciences, Faculty of Integrated Human Studies, Kyoto University, Kyoto 606-01, Japan. 
longest element of the Weyl group, and the generalized Verma module concerned is induced from a one dimensional representation. Let $G$ be a complex Lie group such that $\operatorname{Lie}(G)=\mathfrak{g}$ and $L$ the connected subgroup of $G$ with the Lie algebra $\mathrm{I}$. In this case, $(L, \mathrm{ad}, \mathfrak{u})$ is known to be an irreducible regular prehomogeneous vector space, and we can show that the $b$-function of the semi-invariant coincides with the $b$-function of the prehomogeneous vector space $(L$, ad, u) (cf. the proof of (4.2.1)). Thus, in this special case, the above conjecture asserts a relation between the irreducibility of certain generalized Verma modules and $b$-functions of the prehomogeneous vector space $(L, a d, \mathfrak{u})$. This relation can be proved by a case study, and was first observed by S. Suga [29]. In fact, it was the original motivation of the present work to explain and generalize this observation of Suga.

0.4. Besides the purposes stated above, it would be worth noting the importance of the microlocal analysis of semi-invariants in connection with other problems in the representation theory. For example, a conjecture of Kazhdan and Lusztig (see (6.2)) implies that the holonomy diagram (cf. (5.18)) would coincide with the $W$-graph of the regular representation given in [18] if $\mathfrak{g}$ is of type $A_{l}$ and $\mathfrak{p}$ is the Borel subalgebra. Thus the determination of the microlocal structure of semi-invariants remains an important problem, even if the $b$-function is determined.

0.5. This paper consists of nine sections. In $\S 1$, we review some known facts about $\mathscr{D}$-modules associated to complex powers of regular functions. In $\S 2$, we review some known facts about complex semisimple Lie algebras and Lie groups. In $\S 3$, we state our main conjectures concerning the scalar generalized Verma modules, namely the induced modules from one dimensional representations of parabolic subalgebras. In $\S 4$, first in (4.1), we observe that our conjectures hold for Verma modules. (This case is due to M. Kashiwara [15].) Next in (4.2)-(4.4), we show that our conjectures hold for commutative parabolic cases. We give two more examples (4.5) and (4.6). In $\S 5$, we give techniques to calculate $b$-functions of semi-invariants. In $\S 6$, we review the Kazhdan-Lusztig theory, and observe that it is useful for our calculation of $b$-functions. In $\S 7$ and $\S 8$, we calculate $b$-functions for some cases using the techniques given in $\S 5$ and $\S 6$. In $\S 9$, we discuss how to generalize the conjectures of $\S 3$ to non-scalar generalized Verma modules.

\section{§1. $\mathscr{D}$-modules Associated to Complex Powers of Functions}

1.1. Let $X$ be a connected smooth affine variety over the complex number field $\mathrm{C}, \mathcal{O}=\mathscr{O}_{X}$ the sheaf of regular functions, $\mathscr{D}=\mathscr{D}_{X}$ the sheaf of algebraic differential operators, $\mathscr{E}=\mathscr{E}_{X}$ the sheaf of micro-differential operators, $f_{1}, \cdots, f_{k} \in$ $\Gamma\left(X, \mathcal{O}_{X}\right) \backslash \mathbf{C}, g=\prod_{i=1}^{k} f_{i}, \Omega=X \backslash g^{-1}(0), \Omega^{\prime}$ some simply connected domain contained in $\Omega, \underline{\hat{\lambda}}=\left(\hat{\lambda}_{1}, \cdots, \hat{\lambda}_{k}\right) \in \mathbf{C}^{k}, \underline{\delta}=(1, \cdots, 1) \in \mathbf{C}^{k}$ and $\underline{f}^{\underline{\lambda}}+s \underline{\delta}$ a single 
valued branch of

$$
\Omega^{\prime} \times \mathbf{C} \ni(x, s) \longrightarrow \prod_{i=1}^{k} f_{i}(x)^{\lambda_{1}+s}=\prod_{i=1}^{k} f_{i}(x)^{\lambda_{1}} \cdot g(x)^{s} .
$$

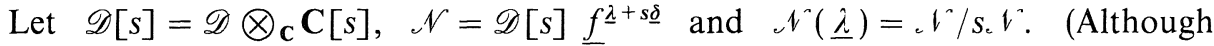
the function $\underline{f}^{\underline{\lambda}+s \underline{\delta}}$ is defined only on $\Omega^{\prime}$, the Zariski sheaves $\mathfrak{r}^{\prime}$ and $\boldsymbol{r}^{\wedge}(\underline{\lambda})$ are defined all over $X$.) Let $\underline{f}^{\underline{\lambda}+0 \underline{\delta}}:=\left(\underline{f}^{\underline{\lambda}+s \underline{\delta}} \bmod s_{\iota} \hat{\imath}^{\wedge}\right)$. Then $f^{\wedge}(\underline{\lambda})=$ $\mathscr{D} \underline{f}^{\underline{\lambda}+0 \underline{\delta}}$. Let $T^{*} X$ be the cotangent bundle of $X$,

$$
\begin{aligned}
& \mathbf{W}^{\prime}=\left\{(x, s \operatorname{grad} \log g(x)) \in T^{*} X \mid s \in \mathbf{C}^{\times}, g(x) \neq 0\right\}, \\
& \mathbf{W}=\mathbf{W}(g)=\mathbf{W}(g, X)=\text { the Zariski closure of } \mathbf{W}^{\prime}, \text { and } \\
& \mathbf{W}_{0}=\mathbf{W}_{0}(g)=\mathbf{W}_{0}(g, X)=\{(x, \xi) \in \mathbf{W}(g) \mid g(x) \xi=0\} .
\end{aligned}
$$

We denote by $\mathrm{Ch}(\mathscr{M})$ (resp. Ch $(\mathscr{M})$ ) the characteristic variety (resp. the characteristic cycle) of a coherent $\mathscr{D}$-module $\mathscr{M}$.

Lemma 1.2. The $\mathscr{D}_{X}$-module of (resp. $\left.\mathscr{N}(\underline{\hat{\lambda}})\right)$ is subholonomic (resp. holonomic). Moreover, $\mathrm{Ch}\left(\mathcal{N}^{\wedge}\right)=\mathbf{W}$ and the multiplicity of . $\hat{f}^{\wedge}$ along $\mathbf{W}$ is one.

A $\mathscr{D}_{X}$-module $\mathscr{M}$ is said to be holonomic (resp. subholonomic) if $\operatorname{dim} \mathrm{Ch}(\mathscr{M}) \leq \operatorname{dim} X$ (resp. $\leq \operatorname{dim} X+1$ ). This lemma can be proved in the same way as in [13].

Lemma 1.3. $[14,2.7]$. There exist a differential operator $P=P(s)=$ $P(s, \underline{f}, \underline{\lambda}) \in \Gamma\left(X, \mathscr{D}_{X}[s]\right)$ and a polynomial $b(s)=b(s, \underline{f}, \underline{\lambda}) \in \mathbf{C}[s] \backslash\{0\}$ such that

$$
P \underline{f}^{\underline{\lambda}+(s+1) \underline{\delta}}=b(s) \underline{f} \underline{\hat{\imath}}^{+s \underline{\delta}} .
$$

Lemma 1.4. [16, Lemma 2.3]. (Cf. [7, 2.3.8].) If $b(-j) \neq 0$ for $j=1,2, \cdots$, then $\mathcal{N}(\underline{\lambda})$ is naturally isomorphic to $\mathscr{f}(\underline{\lambda})\left[g^{-1}\right]$ with the natural $\mathscr{D}$-module structure.

Lemma 1.5. ([10,1.7]) (1) The characteristic cycle $\mathbf{C h} .1(\underline{\lambda})$ does not depend on $\underline{\lambda}$.

(2) The characteristic variety $\mathrm{Ch} .1(\underline{\lambda})$ is $\mathbf{W}_{0}(g)$.

\section{§2. Semi-invariants}

2.1. Let $G$ be a connected, simply connected, semisimple group over the complex number field $\mathbf{C}, B$ a Borel subgroup of $G, T$ a maximal torus contained 
in $B, B_{-}$the Borel subgroup such that $B \cap B_{-}=T, W=N_{G}(T) / T$, and $\mathfrak{g}, \mathfrak{b}, \mathfrak{b}_{-}$ and $\mathrm{t}$ the Lie algebras of $G, B, B_{-}$and $T$, respectively. Let $\mathrm{t}^{\vee}=\operatorname{Hom}_{\mathbf{C}}(\mathrm{t}, \mathbb{C})$, $R\left(\subset \mathrm{t}^{\vee}\right)$ the root system of $(\mathfrak{g}, \mathrm{t}), \mathfrak{g}(\alpha)$ the root subspace of $\mathfrak{g}$ corresponding to $\alpha \in R, R_{+}$the set of $\alpha \in R$ such that $\mathfrak{g}(\alpha) \subset \mathfrak{b}, R_{-}=-R_{+}, \rho=\frac{1}{2} \sum_{\alpha \in R_{+}} \alpha, \Pi=$ $\left\{\alpha_{1}, \cdots, \alpha_{l}\right\}$ the root basis contained in $R_{+}, \Pi^{\vee}=\left\{\alpha_{1}^{\vee}, \cdots, \alpha_{l}^{\vee}\right\}$ the set of simple coroots, and $\left\{\varpi_{1}, \cdots, \varpi_{l}\right\}$ (resp. $\left\{\varpi_{1}^{\vee}, \cdots, \varpi_{l}^{\vee}\right\}$ ) the set of fundamental weights (resp. fundamental coweights). Then $\alpha_{i}, \varpi_{i} \in \mathfrak{t}^{\vee}, \alpha_{i}^{\vee}, \varpi_{i}^{\vee} \in \mathfrak{t},\left\langle\alpha_{i}, \varpi_{j}^{\vee}\right\rangle=\delta_{i j}$ and $\left\langle\alpha_{i}^{\vee}, \varpi_{j}\right\rangle=\delta_{i j}$, where \langle\rangle denotes the natural pairing of a vector space and its dual. Concerning the numbering of simple roots, we follow [3, Planches I-IX]. For $\alpha \in R_{+}$, let $\alpha^{\vee}$ be the corresponding coroot, $r_{\alpha}$ the reflection with respect to $\alpha, r_{i}=r_{\alpha_{1}}$ and $S=\left\{r_{1}, \cdots, r_{l}\right\}$. Sometimes we write simply $i$ for $r_{i}$. The identity element of $G$ or $W$ is denoted by $e$, not by 1 . For $w \in W$, let $l(w)$ be its length with respect to $S$. Let $\geq$ denote the Bruhat order in $W$ so that the identity element $e$ becomes minimal. For each $\alpha \in R$, we fix a non-zero element $X_{\alpha}$ of $g(\alpha)$, which we shall call the root vector. For each $w \in W$, we fix its representative element in $N_{G}(T)(\subset G)$, which we shall denote by $\dot{w}$, or simply by $w$ if there is no fear of confusion. We denote the universal enveloping algebra by $U(-)$.

2.2. For a character, say $\lambda$, of $T$, we denote the corresponding character of $t$ by the same letter $\lambda$, and vice versa for a character of $t$ which can be integrated to a character of $T$. Thus we consider an element $\lambda$ of $\sum_{i=1}^{l} \mathbb{Z} \varpi_{i}$ as a character of $T$, which we shall denote by the same letter $\lambda$. We also denote the natural extension of the character $\lambda$ of $t$ (resp. $T$ ) to $\mathfrak{b}$ or $\mathfrak{b}_{-}$(resp. $B$ or $B_{-}$) by the same letter $\lambda$. Moreover, if $\lambda$ can be extended to a larger algebra (resp. group) containing $\mathfrak{b}$ or $\mathfrak{b}_{-}$(resp. $B$ or $B_{-}$), then we shall denote such extensions also by the same letter $\lambda$.

2.3. For a subset $I$ of $S$, let $W_{I}$ be the subgroup generated by $I, w_{I}$ the longest element of $W_{I}, I^{\prime}=w_{S} I w_{S}(\subset S), \quad \Pi_{I}=\left\{\alpha \in \Pi \mid r_{\alpha} \in I\right\}, \quad R_{I}$ the root subsystem of $R$ generated by $\Pi_{I}, R_{I, \pm}=R_{I} \cap R_{ \pm}, \quad \mathfrak{l}=\mathfrak{l}(I)=\mathfrak{t}+\sum_{\alpha \in R_{I}} \mathfrak{g}(\alpha)$, $\mathfrak{u}_{ \pm}=\mathfrak{u}_{ \pm}(I)=\sum_{\alpha \in R_{ \pm} \backslash R_{I}} \mathfrak{g}(\alpha)$, and $\mathfrak{p}_{ \pm}=\mathfrak{p}_{ \pm}(I)=\mathrm{I}+\mathfrak{u}_{ \pm}$. We denote the connected subgroups of $G$ corresponding to $I, \mathfrak{u}_{ \pm}$and $\mathfrak{p}_{ \pm}$by $L=L(I), U_{ \pm}=U_{ \pm}(I)$ and $P_{ \pm}=P_{ \pm}(I)$, respectively. We usually write $\mathfrak{p}=\mathfrak{p}(I)$ and $P=P(I)$ for $\mathfrak{p}_{+}=\mathfrak{p}_{+}(I)$ and $P_{+}=P_{+}(I)$. Put $P^{\prime}=P\left(I^{\prime}\right)$ etc. For subsets $J$ and $K$ of $S$, let $\left(W_{J} \backslash W / W_{K}\right)_{s}$ (resp. $\left.\left(W_{J} \backslash W / W_{K}\right)_{l}\right)$ be the representatives of $W_{J} \backslash W / W_{K}$ consisting of the shortest (resp. longest) element in each double coset. We write $\left(W / W_{K}\right)_{s}$ etc. for $\left(W_{\phi} \backslash W / W_{K}\right)_{s}$ etc.

2.4. Generalized Verma module Let us fix a subset $I$ of $S$ and let $\mathfrak{p}=\mathfrak{p}(I)$ etc. Let $\lambda$ be a character of $\mathrm{t}$ such that $\left\langle\lambda, \alpha^{\vee}\right\rangle \in \mathbf{Z}_{\geq 0}$ for any $\alpha \in \Pi_{I}$. Then $\lambda$ is the highest weight of a finite dimensional irreducible $\mathfrak{p}$-module 
$V(\lambda)=V(\lambda, \mathfrak{p}) . \quad$ The $U(\mathfrak{g})$-module $M(\lambda)=M(\lambda, \mathfrak{p})=U(\mathfrak{g}) \otimes_{U(\mathfrak{p})} V(\lambda, \mathfrak{p})$ is called a generalized Verma module.

2.5. Semi-invariant Let $\lambda$ be an integral dominant weight, i.e., a character of $\mathrm{t}$ such that $\left\langle\lambda, \alpha^{v}\right\rangle \in \mathbf{Z}_{\geq 0}$ for any $\alpha \in \Pi$. Then there exists a uniquely determined regular function $f^{\lambda}$ on $G$ such that $f^{\lambda}\left(\dot{w}_{S}\right)=1$, and $f^{\lambda}\left(b x b^{\prime}\right)=$ $\left(w_{s} \lambda\right)(b) \lambda\left(b^{\prime}\right) f^{\lambda}(x) \quad\left(b, b^{\prime} \in B, x \in G\right)$, which we shall call the semi-invariant corresponding to $\lambda$. The semi-invariant can be constructed as follows. Let $V(\lambda)$ be the irreducible $g$-module with the highest weight $\lambda, v(\lambda)$ a highest weight vector, $V(\lambda)^{\vee}=V\left(-w_{s} \lambda\right)$ the dual of $V(\lambda),\langle>$ the natural pairing of $V(\lambda)$ and $V(\lambda)^{\vee}$, and $v(-\lambda)$ a lowest weight vector of $V(\lambda)^{\vee}$. Normalize $v(-\lambda)$ so that $\langle v(-\lambda) \mid v(\lambda)\rangle=1$. Then $f^{\lambda}(x)=\left\langle v(-\lambda) \mid \dot{w}_{S}^{-1} x v(\lambda)\right\rangle$. Put $f_{i}=f^{\sigma_{l}}$. Then, for $\lambda=\sum_{i=1}^{l} \lambda_{i} \varpi_{i}\left(\lambda_{i} \in \mathbf{Z}_{\geq 0}\right)$, we have $f^{\lambda}=\prod_{i=1}^{l} f_{i}^{\lambda_{z}}$. More generally, we put $f^{\lambda}=\prod_{i=1}^{l} f_{i}^{\lambda_{2}}$ for $\lambda=\sum_{i=1}^{l} \lambda_{i} \varpi_{i}\left(\lambda_{i} \in \mathbf{C}\right)$. We understand $(\lambda, x) \rightarrow f^{\lambda}(x)$ as a single valued branch on $C^{l} \times \Omega^{\prime}$, where $\Omega^{\prime}$ is some simply connected domain contained in $\bigcap_{i=1}^{l} f_{i}^{-1}\left(\mathbf{C}^{\times}\right)$. Sometimes, it is convenient to consider $f_{i}^{\prime}(x):=$ $f_{i}\left(\dot{w}_{S} x\right)$ and $f^{\prime \lambda}(x):=f^{\lambda}\left(\dot{w}_{S} x\right)$ instead of $f_{i}$ and $f^{\lambda}$, which we shall also call the semi-invariants if there is no fear of confusion. They satisfy $f^{\prime \lambda}(e)=1$ and $f^{\prime \lambda}\left(b^{\prime} x b\right)=\lambda\left(b^{\prime}\right) \lambda(b) f^{\prime \lambda}(x)$ for $b^{\prime} \in B_{-}, x \in G$ and $b \in B$.

Lemma 2.6. ([8, 9.9 and 9.10]) (1) $A$ defining equation of the subvariety $\overline{B w_{S} r_{i} B}$ of $G$ is given by $f_{i}=0$. (2) $G-P^{\prime} w_{S} P=\bigcup_{i \in S-I} \overline{B w_{S} r_{i} B}$. (3) For any $w \in\left(W_{I^{\prime}} \backslash W / W_{I}\right)_{l}-\left\{w_{S}\right\}, \prod_{i \in S-I} f_{i} \equiv 0$ on $\overline{B w B}$.

Lemma 2.7. ([8, 9.11]) The rational characters $\varpi_{i}$ and $w_{S} \varpi_{i}(i \in I)$ of $B$ can be extended to those of $P=P(I)$ and $P^{\prime}=P\left(I^{\prime}\right)=P\left(w_{S} I w_{S}\right)$, respectively, and we have $f_{i}\left(p^{\prime} x p\right)=\left(w_{s} \varpi_{i}\right)\left(p^{\prime}\right) \varpi_{i}(p) f_{i}(x)$ for $p^{\prime} \in P^{\prime}, x \in G$ and $p \in P$.

\section{§3. Conjectures}

3.1. Fix a subset $I$ of $S$. Let $S-I=\left\{i_{1}, \cdots, i_{k}\right\}$ and $I=\left\{i_{k+1}, \cdots, i_{l}\right\}$. (Here we used the convention " $r_{i}=i$ ".) Let $s_{i_{1}}, \cdots, s_{i_{k}}$ be independent complex variables, $\underline{s}=\sum_{i \in S-I} s_{i} \varpi_{i}, \delta=\sum_{i \in S-I} \varpi_{i}$, and $\mathbf{C}[\underline{s}]=\mathbf{C}\left[s_{i_{1}}, \cdots, s_{i_{k}}\right]$. Let $s$ be another complex variable, $\mathscr{N}=\mathscr{D}_{G}[s] f^{\lambda+s \delta}\left(\lambda \in \sum_{i \in S-I} \mathbf{C} \varpi_{i}\right), \mathscr{N}(\hat{\lambda})=\mathcal{L}^{\hat{\gamma}} / s_{\mathcal{L}} \hat{\hat{N}}$, and $f^{\lambda+0 \delta}:=\left(f^{\lambda+s \delta} \bmod s \mathcal{N}\right)$. Then $\mathcal{N}(\lambda)=\mathscr{D}_{G} f^{\lambda+0 \delta}$. Note that $f^{\lambda+0 \delta}=$ $\underline{f} \underline{\underline{\lambda}}+\underline{\underline{\delta}}$ in the notation of $\S 1$, where $\underline{\delta}=(1, \cdots, 1), \underline{f}=\left(f_{i_{1}}, \cdots, f_{i_{k}}\right)$, and $\underline{\lambda}=\left(\lambda_{i_{1}}, \cdots, \lambda_{i_{k}}\right)$.

Conjecture A. For $\mu \in \sum_{i \in S-I} \mathbf{Z}_{\geq 0} \varpi_{i}$, there exist a differential operator $P_{\mu} \in \Gamma\left(G, \mathscr{D}_{G}\right)$, a non-singular point $p \in \mathbf{W}_{0}=\mathbf{W}_{0}\left(f^{\delta}\right)$ independent of $\mu, a$ micro-differential operator $Q_{\mu}(\underline{s}) \in \mathscr{E}_{G . p}[\underline{s}]$ whose principal symbol is independent of $\underline{s}$ and invertible at $p$, and a non-zero polynomial $b_{\mu}(\underline{s}) \in \mathbf{C}[\underline{s}]$ such that 
$P_{\mu} f^{\lambda+\mu+0 \delta}=b_{\mu}(\lambda) f^{\lambda+0 \delta}$ and $f^{\lambda+\mu+0 \delta}=b_{\mu}(\lambda) Q_{\mu}(\lambda) f^{\lambda+0 \delta}$ for any $\lambda \in \sum_{i \in S-I} \mathbf{C} \varpi_{i}$.

Remark 3.2. By $[10,6.4]$, the polynomials $b_{\mu}(\underline{s})$ are uniquely determined up to non-zero constant multiple. We call the polynomials $b_{\mu}(\underline{s})$ the $b$ functions.

3.3. Assuming Conjecture $A$, we make the following conjectures.

Conjecture B (Main Conjecture). The generalized Verma module $M(\lambda, \mathfrak{p}(I))$ $\left(\lambda \in \sum_{i \in S-I} \mathbf{C} \varpi_{i}\right)$ is irreducible if and only if $b_{\mu}(\lambda-\mu) \neq 0$ for any $\mu \in \sum_{i \in S-I} \mathbf{Z}_{\geq 0} \varpi_{i}$.

Conjecture C. The following conditions are equivalent for $\lambda \in \sum_{i \in S-I} \mathbf{C} \varpi_{i}$. (1) $\left\langle\lambda+\rho, \alpha^{\vee}\right\rangle \neq 0,-1,-2, \cdots$ for any $\alpha \in R_{+}$. (2) $b_{\mu}(\lambda) \neq 0$ for any $\mu \in$ $\sum_{i \in S-I} \mathbf{Z}_{\geq 0} \varpi_{i}$

3.4. It is easy to see that Conjectures $A, B$ and $C$ are equivalent to the following Conjectures $A^{\prime}, B^{\prime}$ and $C^{\prime}$, respectively. (Conjectures $B^{\prime}$ and $C^{\prime}$ have meaning under Conjecture $A^{\prime}$.)

Conjecture $\mathbf{A}^{\prime}$. For any $i \in S-I$, there exist a differential operator $P_{i} \in \Gamma\left(G, \mathscr{D}_{G}\right)$, a point $p \in \mathbf{W}_{0}=\mathbf{W}_{0}\left(f^{\delta}\right)$ independent of $i$, a micro-differential operator $Q_{i}[\underline{s}] \in \mathscr{E}_{G, p}[\underline{s}]$ whose principal symbol is independent of $\underline{s}$ and invertible at $p$, and a non-zero polynomial $b_{i}(\underline{s}) \in \mathbf{C}[\underline{s}]$ such that $P_{i} f^{\lambda+\sigma_{1}+0 \delta}=$ $b_{i}(\lambda) f^{\lambda+0 \delta}$, and $f^{\lambda+\varpi_{i}+0 \delta}=b_{i}(\lambda) Q_{i}(\lambda) f^{\lambda+0 \delta}$ for any $\lambda \in \sum_{i \in S-I} \mathbf{C} \varpi_{i}$.

Conjecture B'. The generalized Verma module $M(\lambda, \mathfrak{p}(I))\left(\lambda \in \sum_{i \in S-I} \mathbf{C} \varpi_{i}\right)$ is irreducible if and only if $b_{i}\left(\lambda-\varpi_{i}-\mu\right) \neq 0$ for any $i \in S-I$ and $\mu \in \sum_{i \in S-I} \mathbf{Z}_{\geq 0} \varpi_{i}$.

Conjecture $\mathbf{C}^{\prime}$. The following conditions are equivalent for $\lambda \in \sum_{i \in S-I} \mathbf{C} \varpi_{i}$. (1) $\left\langle\lambda+\rho, \alpha^{\vee}\right\rangle \neq 0,-1,-2, \cdots$ for any $\alpha \in R_{+}$. (2) $b_{i}(\lambda+\mu) \neq 0$ for any $i \in S-I$ and $\mu \in \sum_{i \in S-I} \mathbf{Z}_{\geq 0} \varpi_{i}$.

Remark 3.5. Let us show that $\mathbf{W}_{0}$ in $[\mathbf{8}, 9.12]$ coincides with $\mathbf{W}_{0}\left(f^{\delta}\right)$. Let $\lambda=\sum_{i \in S-I} \lambda_{i} \varpi_{i}$. Then, on the open set $\left(f^{\delta}\right)^{-1}\left(\mathbf{C}^{\times}\right)=\bigcap_{i \in S-I} f_{i}^{-1}\left(\mathbf{C}^{\times}\right), \mathscr{N}^{\prime}(\lambda)$ in $[8,9.12]$ is naturally isomorphic to $\mathscr{N}(\lambda)$ defined in (3.1). Hence $\mathscr{N}^{\prime}(\lambda)\left[f_{i_{1}}^{-1}, \cdots, f_{i_{k}}^{-1}\right]$ in $[8]$ is isomorphic to $\mathcal{N}(\lambda)\left[\left(f^{\delta}\right)^{-1}\right]$. But, by (1.4) and (1.5), we have $\mathrm{Ch} \mathscr{N}(\hat{\lambda})=\mathrm{Ch} \mathscr{N}(\hat{\lambda})\left[\left(f^{\delta}\right)^{-1}\right]=\mathbf{W}_{0}\left(f^{\delta}\right)$. Hence the characteristic variety of $\mathscr{N}^{\prime}(\lambda)\left[f_{i_{1}}^{-1}, \cdots, f_{i_{k}}^{-1}\right]$ is $\mathbf{W}_{0}\left(f^{\delta}\right)$. Thus Conjecture $A$ would imply that the assumptions $(9.12 .3)$ and $(9.12 .4)$ of $[8]$ are always satisfied.

\section{§4. Examples (1)}

4.0. Here we calculate $b$-functions of semi-invariants for some $(\mathfrak{g}, I)$. If $\mathfrak{g}$ is of type $X_{l}$ and $S-I=\left\{i_{1}, \cdots, i_{k}\right\}$, we shall denote such a pair by 
$\left(X_{l}, i_{1}, \cdots, i_{k}\right)$, and indicate it graphically by colouring black the vertexes corresponding to $I$ of the Dynkin diagram of $\mathfrak{g}$. Along with the calculation of $b$-functions, we also determine the set of $\lambda \in \sum_{i \in S-I} \mathbf{C} \varpi_{i}$ such that $M(\lambda, \mathfrak{p}(I))$ is reducible by applying the irreducibility criterion of Jantzen [12], except for (4.1). In (4.1), we consider the case where $I=\phi$. The content of (4.1) is a restatement of [15]. In (4.2)-(4.4), we consider the case where $\mathfrak{p}$ is a maximal parabolic subalgebra and its nilpotent radical is commutative. In (4.5) and (4.6), we study two examples, where the $b$-functions can be calculated directly.

4.1. Verma modules Let us consider the case where $I=\phi$. In this case, $\mathfrak{p}=\mathfrak{b}$ and $M(\lambda, \mathfrak{p})$ is the Verma module.

Lemma 4.1.1. [15, Theorem 2.1 and Remark 2.3] For any $\mu \in \sum_{i \in S} \mathbf{Z}_{\geq 0} \varpi_{i}$, there exist a differential operator $P_{\mu} \in \Gamma\left(G, \mathscr{D}_{G}\right)$ and an invertible micro-differential operator $Q_{\mu}$ in a neighbourhood of a generic point of the conormal bundle of $B$ in $G$, such that $P_{\mu} f^{\lambda+\mu+0 \delta}=b_{\mu}(\lambda) f^{\lambda+0 \delta}$ and $Q_{\mu} f^{\lambda+\mu+0 \delta}=b_{\mu}(\lambda) f^{\lambda+0 \delta}$ with $b_{\mu}(\lambda)=$ $\prod_{\alpha \in R_{+}}\left[\left\langle\lambda+\rho, \alpha^{\vee}\right\rangle\right]^{\langle\mu, \alpha v\rangle}$, where $[x]^{0}=1$ and $[x]^{m}=x(x+1) \cdots,(x+m-1)$ for $m>0$.

Lemma 4.1.2. ( $[6,7.6 .24])$ The following conditions are equivalent for $\lambda \in \mathrm{t}^{\vee}$. (1) The Verma module $M(\lambda, \mathfrak{b})$ is irreducible. (2) $\left\langle\lambda+\rho, \alpha^{\vee}\right\rangle \neq 1,2, \cdots$ for any $\alpha \in R_{+}$. (3) $b_{\mu}(\lambda-\mu) \neq 0$ for any $\mu \in \sum_{i \in S} \mathbf{Z}_{\geq 0} \varpi_{i}$.

Lemma 4.1.3. The following conditions are equivalent for $\lambda \in \mathrm{t}^{\vee}$. $\left\langle\lambda+\rho, \alpha^{v}\right\rangle \neq 0,-1,-2 \cdots$, for any $\alpha \in R_{+}$. (2) $b_{\mu}(\lambda) \neq 0$ for any $\mu \in$ $\sum_{i \in S} \mathbf{Z}_{\geq 0} \varpi_{i}$

The verification of $(2) \Leftrightarrow(3)$ in (4.1.2), and (4.1.3) is easy and omitted. Thus Conjectures $A, B$ and $C$ hold for this case.

4.2. Commutative parabolic cases (1) Let us consider the case where $S-I$ consists of only one simple reflection $r_{i}$ and the coefficient of $\alpha_{i}$ in the highest root is equal to one. Then the nilpotent radical of the parabolic subalgebra $\mathfrak{p}(I)$ is commutative, and all such parabolic subalgebras can be obtained in this way. We refer to these cases as commutative parabolic cases. Further we assume that $\mathfrak{g}$ is simple. Such $(\mathfrak{g}, i)$ can be classified as follows:

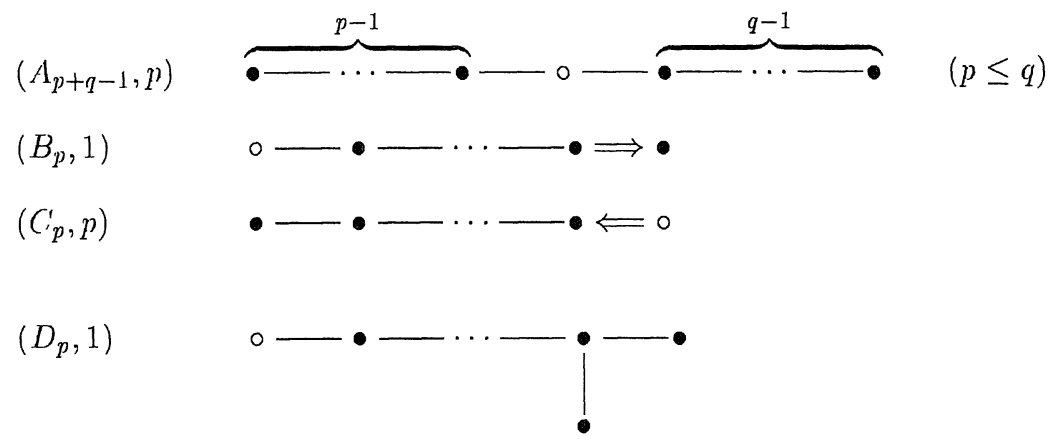




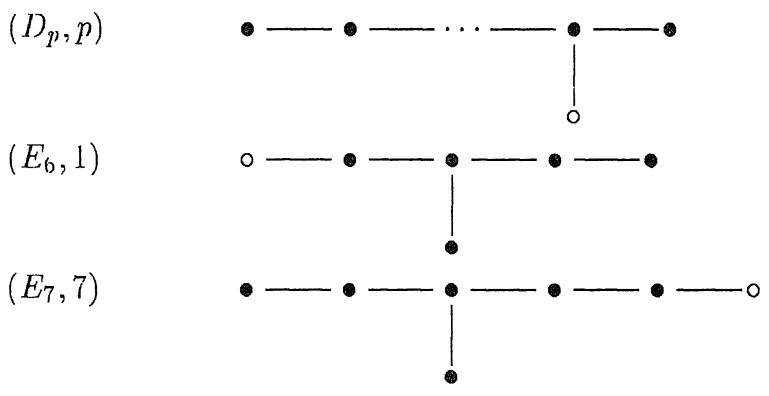

(Figure 1)

(Note that $\left(A_{p+q-1}, p\right) \simeq\left(A_{p+q-1}, q\right),\left(D_{p}, p\right) \simeq\left(D_{p}, p-1\right)$ and $\left(E_{6}, 1\right) \simeq\left(E_{6}, 6\right)$.)

First, in this number, we consider the cases where $-w_{S}\left(\alpha_{i}\right)=\alpha_{i}$. We shall refer to these cases as regular commutative parabolic cases. It is known that $\left(L(I)\right.$, ad, $\left.\mathfrak{u}_{-}(I)\right) \simeq\left(L(I)\right.$, ad, $\left.U_{-}(I)\right)$ is an irreducible regular prehomogeneous vector space. (See [25] and [7] for prehomogeneous vector spaces. See [22] and [21] for the prehomogeneous vector spaces of this special type.) The regular commutative parabolic cases are $\left(A_{2 p-1}, p\right),\left(B_{p}, 1\right),\left(C_{p}, p\right),\left(D_{p}, 1\right)$, $\left(D_{2 p}, 2 p\right)$ and $\left(E_{7}, 7\right)$.

Lemma 4.2.1. Conjecture $A$ holds for the regular commutative parabolic cases.

Proof. Let $b_{i}(s)$ be the minimal polynomial of $s \in \operatorname{End}_{\mathscr{D}_{G}}\left(\mathscr{D}_{G}[s] f_{i}^{s} /\right.$ $\left.\mathscr{D}_{G}[s] f_{i}^{s+1}\right)$. Since every $B \times B$-orbit contains the identity element $e$ in its closure, $b_{i}(s)$ is also the minimal polynomial of $s \in \operatorname{End}_{\mathscr{D}_{0}}\left(\mathscr{D}_{0}[s] f_{i}^{s} / \mathscr{D}_{0}[s] f_{i}^{s+1}\right)$, where $\mathscr{D}_{0}$ denotes the stalk of $\mathscr{D}_{G}$ at $e$. (Cf. the proof of $[7,2.5 .3]$.) Since $U_{-} \cdot P$ is an open neighbourhood of $e$ and $f_{i}(u p)=\varpi_{i}(p) f_{i}(u)$ for any $u \in U_{-}$ and $p \in P$, in order to prove Conjecture $A$, it suffices to show the existence of a micro-differential operator $Q \in \mathscr{E}_{U_{-}}$such that $Q\left(f_{i} \mid U_{-}\right)^{s+1}=b_{i}(s)\left(f_{i} \mid U_{-}\right)^{s}$ and invertible at some point of $\mathbf{W}_{0}\left(f_{i} \mid U_{-}, U_{-}\right)$. (Recall the convention (2.2) and note that $\varpi_{i}(p) \neq 0$ for any $p \in P$.) By the definition of semi-invariants,

$$
f_{i}\left(l u l^{-1}\right)=\left(w_{S} \varpi_{i}-\varpi_{i}\right)(l) f_{i}(u)=\left(-2 \varpi_{i}\right)(l) f_{i}(u)
$$

for any $l \in L$ and $u \in U_{-}$, i.e., $\left(f_{i} \circ \exp \right) \mid u_{-}$is a relative invariant of the regular prehomogeneous vector space $\left(L, \mathrm{ad}, \mathfrak{u}_{-}\right)$. Hence we know the existence of the desired micro-differential operator $[24,4.6]$.

Remark 4.2.3. The relative invariant $\left(f_{i} \circ \exp \right) \mid \mathfrak{u}_{-}$appeared in $[22$, Theorem 1.4.2].

4.2.4. Let us determine the explicit form of $b_{i}(s)$. By (4.2.2), $\left(f_{i}\right.$ 。 exp $) \mid \mathfrak{u}_{-}$ is a relative invariant corresponding to the character $-2 \varpi_{i}$. Since $\operatorname{ad}\left(-\varpi_{i}^{v}\right)$ $\equiv 1$ on $\mathfrak{u}_{-}$, the polynomial degree of $\left(f_{i} \circ \exp \right) \mid \mathfrak{u}_{-}$is equal to $2\left\langle\varpi_{i}, \varpi_{i}^{\vee}\right\rangle$, 
which is equal to $p, 2, p, 2, p, 3$ for the cases $\left(A_{2 p-1}, p\right),\left(B_{p}, 1\right),\left(C_{p}, p\right),\left(D_{p}, 1\right)$, $\left(D_{2 p}, 2 p\right),\left(E_{7}, 7\right)$, respectively. Comparing with the degree of irreducible relative invariants of prehomogeneous vector spaces $\left(L, \mathrm{ad}, \mathfrak{u}_{-}\right)$[25], we can see that each $\left(f_{i} \circ \exp \right) \mid \mathfrak{u}_{-}$is an irreducible relative invariant. As is seen from the proof of $(4.2 .1), b_{i}(s)$ is equal to the $b$-function of $\left(f_{i} \circ \exp \right) \mid \mathfrak{u}_{-}$, whose explicit form is given by

$$
\begin{array}{ll}
\left(A_{2 p-1}, p\right) & b_{p}(s)=(s+1)(s+2) \cdots(s+p) \\
\left(B_{p}, 1\right) & b_{1}(s)=(s+1)\left(s+\frac{2 p-1}{2}\right) \\
\left(C_{p}, p\right) & b_{p}(s)=(s+1)\left(s+\frac{3}{2}\right)\left(s+\frac{4}{2}\right) \cdots\left(s+\frac{p+1}{2}\right) \\
\left(D_{p}, 1\right) & b_{1}(s)=(s+1)\left(s+\frac{2 p-2}{2}\right) \\
\left(D_{2 p}, 2 p\right) & b_{2 p}(s)=(s+1)(s+3) \cdots(s+2 p-1) \\
\left(E_{7}, 7\right) & b_{7}(s)=(s+1)(s+5)(s+9)
\end{array}
$$

See [20], also [21] and [11].

Lemma 4.2.5. Conjecture $B$ holds for the regular commutative parabolic cases.

We can check this assertion by a direct calculation using the irreducibility criterion of Jantzen [12]. This assertion is essentially due to S. Suga [29], and is the original motivation of the present work as is explained in (0.3). It is easy to see that Conjecture $C$ also holds for the regular commutative parabolic cases.

4.3. $\left(E_{6}, 1\right)$

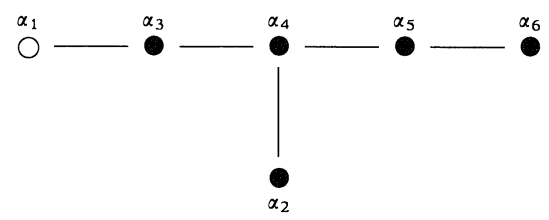

Let $G$ be a complex Lie algebra of type $E_{6}, \mathfrak{g}=\operatorname{Lie}(G), I=S-\left\{r_{1}\right\}$, $I^{\prime}=w_{S} I w_{S}, P=P(I), P^{\prime}=P\left(I^{\prime}\right)$ and $L^{\prime}=L\left(I^{\prime}\right)$. Put $J=\{1,2,3,4,5\}$.

Lemma 4.3.1. $w_{S} \in P_{-} \cdot L(J) P$.

Proof. It suffices to show that $w_{S} \in w_{S} W_{I} \cdot w_{S} \cdot W_{J} W_{I}$, or equivalently that

$$
w_{S} \in W_{I} W_{J} W_{I} \text {. }
$$

We can show that the coset representatives in $\left(W_{I} \backslash W\right)_{s}$ are given by the 


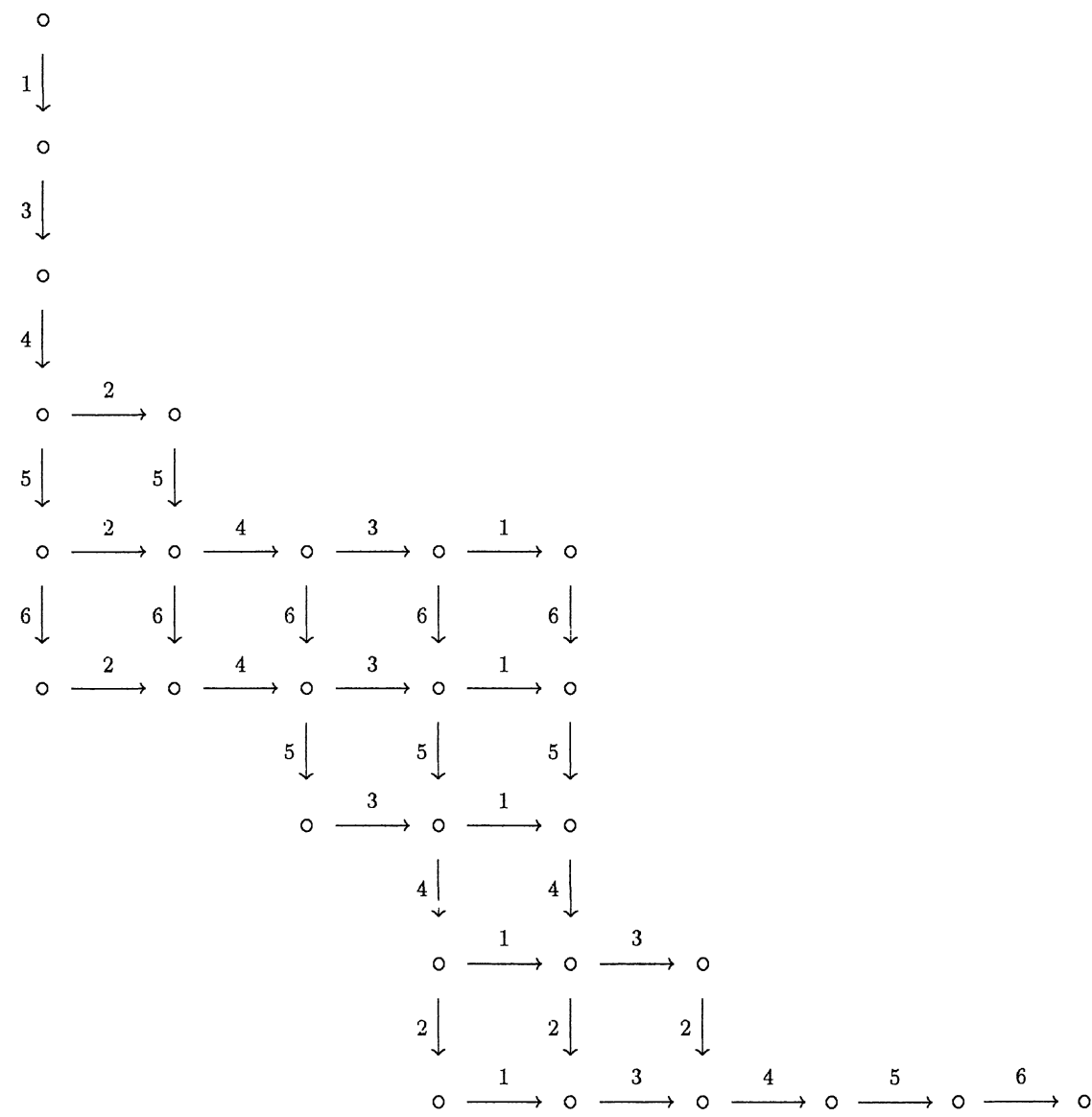

(Figure 2)

diagram of Figure 2.

(It implies that $\left(W_{I} \backslash W\right)_{s}=\{e, 1,13,134,1342,1345,13425, \cdots\}$ and all the expressions of elements of $\left(W_{I} \backslash W\right)_{s}$ obtained in this way are reduced.) Hence $w_{S}=w_{I} \cdot 13425431 \cdot 65432456 \in W_{I} W_{J} W_{I}$.

Lemma 4.3.3. The morphism $\mu: P_{-} \times L(J) \times P \rightarrow G$ defined by the multiplication is a submersion.

Proof. It is enough to show the surjectivity of $d \mu$ at $(e, w, e)$ for $w \in W_{J}$. Hence it suffices to show that $\mathfrak{p}_{-}+\mathfrak{l}(J)+(\operatorname{ad} w) \mathfrak{p}=\mathfrak{g}$, or equivalently that $\left(R_{-} \cup R_{I}\right) \cup R_{J} \cup w\left(R_{+} \cup R_{I}\right)=R$. Since $w \in W_{J}$, the left hand side contains $R_{-} \cup R_{J} \cup w R_{+} \supset R_{-} \cup R_{J} \cup\left(R_{+} \backslash R_{J}\right)=R$.

Lemma 4.3.4. The morphism $\mu: P_{-} \times L(J) \times P \rightarrow G$ is smooth and surjective.

Proof. By (4.3.3), $\mu$ is smooth and its image $G_{0}$ is an open set of 
$G$. Hence $G-G_{0}$ is a $P_{-} \times P$-stable, closed subset of $G$ which does not contain $w_{S}$ (cf. (4.3.1)). Since every coset $P_{-} \cdot g P(g \in G)$ contains $w_{S}$ in its closure, $G-G_{0}$ is empty, i.e., $\mu$ is surjective.

Lemma 4.3.5. (1) Conjecture $A$ holds for $\left(E_{6}, 1\right)$. (2) $b_{1}(s)=(s+1)(s+4)$.

Proof. Let $f_{i}^{\prime}(x)=f_{i}\left(w_{S} x\right) . \quad$ By $(2.7), \mu^{*} f_{1}^{\prime}=\varpi_{1} \otimes\left(f_{1}^{\prime} \mid L(J)\right) \otimes \varpi_{1} . \quad$ As is easily seen, $\left(f_{1}^{\prime} \mid L(J)\right)\left(w_{J}^{-1} x\right)$ is the semi-invariant of $L(J)$ corresponding to the following white node.

$\left(D_{5}, 1\right)$

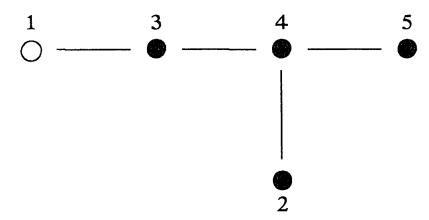

By (4.3.4), we can reduce the proof to the case $\left(D_{5}, 1\right)$, which we have already taken up in (4.2).

\subsection{Commutative parabolic cases (2)}

Lemma 4.4.1. (1) Conjectures $A, B$ and $C$ hold for commutative parabolic cases. (2) The b-functions are given by

$$
\begin{array}{ll}
\left(A_{p+q-1}, p\right) & b_{p}(s)=(s+1)(s+2) \cdots(s+p) \quad(p<q) \\
\left(D_{2 p+1}, 2 p+1\right) & b_{2 p+1}(s)=(s+1)(s+3) \cdots(s+2 p-1) \\
\left(E_{6}, 1\right) & b_{1}(s)=(s+1)(s+4) .
\end{array}
$$

See (4.2.4) for the $b$-functions in the regular commutative parabolic cases.

Proof. We shall reduce the proof to the regular commutative parabolic cases as in (4.3). More precisely, by showing (4.3.2) for some $J$, we shall reduce the proof as follows; $\left(A_{p+q-1}, p\right) \Rightarrow\left(A_{2 p-1}, p\right)(p<q)$, and $\left(D_{2 p+1}, 2 p+1\right) \Rightarrow$ $\left(D_{2 p}, 2 p\right)$.

For the case $\left(A_{p+q-1}, p\right)(p<q)$, let $J=\{1,2, \cdots, 2 p-1\}$, and $K=$ $\{2 p+1, \cdots, p+q-1\}(\subset I)$. Calculating products as permutations, we get $w_{I} w_{S} w_{I}=w_{S-\{2 p\}}$, and hence $w_{S}=w_{I} w_{S-\{2 p\}} w_{I}=w_{I} w_{J} w_{K} w_{I} \in W_{I} W_{J} W_{I} . \quad$ (If $q=$ $p+1$, then $K=\phi$ and $w_{K}=e$. Recall that $I=S-\{p\}$ in the present case.)

For the case $\left(D_{2 p+1}, 2 p+1\right)$, let $J=S-\{1\}$. Then $w_{S}=w_{I} w_{J} w_{I} \in$ $W_{I} W_{J} W_{I}$. In fact, in the notation of [3, Planche IV],

$$
\begin{aligned}
& \varepsilon_{i} \stackrel{w_{I}}{\longrightarrow} \varepsilon_{2 p+2-i} \stackrel{w_{J}}{\longrightarrow}-\varepsilon_{2 p+2-i} \stackrel{w_{I}}{\longrightarrow}-\varepsilon_{i} \quad(i \neq 2 p+1), \text { and } \\
& \varepsilon_{2 p+1} \stackrel{w_{I}}{\longrightarrow} \varepsilon_{1} \stackrel{w_{J}}{\longrightarrow} \varepsilon_{1} \stackrel{w_{I}}{\longrightarrow} \varepsilon_{2 p+1} .
\end{aligned}
$$


4.5. $\left(C_{n}, 1\right)$

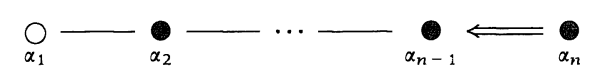

Let

$$
\begin{aligned}
& K=K_{n}=\left(\begin{array}{cc} 
& 1 \\
1 &
\end{array}\right) \in G L_{n}(\mathbf{C}), \quad J=\left(\begin{array}{rr} 
& K \\
-K &
\end{array}\right), \\
& \mathfrak{g}=\mathfrak{s p}_{2 n}(\mathbf{C})=\left\{X \in M_{2 n}(\mathbf{C}) \mid X J+J^{t} X=0\right\}, \\
& G=S p_{2 n}(\mathbf{C})=\left\{g \in G L_{2 n}(\mathbf{C}) \mid g J^{t} g=J\right\} \text {, } \\
& d(a)=\left(\begin{array}{cc}
a & \\
& K^{t} a^{-1} K
\end{array}\right) \text { for } a \in G L_{n}(\mathbf{C}), d\left(t_{1}, \cdots, t_{n}\right)=d\left(\operatorname{diag}\left(t_{1}, \cdots, t_{n}\right)\right), \\
& S=\left\{b \in M_{n}(\mathbf{C}) \mid b={ }^{t} b\right\}, \quad u(b)=\left(\begin{array}{cc}
1 & b K \\
& 1
\end{array}\right) \text { for } b \in S, \\
& U^{\prime}=\left\{\left(x_{i j}\right) \in G L_{n}(\mathbf{C}) \mid x_{i i}=1 \quad \text { and } \quad x_{i j}=0(i>j)\right\}, \\
& U=\left\{d(a) u(b) \mid a \in U^{\prime}, b \in S\right\}, \quad U_{-}=\left\{{ }^{t} u \mid u \in U\right\}, \\
& T=\left\{d\left(t_{1}, \cdots, t_{n}\right) \mid t_{i} \in \mathbf{C}^{\times}\right\}, \quad B=U T, \quad B_{-}=\left\{{ }^{t} b \mid b \in B\right\} .
\end{aligned}
$$

Then the semi-invariant $f_{i}^{\prime}$ corresponding to the fundamental weight $\varpi_{i}$ is given by $f_{i}^{\prime}(x)=\operatorname{det}\left(x_{p q}\right)_{1 \leq p, q \leq i}$ for $x=\left(x_{p q}\right) \in G$. Let us consider the $b$-function $b_{1}(s)$ of $f_{1}^{\prime}$. Since every $\left(B_{-}, B\right)$-double coset contains $J$ in its closure, it is enough to consider the $b$-function in a neighbourhood $U_{-} \cdot T U J$ of $J$. A direct calculation shows that $f_{1}\left(v \cdot d\left(t_{1}, \cdots, t_{n}\right) d(a) u(-b) J\right)=t_{1} f_{1}(d(a) u(-b) J)=t_{1}\left(b_{11}+\right.$ $\left.a_{12} b_{21}+\cdots+a_{1 n} b_{n 1}\right)$ for $v \in U_{-}, t_{p} \in \mathbf{C}^{\times}, a \in\left(a_{p q}\right) \in U^{\prime}$ and $b=\left(b_{p q}\right) \in S$. Hence Conjecture $A$ holds with

$$
b_{1}(s)=s+1 .
$$

Now the verification of Conjectures $B$ and $C$ in this case is easy.

4.6. $\left(G_{2}, 1\right) \quad \underset{\alpha_{1}}{\bigcirc} \notin$

Let $g$ be the totality of the matrices

$$
\left(\begin{array}{ccccccc}
t_{1}+t_{2} & -a_{1} & a_{2} & a_{3} & -a_{4}^{\prime} & -a_{5}^{\prime} & 0 \\
-b_{1} & t_{1} & a_{1}^{\prime} & a_{2} & a_{3} & 0 & a_{5}^{\prime} \\
b_{2} & b_{1}^{\prime} & t_{2} & a_{1} & 0 & -a_{3} & a_{4}^{\prime} \\
2 b_{3} & 2 b_{2} & 2 b_{1} & 0 & 2 a_{1} & 2 a_{2} & 2 a_{3} \\
-b_{4}^{\prime} & b_{3} & 0 & b_{1} & -t_{2} & -a_{1}^{\prime} & -a_{2} \\
-b_{5}^{\prime} & 0 & -b_{3} & b_{2} & -b_{1}^{\prime} & -t_{1} & a_{1} \\
0 & b_{5}^{\prime} & b_{4}^{\prime} & b_{3} & -b_{2} & b_{1} & -t_{1}-t_{2}
\end{array}\right)
$$


and $G$ the group generated by $\{\exp X \mid X \in \mathfrak{g}\}$, where $\exp X=\sum_{n \geq 0} X^{n} / n !$. Then $\mathrm{g}$ is the isotropy subalgebra of $\mathrm{gl}_{7}$ (acting on $\wedge^{3} \mathbf{C}^{7}$ ) at $v_{0}=-u_{1} \wedge u_{5} \wedge u_{6}$ $+u_{2} \wedge u_{3} \wedge u_{7}+u_{1} \wedge u_{4} \wedge u_{7}-u_{2} \wedge u_{4} \wedge u_{6}-u_{3} \wedge u_{4} \wedge u_{5}$ and is a simple Lie algebra of type $G_{2}$. The isotropy subgroup of $G L_{7}$ at $v_{0}$ consists of 3 components $G, G \omega$ and $G \omega^{2}$, where $\omega$ is a scalar matrix of order 3. Since

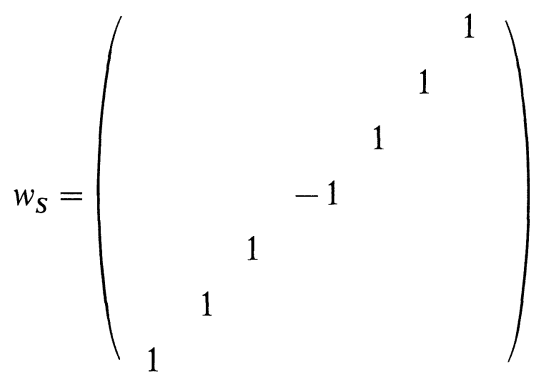

fixes $v_{0}$, it belongs to $G$. (Compare the determinant.) Let $B$ (resp. $B_{-}, T$ ) be the totality of upper triangular (resp. lower triangular, diagonal) matrices in $G$. Then $B$ and $B_{-}$are opposed Borel subgroups and $T$ is the maximal torus. The above element $w_{s}$ represents the longest element of the Weyl group. Let $\mathrm{t}=\operatorname{Lie}(T)$ and $\lambda_{i}$ be its character defined by diag $\left(t_{1}+t_{2}, t_{1}, t_{2}, 0\right.$, $\left.-t_{2},-t_{1},-t_{1}-t_{2}\right) \rightarrow t_{i}$. The simple roots are $\alpha_{1}=\lambda_{2}$ and $\alpha_{2}=\lambda_{1}-\lambda_{2}$, the Dynkin diagram is given by $\alpha_{1} \Leftarrow \alpha_{2}$, and the fundamental weights are $\varpi_{1}=\lambda_{1}+\lambda_{2}$ and $\varpi_{2}=2 \lambda_{1}+\lambda_{2}$. Let $\Delta_{i}(x)=\operatorname{det}\left(x_{p q}\right)_{1 \leq p . q \leq i}$ for $x=\left(x_{p q}\right) \in G$. Then the semi-invariants corresponding to fundamental weights are given by $f^{\varpi_{1}}=\Delta_{1}$, and $f^{\varpi_{2}}=\Delta_{2}$. (We also have $\Delta_{3}=\Delta_{4}=f^{2 \varpi_{1}}, \Delta_{5}=f^{\varpi_{2}}, \Delta_{6}=f^{\varpi_{1}}$ and $\Delta_{7} \equiv 1$.) Let $U_{s}$ be the totality of the matrices

$$
u_{s}=\left(\begin{array}{ccccccc}
1 & -a_{1} & a_{2} & a_{3} & \frac{1}{2} a_{1} a_{3} & \frac{1}{2} a_{2} a_{3} & a_{3}^{2} \\
& 1 & 0 & a_{2} & a_{3}+a_{1} a_{2} & a_{2}^{2} & \frac{1}{2} a_{2} a_{3} \\
& & 1 & a_{1} & a_{1}^{2} & -a_{3}+a_{1} a_{2} & \frac{1}{2} a_{1} a_{3} \\
& & & 1 & 2 a_{1} & 2 a_{2} & 2 a_{3} \\
& & & & 1 & 0 & -a_{2} \\
& & & & & 1 & a_{1}
\end{array}\right)
$$

and $U_{l}$ the totality of the matrices

$$
u_{l}=\left(\begin{array}{ccccccc}
1 & 0 & 0 & 0 & -a_{4}^{\prime} & -a_{5}^{\prime \prime}+a_{1}^{\prime} a_{4}^{\prime} & 0 \\
& 1 & a_{1}^{\prime} & 0 & 0 & 0 & a_{5}^{\prime \prime} \\
& & 1 & 0 & 0 & 0 & a_{4}^{\prime} \\
& & & 1 & 0 & 0 & 0 \\
& & & & 1 & -a_{1}^{\prime} & 0 \\
& & & & & 1 & 0 \\
& & & & & & 1
\end{array}\right) .
$$


Then the open neighbourhood $B_{-} \cdot U_{s} U_{l} w_{S}$ of $w_{S}$ in $G$ is naturally isomorphic to $B_{-} \times U_{l} \times U_{s}$, and a direct calculation shows that $f^{\varpi_{1}}\left(b^{\prime} u_{s} u_{l} w_{S}\right)=\varpi_{1}\left(b^{\prime}\right)$ $\times\left(-a_{1} a_{5}^{\prime \prime}+a_{2} a_{4}^{\prime}+a_{3}^{2}\right)$ for $b^{\prime} \in B_{-}$. Hence Conjecture $A$ holds for $I=\{2\}$ with the $b$-function

$$
b_{1}(s)=(s+1)\left(s+\frac{5}{2}\right) .
$$

Now the verification of Conjectures $B$ and $C$ in this case is easy.

Remark 4.7. Although we can write down semi-invariants explicitly in many cases, it is difficult to calculate their $b$-functions in an elementary way except for a few extremely simple cases such as (4.5) and (4.6). Thus we need an algorithm to calculate them, which we shall give in the next section. Without to say, once the $b$-functions are calculated explicitly for a specific $I$, the verification of Conjectures $B$ and $C$ for this specific case is not difficult, and actually we can do in every example below. Unfortunately, our algorithm to calculate the $b$-functions verifies only one half of Conjecture $A$, namely, the existence of the micro-differential operators $Q_{\mu}(\lambda)$. The author hopes to discuss the differential operators $P_{\mu}$ in a different place.

\section{§5. Holonomy Diagrams}

5.0. In this section, we give a modification of the techniques developped in [24], suitably for the calculation of $b$-functions of semi-invariants. The main difficulty of the modification lies in finding codimension one intersections of irreducible components of $\mathbf{W}_{0}\left(f^{\delta}\right)$ (the characteristic variety of $\mathscr{N}(\lambda)$ ) and in showing the local irreducibility of components. Our techniques to find codimension one intersections are given in (5.8)-(5.13). We discuss the local irreducibility in (5.16) and (5.17).

5.1. Let $L_{g}: G \rightarrow G$ (resp. $R_{g}: G \rightarrow G$ ) be the left (resp. right) translation, i.e., $L_{g}(x)=g x$ (resp. $R_{g}(x)=x g$ ). For a tangent vector $X \in T_{x} G$ at $x \in G$ and for $g \in G$, we write $g X$ (resp. $X g$ ) for $\left(L_{g}\right)_{*} X$ (resp. $\left.\left(R_{g}\right)_{*} X\right)$. Then these "products" are associative, i.e., $g_{1}\left(g_{2} X\right)=\left(g_{1} g_{2}\right) X,\left(g_{1} X\right) g_{2}=g_{1}\left(X g_{2}\right)$ and $\left(X g_{1}\right) g_{2}=X\left(g_{1} g_{2}\right)$. Let $T_{e} G=\mathfrak{g}$. Then the tangent bundle $T G$ of $G$ is the totality of the "products" $g X(g \in G, X \in \mathfrak{g})$. Note also that $g X g^{-1}=(\operatorname{ad} g) X$.

5.2. Let $I$ be a subset of $S, I^{\prime}=w_{S} I w_{S}, \quad P=P(I), \quad P^{\prime}=P\left(I^{\prime}\right)$, and $w \in\left(W_{I^{\prime}} \backslash W_{/}^{\prime} W_{I}\right)_{l}$. Henceforth in this section, we fix $I$. Since $B w B$ is an open neighbourhood of $w$ in $P^{\prime} w P, w^{-1} T_{w}\left(P^{\prime} w P\right)=w^{-1} T_{w}(B w B)=T_{e}\left(w^{-1} B w B\right)=$ $\mathfrak{b}^{w}+\mathfrak{b}=\mathrm{t}+\sum_{\alpha \in w^{-1} R_{+}} \mathfrak{g}(\alpha)+\sum_{\alpha \in R_{+}} \mathfrak{g}(\alpha)$. (We write $x^{y}=y^{-1} x y$ and ${ }^{y} x=y x y^{-1}$.) Put $T\left(P^{\prime} w P\right)_{0}^{\perp}:=\bigcup_{g \in P^{\prime} w P}\left\{\xi \in T_{g}^{*} G \mid \xi \perp T_{g}\left(P^{\prime} w P\right)\right\}$, and let $T\left(\mathrm{P}^{\prime} w P\right)^{\perp}$ be its Zariski closure in $T^{*} G$, which is called the conormal bundle. Identifying $g$ 
with its dual by the Killing form, we may consider $T\left(P^{\prime} w P\right)^{\perp}$ as a subvariety of $T G$. Then

$$
w^{-1} T_{w}\left(P^{\prime} w P\right)^{\perp}=\sum_{\alpha \in w^{-1} R_{+} \cap R_{+}} g(\alpha) .
$$

Consider the $P^{\prime} \times P$-action defined by $\left(p^{\prime}, p\right) x=p^{\prime} x p^{-1}$ for $p^{\prime} \in P^{\prime}, p \in P$ and $x \in P^{\prime} w P$. The isotropy group $\left(P^{\prime} \times P\right)_{w}$ at $w$ can be identified with $P^{\prime w} \cap P$ by $P^{\prime w} \cap P \ni p \rightarrow\left({ }^{w} p, p\right) \in\left(P^{\prime} \times P\right)_{w}$. For $w X \in T_{w}\left(P^{\prime} w P\right)^{\perp}$ and $p \in P^{\prime w} \cap P$, we have $w\left(p X p^{-1}\right)={ }^{w} p \cdot w X \cdot p^{-1}$. Hence the natural action of $\left(P^{\prime} \times P\right)_{w}$ on $T_{w}\left(P^{\prime} w P\right)^{\perp}$ is identified with the adjoint action of $P^{\prime w} \cap P$ on $\sum_{\alpha \in w^{-1} R_{+} \cap R_{+}} g(\alpha)$. We have $\mathfrak{p}^{\prime w} \cap \mathfrak{p}=\mathrm{t}+\sum_{\alpha \in w^{-1}\left(R_{+} \cup R_{I^{\prime}}\right) \cap\left(R_{+} \cup R_{I}\right)} \mathfrak{g}(\alpha)$. Since $w \in\left(W_{I^{\prime}} \backslash W / W_{I}\right)_{l}, w^{-1} R_{I^{\prime},-} \subset R_{+}$ and $w R_{I,-} \subset R_{+}$. Hence $w^{-1}\left(R_{+} \cup R_{I^{\prime}}\right) \cap\left(R_{+} \cup R_{I}\right)=\left(w^{-1} R_{+} \cap R_{+}\right) \cup w^{-1} R_{I^{\prime},-}$ $\cup R_{I,-}$, where the right hand side is a disjoint union.

5.3. Colocalization Given $w \in\left(W_{I^{\prime}} \backslash W / W_{I}\right)_{l}$. Put $R(w)=w^{-1} R_{+} \cap R_{+}$, $R^{\prime}(w)=w^{-1} R_{I^{\prime},-} \cup R_{I,-}, V^{*}(w)=\sum_{\alpha \in R(w)} \mathfrak{g}(\alpha)$ (cf. (5.2.1)), $G(w)=P^{\prime w^{\prime}} \cap P, \mathfrak{g}(w)=$ Lie $(G(w))\left(=\mathrm{t}+\sum_{\alpha \in R(w) \cup R^{\prime}(w)} \mathrm{g}(\alpha)\right)$ and $\Lambda(w)=T(B w B)^{\perp}=T\left(P^{\prime} w P\right)^{\perp}$. Then the colocalization $[24,4.4]$ of the $P^{\prime} \times P$-action on $G$ at $w$ can be identified with $\left(G(w)\right.$, ad, $\left.V^{*}(w)\right)$ and we have $\mathrm{g}(w)=\mathrm{t}+\sum_{\alpha \in R(w) \cup R^{\prime}\left(w^{\prime}\right)} \mathrm{g}(\alpha)$.

5.4. Good Lagrangian Recall that $\delta=\sum_{i \in S-I} \varpi_{i}, \mathcal{N}=\mathscr{D}_{\mathrm{G}}[s] f^{\lambda+s \delta}, \mathcal{N}^{\wedge}(\lambda)$ $=\mathscr{N} / s \mathscr{N}$, and $f^{\lambda+0 \delta}=\left(f^{\lambda+s \delta} \bmod s \mathscr{N}\right)$ for $\lambda=\sum_{i \in S-I} \lambda_{i} \varpi_{i}$. When we are considering $f^{\lambda+0 \delta}$, we say that $\Lambda(w)\left(w \in\left(W_{I^{\prime}} \backslash W / W_{I}\right)_{l}\right)$ is a good Lagrangian if

$$
\left(G(w), \text { ad, } V^{*}(w)\right) \text { is prehomogeneous, and }
$$

$$
\Lambda(w) \subset \mathrm{Ch} \mathscr{D}_{\mathrm{G}} f^{\lambda+0 \delta}=\mathbf{W}_{0}\left(f^{\delta}\right) .
$$

Then as in $[10,0.4]$, we can show that there exist $A_{0} \in \mathrm{t}$ and an element $Y_{0}$ in the open $G(w)$-orbit of $V^{*}(w)$ such that $\left(\operatorname{ad} A_{0}\right) Y_{0}=Y_{0}$. By $(2.6,(3))$ and by the definition of $\mathbf{W}$ and $\mathbf{W}_{0}$, the condition (5.4.2) is equivalent to $\Lambda(w) \subset$ Ch $\mathscr{D}_{G}[s] f^{\lambda+s \delta}=\mathbf{W}\left(f^{\delta}\right)$. Fix such an element $w$. We know that $\mathcal{N}(\lambda)$ is simple holonomic in a neighbourhood of a generic point of a good Lagrangian $[10,2.8]$. Hence we can consider the principal symbol and the order of $f^{\lambda+0 \delta}$ there. See [24] for the definitions of 'simple holonomic', 'principal symbol' and 'order'.

5.5. Order Here we give an algorithm to calculate the order of $f^{i+0 \delta}$. Assume that $\Lambda(w)\left(w \in\left(W_{I} \backslash W / W_{I}\right)_{l}\right)$ is a good Lagrangian. Take $A_{0} \in \mathfrak{t}$ so that $\left(\operatorname{ad} A_{0}\right) Y_{0}=Y_{0}$. Then $\operatorname{tr}\left(\operatorname{ad} A_{0} \mid V^{*}(w)\right)=\sum_{\alpha \in R(w)}\left\langle\alpha, A_{0}\right\rangle$ and $\operatorname{dim} V^{*}(w)=$ card $R(w)$. Since $f_{i}\left({ }^{w} p x p^{-1}\right)=\left(w_{S} \varpi_{i}\right)\left({ }^{w} p\right) \varpi_{i}\left(p^{-1}\right) f_{i}(x)$ for $p \in G(w)=P^{\prime w^{\prime}} \cap P, f_{i}$ is a relative invariant with respect to $g(w)$, and the value of its character at $A_{0}$ is $\left\langle w^{-1} w_{S} \varpi_{i}-\varpi_{i}, A_{0}\right\rangle$. Hence the order of $f^{\lambda+0 \delta}$ at the conormal bundle $\Lambda(w)=T\left(P^{\prime} w P\right)^{\perp}$ is given by 


$$
\operatorname{ord}_{\Lambda(w)} f^{\lambda+0 \delta}=\left\langle w^{-1} w_{S} \lambda-\lambda, A_{0}\right\rangle-\sum_{\alpha \in R(w)}\left\langle\alpha, A_{0}\right\rangle+\frac{1}{2} \operatorname{card} R(w),
$$

(cf. $[24,4.14]$ and $[10,3.3]$ ).

Remark 5.5.2. If $\left(G(w), V^{*}(w)\right)$ is prehomogenous, we can consider the right hand side of (5.5.1). If it really depends on the choice of $A_{0}$, then $\Lambda(w)$ is not contained in $\mathbf{W}_{0}\left(f^{\delta}\right)=\mathrm{Ch} \mathscr{D}_{G} f^{\lambda+0 \delta}$.

5.6. Intersection exponents Assume that $\Lambda(w)$ is a good Lagrangian, $Y_{1} \in V^{*}(w)$ lies in an orbit of codimension one, and $w Y_{1} \in \Lambda(w)$ belongs to another good Lagrangian variety $\Lambda\left(w^{\prime}\right) . \quad$ Then $\operatorname{dim} \Lambda\left(w^{\prime}\right) \cap \Lambda(w)=\operatorname{dim} \Lambda(w)-1$. Assume that $\Lambda\left(w^{\prime}\right) \cap \Lambda(w)$ is not contained in any irreducible component of $\mathbf{W}_{0}\left(f^{\delta}\right)$ other than $\Lambda(w)$ or $\Lambda\left(w^{\prime}\right)$, and that $\Lambda(w)$ and $\Lambda\left(w^{\prime}\right)$ are locally irreducible at $w Y_{1}$ as analytic spaces. (Cf. (5.17).) Find an element $A_{1} \in \mathfrak{g}(w)$ such that $\left(\operatorname{ad} A_{1}\right) Y_{1}=Y_{1}$. Let $(\mu: v)$ be the intersection exponent of $\Lambda\left(w^{\prime}\right)$ to $\Lambda(w)$ $[24,6.4]$. Let $\tilde{V}=V^{*}(w) /(\operatorname{adg} \mathfrak{g}(w)) Y_{1}$. If the value of $\operatorname{tr}\left(A_{1} \mid \tilde{V}\right)$ is independent of the choice of $A_{1}$, then

$$
\operatorname{tr}\left(A_{1} \mid \tilde{V}\right)=\frac{\mu}{\mu+v} .
$$

Since $\nu$ and $\mu$ are relatively prime, non-negative integers, they are uniquely determined by this formula. If the value depends on $A_{1}$, then $\mu=1$ and $v=0$. (Note that the intersection exponents depend only on the characteristic cycle. Hence it is enough to consider $\mathscr{D} f^{0 \delta}=\mathscr{D}[s]\left(f^{\delta}\right)^{s} / s \mathscr{D}[s]\left(f^{\delta}\right)^{s}$. Since only one function $f^{\delta}$ appears in this $\mathscr{D}$-module, the argument of [24] works and we get the above formula.)

Lemma 5.7. $[24,6.6]$ Let $w, w^{\prime} \in\left(W_{I^{\prime}} \backslash W / W_{I}\right)_{l}, \delta=\sum_{i \in S-I} \varpi_{i}$ and $\mathfrak{g}_{0}(w)=$ $\left\{A \in \mathfrak{g}(w) \mid\left\langle w_{S} \delta,{ }^{w} A\right\rangle-\langle\delta, A\rangle=0\right\}$. Assume that $\Lambda(w) \quad\left(\right.$ or $\left.\Lambda\left(w^{\prime}\right)\right) \subset \mathbf{W}\left(f^{\delta}\right)$, $Y_{2} \in V^{*}(w)$, the codimension of $\left(\operatorname{ad~}_{0}(w)\right) Y_{2}$ in $V^{*}(w)$ is one, and $w Y_{2} \in \Lambda\left(w^{\prime}\right)$. Then $\Lambda(w) \cup \Lambda\left(w^{\prime}\right) \subset \mathbf{W}\left(f^{\delta}\right)$. Moreover, $\mathbf{W}\left(f^{\delta}\right)$ is non-singular in a neighbourhood of $w Y_{2}$.

5.8. Intersection of conormal bundles (1) In the special case wher $w>w^{\prime}$ and $l(w)-l\left(w^{\prime}\right)=1$, we can understand the intersection $\Lambda(w) \cap \Lambda\left(w^{\prime}\right)$ fairly well. First, let us consider this case. Put $U_{\alpha}=x_{\alpha}(\mathbf{C}), U_{\alpha}^{\times}=U_{\alpha}-\{e\}$, $U(z)=\prod_{\alpha>0, z \alpha<0} U_{z \alpha}\left(\subset U_{-}\right)$, for $\alpha \in R$ and $z \in W$. Suppose that

$$
\begin{aligned}
& w=u r_{\beta} v, \quad l(w)=l(u)+1+l(v)=: n, \quad \beta \in \Pi, \\
& w^{\prime}=u v, \quad l\left(w^{\prime}\right)=l(u)+l(v) .
\end{aligned}
$$

Note that $u \beta>0, v^{-1} \beta>0$ and $B z B=B U(z) z$.

Lemma 5.8.1. In a neighbourhood of $\dot{w}^{\prime} \in G$, we have an isomorphism 
$\left(B w^{\prime} B, B w B, G\right) \simeq\left(\mathbf{C}^{n-1} \times\{0\} \times\{0\}^{N-n}, \mathbf{C}^{n-1} \times \mathbf{C}^{\times} \times\{0\}^{N-n}, \mathbf{C}^{n-1} \times \mathbf{C} \times \mathbf{C}^{N-n}\right)$. where $N=\operatorname{dim} G . \quad$ Especially $\Lambda(w)$ and $\Lambda\left(w^{\prime}\right)$ have an intersection of codimension one.

Proof. Since $B u B=u U\left(u^{-1}\right) B$, we have natural isomorphisms

$$
\begin{aligned}
& u U\left(u^{-1}\right) \times B v B \simeq B u v B, \\
& u U\left(u^{-1}\right) \times B r v B \simeq B u r v B \quad\left(r:=r_{\beta}\right), \\
& u U\left(u^{-1}\right) \times B u^{-1} w_{S} B w_{S} u v \simeq B w_{S} B w_{S} u v .
\end{aligned}
$$

Hence it suffices to give an isomorphism

$$
\begin{aligned}
& \left(B v B, B r v B, B U\left(u^{-1} w_{S}\right) v\right)=\left(B U(v) v, B U(r v) r v, B U\left(u^{-1} w_{S}\right) v\right) \\
& \simeq\left(\mathbf{C}^{m-1} \times\{0\} \times\{0\}^{M-m}, \mathbf{C}^{m-1} \times \mathbf{C}^{\times} \times\{0\}^{M-m}, \mathbf{C}^{m-1} \times \mathbf{C} \times \mathbf{C}^{M-m}\right)
\end{aligned}
$$

in a neighbourhood of $v$ in $B U\left(u^{-1} w_{S}\right) v$, where $m=\operatorname{dim} B U(v) v+1$ and $M=\operatorname{dim} B U\left(u^{-1} w_{S}\right) v . \quad$ It is easy to see that $U(v) \subset U\left(u^{-1} w_{S}\right)$, i.e.,

$$
B U(v) \cap B U\left(u^{-1} w_{S}\right)=B U(v) .
$$

Hence, in order to prove (5.8.2), it is enough to show that

$$
B U(r v) r \cap B U\left(u^{-1} w_{S}\right)=B U_{-\beta}^{\times} U(v) .
$$

Since $B U(r v) r v=B r v B=B r B v B=B r B U(v) v=B r U_{\beta} U(v) v$,

$$
B U(r v) r=B r U_{\beta} U(v) .
$$

If $t \neq 0, r_{\beta} \cdot x_{\beta}(t) \in T x_{\beta}(-t) x_{-\beta}\left(t^{-1}\right) x_{\beta}(-t) \cdot x_{\beta}(t) \in B x_{-\beta}\left(t^{-1}\right)$. Hence

$$
B r U_{\beta}^{\times}=B U_{-\beta}^{\times} .
$$

By (5.8.5) and (5.8.6), $B U(r v) r=B r U(v) \cup B U_{-\beta}^{\times} U(v)$. Hence in order to prove (5.8.4), it suffices to show that

$$
\begin{aligned}
& B U_{-\beta}^{\times} U(v) \subset B U\left(u^{-1} w_{S}\right), \quad \text { and } \\
& B r U(v) \cap B U\left(u^{-1} w_{S}\right)=\phi .
\end{aligned}
$$

It is easy to see that $U_{-\beta} \cup U(v) \subset U\left(u^{-1} w_{S}\right)$. Hence we get (5.8.7), and (5.8.8) reduces to $r \notin B U\left(u^{-1} w_{S}\right)$, i.e., $r u^{-1} w_{S} \notin B U\left(u^{-1} w_{S}\right) u^{-1} w_{S}=B u^{-1} w_{S} B$.

Lemma 5.8.9. Let $w$ and $w^{\prime}$ be as above. Then

$$
w^{w^{-1}}\left(T_{w^{\prime}}\left(B w^{\prime} B\right)^{\perp} \cap T_{w^{\prime}}(B w B)^{\perp}\right)=\sum_{\alpha \in E} \mathfrak{g}(\alpha)
$$

where $E=\left\{x \in R_{+} \mid v \alpha>0, u v \alpha>0, v \alpha \neq \beta\right\}$. 
Proof. The assertion follows from the equalities

$$
\begin{aligned}
(u v)^{-1} B u v B & =v^{-1} U\left(u^{-1}\right) v \cdot v^{-1} B v \cdot v^{-1} U(v) v \\
& =\prod_{\substack{v \alpha>0 \\
u v \alpha<0}} U_{-\alpha} \cdot \prod_{v \alpha<0} U_{-\alpha} \cdot T \cdot \prod_{\substack{\alpha<0 \\
v \alpha>0}} U_{-\alpha}
\end{aligned}
$$

and

$$
\begin{aligned}
(u v)^{-1} B u r v B & =(u v)^{-1} B u B \cdot B r v B=(u v)^{-1} u U\left(u^{-1}\right) B \cdot B U(r v) r v \\
& =v^{-1} U\left(u^{-1}\right) B \cdot B U_{-\beta}^{\times} U(v) v \\
& =v^{-1} U\left(u^{-1}\right) v \cdot v^{-1} B v \cdot v^{-1} U_{-\beta}^{\times} v \cdot v^{-1} U(v) v \\
& =\prod_{\substack{v \alpha>0 \\
u v \alpha<0}} U_{-\alpha} \cdot \prod_{v \alpha<0} U_{-\alpha} \cdot T \cdot U_{-v^{-1} \beta}^{\times} \cdot \prod_{\substack{\alpha<0 \\
v \alpha>0}} U_{-\alpha} .
\end{aligned}
$$

Here the third equality holds only in a neighbourhood of $w^{\prime}=u v$. Cf. (5.8.4).

5.9. Next, let us consider a way to find elements in the intersection $\Lambda(w) \cap \Lambda\left(w^{\prime}\right)$ for general $w, w^{\prime} \in\left(W_{I^{\prime}} \backslash W / W_{I}\right)_{l}$. Here we constantly use the notation in (5.1).

First, let us consider the case where

$$
\begin{aligned}
& w=u r v, \quad r=r_{\beta}(\beta \in \Pi), \quad l(w)=l(u)+1+l(v), \\
& w^{\prime}=u v, \quad \text { and } \quad l\left(w^{\prime}\right)=l(u)+l(v),
\end{aligned}
$$

as in (5.8). Take representatives of $u$ and $v$ in $N_{G}(T)$, which we shall denote by the same letters $u$ and $v$, so that $u x_{\beta}(t) u^{-1}=x_{u \beta}(t)$ and $v^{-1} x_{\beta}(t) v=x_{v^{-1} \beta}(t)$. Here $x_{\beta}(t)$ denotes a one parameter subgroup of $G$ such that $\dot{x}_{\beta}(0)=X_{\beta}(=\operatorname{root}$ vector). Put $w_{\beta}(t)=x_{\beta}\left(t^{-1}\right) x_{-\beta}(-t) x_{\beta}\left(t^{-1}\right)\left(t \in \mathbf{C}^{\times}\right)$. Then $w_{\beta}(t)$ represents the element $r \in W$. We take $w_{\beta}(1)$ as a representative element of $r$, and denote $w_{\beta}(1)$ by $r$. Put $g(t)=u x_{\beta}(t) x_{-\beta}(-t) x_{\beta}(t) v$. Then $g(1)=u r v=: w, g(0)=u v=$ : $w^{\prime}$, and

$$
\begin{aligned}
g(t) & =u x_{\beta}\left(t-t^{-1}\right) w_{\beta}(t) x_{\beta}\left(t-t^{-1}\right) v \\
& =x_{u \beta}\left(t-t^{-1}\right) u w_{\beta}(t) v x_{v^{-1} \beta}\left(t-t^{-1}\right) \in B w x_{v^{-1} \beta}\left(t-t^{-1}\right) \subset B w B
\end{aligned}
$$

for $t \neq 0$. Hence if $t \neq 0$, then

$$
\begin{aligned}
& g(t)^{-1} T_{g(t)}(B w B)=T_{e}\left(g(t)^{-1} B g(t) B\right)=\mathfrak{b}^{g(t)}+\mathfrak{b}=\left(\mathfrak{b}^{w}+\mathfrak{b}\right)^{x_{\gamma}\left(t-t^{-1}\right)}, \text { and } \\
& g(t)^{-1} T_{g(t)}(B w B)^{\perp}=V^{*}(w)^{x_{\gamma}\left(t-t^{-1}\right)},
\end{aligned}
$$

where $\gamma=v^{-1} \beta$. (Cf. (5.3) for $V^{*}(w)$.) Hence

$$
w^{\prime-1} T_{w^{\prime}}(B w B)^{\perp} \supset \lim _{t \rightarrow 0} g(t)^{-1} T_{g(t)}(B w B)^{\perp}=\lim _{t \rightarrow \infty} V^{*}(w)^{x_{\gamma}(t)} .
$$


Here, for instance, the most right hand side denotes the totality of $\lim _{t \rightarrow \propto} v(t)^{x_{\gamma}(t)}$. where $v(t)$ is any path in $V^{*}(w)$ such that $\lim v(t)^{x_{\gamma}(t)}$ exists. If $\alpha, \alpha^{\prime}, \alpha+\alpha^{\prime} \in R$, then it is known that $\left[X_{\alpha}, X_{\alpha^{\prime}}\right]=N_{\alpha, \alpha^{\prime}} X_{\alpha+\alpha^{\prime}}$ with some $N_{\alpha . \alpha^{\prime}} \in \mathbf{C}^{\times}$. Take $\alpha \in w^{-1} R_{+} \cap R_{+}$, and let $p=p(\alpha)$ (resp. $q=q(\alpha)$ ) be the integer such that

$$
\alpha, \alpha+\gamma, \cdots, \alpha+p \gamma \in R \text { and }(p+1) \gamma \notin R .
$$

(resp. $\alpha, \alpha+\gamma, \cdots, \alpha+q \gamma \in w^{-1} R_{+} \cap R_{+} \quad$ and $\quad \alpha+(q+1) \gamma \notin w^{-1} R_{+} \cap R_{+}$).

Then

$$
\begin{aligned}
& t^{j} N_{\alpha, \gamma} \cdots N_{\alpha+(j-1) \gamma, \gamma} X_{\alpha+j \gamma}^{x_{\gamma}(t)} \\
& \quad=t^{j} N_{\alpha, \gamma} \cdots N_{\alpha+(j-1) \gamma, \gamma} \sum_{i=0}^{p-j} \frac{t^{i}}{i !} N_{\alpha+j \gamma, \gamma} \cdots N_{\alpha+(j+i-1) \gamma, \gamma} X_{\alpha+(j+i) \gamma} \\
& \quad=\sum_{i=0}^{p} \frac{t^{i}}{(i-j) !} N_{\alpha, \gamma} \cdots N_{\alpha+(i-1) \gamma, \gamma} X_{\alpha+i \gamma} \in V^{*}(w)^{x_{\gamma}(t)}
\end{aligned}
$$

for $0 \leq j \leq q$. Here we understand $\frac{1}{n !}=0$ for $n \in \mathbf{Z}_{<0}$. Note that

$$
\begin{aligned}
& \left|\begin{array}{cccc}
\frac{1}{p !} & \frac{1}{(p-1) !} & \cdots & \frac{1}{(p-q) !} \\
\frac{1}{(p-1) !} & \frac{1}{(p-2) !} & \cdots & \frac{1}{(p-q-1) !} \\
& \ldots \ldots \ldots \ldots \ldots \ldots \ldots \ldots \ldots \ldots
\end{array}\right|
\end{aligned}
$$

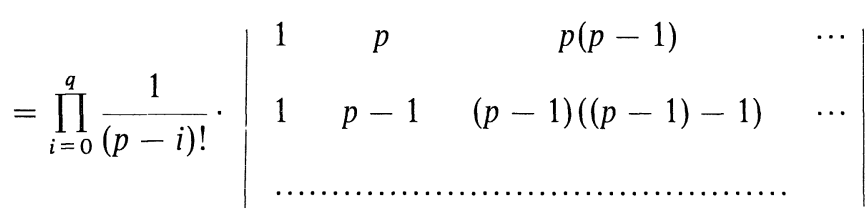

$$
\begin{aligned}
& =\prod_{i=0}^{q} \frac{1}{(p-i) !} \cdot\left|\begin{array}{cccc}
1 & p & p^{2} & \cdots \\
1 & p-1 & (p-1)^{2} & \cdots \\
\ldots \ldots \ldots \ldots \ldots \ldots \ldots
\end{array}\right| \neq 0 .
\end{aligned}
$$

Hence by (5.9.3), we can find elements in $V^{*}(w)^{x_{\gamma}(t)}$ of the form $t^{i} X_{\alpha+i^{\prime}}+$ (terms of lower degree in $t)$ for $p-q \leq i \leq p$. In other words $X_{\alpha+i \gamma}+O\left(t^{-1}\right) \in$ $V^{*}(w)^{x_{\gamma}(t)}(t \rightarrow \infty)$ for $p-q \leq i \leq p$. Hence

$$
V^{\prime}:=\sum_{\substack{\alpha \in w^{-1} R_{+} R_{+} \\ p(\alpha)-q(\alpha) \leq j \leq p(\alpha)}} \mathfrak{g}(\alpha+j \gamma) \subset \lim _{t \rightarrow \infty} V^{*}(w)^{x_{\gamma}(t)} .
$$

5.9.5. In general, for a $\mathbf{C}$-vector space $V$, denote by $\operatorname{Grass}_{m}(V)$ the totality of $m$-dimensional linear subspaces of $V$, with the natural structure of an algebraic 
variety. For a smooth algebraic variety $X$ over $\mathbf{C}$, let $T^{*} X$ be the cotangent bundle and $\operatorname{Grass}_{m}\left(T^{*} X\right)=\bigcup_{x \in X} \operatorname{Grass}_{m}\left(T_{x}^{*} X\right)$ (disjoint union) with the natural structure of an algebraic variety. We denote the limit in $\operatorname{Grass}_{m}(V)$ or Grass $_{m}\left(T^{*} X\right.$ ) (with the classical topology) by $G$-lim, and the limit in $T^{*} X$ by lim. Let $\pi$ (resp. $\pi_{m}$ ) be the projection $T^{*} X \rightarrow X$ (resp. $\operatorname{Grass}_{m}\left(T^{*} X\right) \rightarrow X$ ). For a smooth irreducible subvariety $Y$ of $X$ (not necessarily closed), let $\operatorname{Grass}_{m}\left((T Y)^{\perp}\right)$ be the Zariski closure in $\operatorname{Grass}_{m}\left(T^{*} X\right)$ of the (disjoint) union $\bigcup_{y \in Y} \operatorname{Grass}_{m}\left(\left(T_{y} Y\right)^{\perp}\right)$. Note that it is also the closure with respect to the classical topology.

Lemma 5.9.6. If $U \in \operatorname{Grass}_{m}\left((T Y)^{\perp}\right)$, then $U \subset(T Y)^{\perp}$.

Proof. Let $\Xi$ be the subvariety of $\operatorname{Grass}_{m}\left(T^{*} X\right) \times{ }_{X} T^{*} X$ consisting of pairs $(U, u)$ such that $U \ni u$. Let $\operatorname{Grass}_{m}\left(T^{*} X\right) \stackrel{\alpha}{\leftarrow} \Xi \stackrel{\beta}{\rightarrow} T^{*} X$ be the projections. Note that $\operatorname{Grass}_{m}\left((T Y)^{\perp}\right)$ is irreducible, $\alpha$ is a vector bundle, and $\beta$ is a projective morphism. Hence $Z:=\beta \alpha^{-1} \operatorname{Grass}_{m}\left((T Y)^{\perp}\right)$ is a closed irreducible subvariety of $T^{*} X$. Since $\pi(Z) \subset \bar{Y}$ and $Z \cap \pi^{-1}(Y)=(T Y)^{\perp} \cap \pi^{-1}(Y), Z=$ $(T Y)^{\perp}$. This implies the asserion.

Now we return to the cotangent bundle of $G$. Let $v$ be an element of the most right hand side of (5.9.4), and $v(t)$ a path in $V^{*}(w)$ such that $v(t)^{x_{\gamma}(t)} \rightarrow v(t \rightarrow \infty)$. Put $m:=\operatorname{dim} V^{*}(w)$. Define $\tau: \mathbf{C} \rightarrow \operatorname{Grass}_{m}(\mathfrak{g})$ by $\tau(t)=$ $V^{*}(w)^{x_{\gamma}(t)}$. Considering the normalization of the Zariski closure of $\tau(\mathbb{C})$, we can show that the boundary of $\tau(\mathbf{C})$, or $\tau(\mathbf{C})$ itself, consists of only one point. Hence $G-\lim _{t \rightarrow \infty} V^{*}(w)^{x_{\gamma}(t)}$ exists, which we shall denote by $\tilde{V}^{\prime}$. Then $\left(v(t)^{x_{\gamma}(t)}, V^{*}(w)^{x_{\gamma}(t)}\right)$ is a path lying in $\left\{(x, V) \in \mathfrak{g} \times \operatorname{Grass}_{m}(\mathfrak{g}) \mid x \in V\right\}$. Hence its limit point $\left(v, \tilde{V}^{\prime}\right)$ also lies in the same set, i.e., $v \in \tilde{V}^{\prime}$. Thus

$$
\lim _{t \rightarrow \infty} V^{*}(w)^{x_{\gamma}(t)} \subset \tilde{V}^{\prime} .
$$

Put $E_{0}=w^{-1} R_{+} \cap R_{+}$and $E_{1}=\left\{\alpha+j \gamma \mid \alpha \in E_{0}, p(\alpha)-q(\alpha) \leq j \leq p(\alpha)\right\}$. For each $\alpha, \beta \in R$, it is well known that $\{j \in \mathbb{Z} \mid \alpha+j \beta \in R\}$ is an interval. Hence

$$
E_{0}=\bigcup_{\alpha \in A}\{\alpha+j \gamma \mid 0 \leq j \leq q(\alpha)\} \quad \text { (disjoint union) }
$$

with some subset $A$ of $E_{0}$, and

$$
E_{1}=\bigcup_{\alpha \in A}\{\alpha+j \gamma \mid p(\alpha)-q(\alpha) \leq j \leq p(\alpha)\} \quad \text { (disjoint union). }
$$

Hence

$$
\operatorname{dim} \tilde{V}^{\prime}=m=\operatorname{dim} V^{*}(w)=\operatorname{card} E_{0}=\operatorname{card} E_{1}=\operatorname{dim} V^{\prime} .
$$

By (5.9.4), (5.9.7) and (5.9.8), $V^{\prime}=G-\lim _{t \rightarrow \infty} V^{*}(w)^{x_{\gamma}(t)}$. Hence 


$$
w^{\prime} V^{\prime}=G-\lim _{t \rightarrow 0} g(t) V^{*}(w)^{x_{\gamma}\left(t-t^{-1}\right)}=G-\lim _{t \rightarrow 0} T_{g(t)}(B w B)^{\perp} \in \operatorname{Grass}_{m}\left(T(B w B)^{\perp}\right) .
$$

On the other hand

$$
w^{\prime} V^{\prime}=G-\lim _{t \rightarrow 0} T_{g(t)}(B w B)^{\perp} \in \operatorname{Grass}_{m}\left(T\left(B w^{\prime} B\right)^{\perp}\right),
$$

because $\{B z B \mid z \in W\}$ is a Whitney stratification of $G$. (By [30], $\{B z B \mid z \in W\}$ has a refinement which satisfies the Whitney condition. Taking into account the $B \times B$-action, we can show that $\{B z B \mid z \in W\}$ itself is a Whitney stratification.)

Next assume that

$$
\begin{aligned}
& w^{\prime}=u^{\prime} r^{\prime} v^{\prime}, \quad r^{\prime}=r_{\beta^{\prime}}\left(\beta^{\prime} \in \Pi\right), \quad l\left(w^{\prime}\right)=l\left(u^{\prime}\right)+1+l\left(v^{\prime}\right), \\
& w^{\prime \prime}=u^{\prime} v^{\prime}, \quad \text { and } \quad l\left(w^{\prime \prime}\right)=l\left(u^{\prime}\right)+l\left(v^{\prime}\right) .
\end{aligned}
$$

Put $g^{\prime}(t)=u^{\prime} x_{\beta^{\prime}}(t) x_{-\beta^{\prime}}(-t) x_{\beta^{\prime}}(t) v^{\prime}$ and $\gamma^{\prime}=v^{\prime-1} \beta^{\prime}(>0)$. As in the first step, $g^{\prime}(t)$ can be expressed as $b^{\prime}(t) w^{\prime} x_{\gamma^{\prime}}\left(t-t^{-1}\right)$ with some $b^{\prime}(t) \in B$ for $t \in \mathbf{C}^{\times}$. Then

$$
g^{\prime}(t) V^{\prime x_{\gamma^{\prime}}\left(t-t^{-1}\right)}=b^{\prime}(t) w^{\prime} V^{\prime} x_{\gamma^{\prime}}\left(t-t^{-1}\right) \in \operatorname{Grass}_{m}\left(T(B w B)^{\perp}\right) \cap \operatorname{Grass}_{m}\left(T\left(B w^{\prime} B\right)^{\perp}\right) .
$$

As in the first step, we can show that $G$-lim of the left hand exists, which we shall denote by $w^{\prime \prime} V^{\prime \prime}$. In the same way, we can determine the explicit form of $V^{\prime \prime}$, and can show that

$$
w^{\prime \prime} V^{\prime \prime} \in \operatorname{Grass}_{m}\left(T(B w B)^{\perp}\right) \cap \operatorname{Grass}_{m}\left(T\left(B w^{\prime} B\right)^{\perp}\right) \cap \operatorname{Grass}_{m}\left(T\left(B w^{\prime \prime} B\right)^{\perp}\right) .
$$

Repeating such an argument and using (5.9.6), we get the following algorithm.

5.10. Intersection of conormal bundles (2) Let $w, w^{\prime} \in\left(W_{I^{\prime}} \backslash W / W_{I}\right)_{l}$ and suppose that there exists a sequence $w=w_{0}, w_{1}, \cdots, w_{n}=w^{\prime}$ of elements in $W$ such that

$$
\begin{aligned}
& w_{i}=u_{i} r_{\beta_{1}} v_{i} \quad\left(\beta_{i} \in \Pi\right), \quad l\left(w_{i}\right)=l\left(u_{i}\right)+1+l\left(v_{i}\right), \\
& w_{i+1}=u_{i} v_{i}, \quad \text { and } \quad l\left(w_{i+1}\right)=l\left(u_{i}\right)+l\left(v_{i}\right)
\end{aligned}
$$

for $0 \leq i<n$. Note that $w=w_{0}>w_{1}>\cdots>w_{n}=w^{\prime}$. Put $\gamma_{i}=v_{i}^{-1}\left(\beta_{i}\right)$ $(0 \leq i<n)$ and define subset $E_{i}(0 \leq i \leq n)$ of $R_{+}$as follows. (Note that $\gamma_{i}>0$.) First we put

$$
E_{0}=w_{0}^{-1} R_{+} \cap R_{+} .
$$

We construct $E_{i+1}$ from $E_{i}$ as follows. For an element $\alpha \in E_{i}$, let $p_{i}=p_{i}(\alpha)$ and $q_{i}=q_{i}(\alpha)$ be the integers such that

$$
\begin{array}{ll}
\alpha, \alpha+\gamma_{i}, \cdots, \alpha+p_{i} \gamma_{i} \in R, & \alpha+\left(p_{i}+1\right) \gamma_{i} \notin R, \\
\alpha, \alpha+\gamma_{i}, \cdots, \alpha+q_{i} \gamma_{i} \in E_{i}, & \alpha+\left(q_{i}+1\right) \gamma_{i} \notin E_{i} .
\end{array}
$$

Define $E_{i+1}$ by 


$$
E_{i+1}=\left\{\alpha+j \gamma_{i} \mid \alpha \in E_{i}, p_{i}(\alpha)-q_{i}(\alpha) \leq j \leq p_{i}(\alpha)\right\} .
$$

Put $V_{i}=\sum_{\alpha \in E_{1}} \mathfrak{g}(\alpha)$. Then

$$
\begin{aligned}
& w^{\prime} V_{n} \in \operatorname{Grass}_{m}\left(T(B w B)^{\perp}\right) \cap \operatorname{Grass}_{m}\left(T\left(B w^{\prime} B\right)^{\perp}\right), \text { and } \\
& w^{\prime} V_{n} \subset T(B w B)^{\perp} \cap T\left(B w^{\prime} B\right)^{\perp}
\end{aligned}
$$

where $m=\operatorname{codim}_{G}(B w B)$.

5.11. The algorithm given in (5.10) often fails to be efficient to find codimension one intersections of $\Lambda(w)$ 's. Let us give a technique to make up for this fault.

Lemma 5.11.1. Let notation be as in (5.9.5). If $y$ is a normal point of the Zariski closure of $Y$, then $\operatorname{Grass}_{m}\left((T Y)^{\perp}\right) \cap \pi_{m}^{-1}(y)$ is connected (in the Zariski topology).

Proof. Since $\pi_{m}$ : $\operatorname{Grass}_{m}\left((T Y)^{\perp}\right) \rightarrow Y$ is a birational proper morphism, the connectedness follows from Zariski's connectedness theorem.

\section{Lemma 5.11.2. [5, Corollary 1 in p. 85] $\overline{B w B}$ is a normal variety.}

Remark. The proof of the normality given in [5] contained a gap, but the result is valid. See the reviewer's remark given at the end of MR 87g: 17006.

5.11.3. Let notation be as in (5.10). By (5.11.2), we can apply (5.11.1) for $X=G, Y=B w B$, and $y=w^{\prime}$. Thus $\Sigma:=\operatorname{Grass}_{m}\left(T(B w B)^{\perp}\right) \cap \pi_{m}^{-1}\left(w^{\prime}\right)$ is connected. Since $\{B z B \mid z \in W\}$ is a Whitney stratification, $\Sigma \subset \operatorname{Grass}_{m}\left(T\left(B w^{\prime} B\right)^{\perp}\right)$ $\cap \pi_{m}^{-1}\left(w^{\prime}\right)=w^{\prime} \operatorname{Grass}_{m}\left(V^{*}\left(w^{\prime}\right)\right)$, and $w^{\prime} V_{n} \in \Sigma$ by (5.10.1). Thus we get the following assertion which complements (5.10).

5.12. Intersection of conormal bundles (3) Let $w, w^{\prime} \in\left(W_{I^{\prime}} \backslash W / W_{I}\right)_{l}$ such that $w>w^{\prime}$ and $\left\{V_{i} \mid 0 \leq i \leq n\right\},\left\{V_{i}^{\prime} \mid 0 \leq i \leq n\right\}$ etc. be various sequences of subspaces of $g$ constructed in (5.10) by taking various sequences $\left\{w_{i}\right\}$. Then, there exists a $G\left(w^{\prime}\right)$-stable connected subset of $\operatorname{Grass}_{m}\left(V^{*}\left(w^{\prime}\right)\right)$, say $\Sigma_{0}$, such that $\left\{w^{\prime} V_{n}, w^{\prime} V_{n}^{\prime}, \cdots\right\} \subset w^{\prime} \Sigma_{0} \subset \operatorname{Grass}_{m}\left(T(B w B)^{\perp}\right) \cap \operatorname{Grass}_{m}\left(T\left(B w^{\prime} B\right)^{\perp}\right)$ and $\cup\left(w^{\prime} \Sigma_{0}\right)$ $\subset T(B w B)^{\perp} \cap T\left(B w^{\prime} B\right)^{\perp}$.

5.13. Intersection of conormal bundles (4) If $G$ is of type $A_{l}$, then we may assume that $G=G L_{n}(\mathbf{C})$. (Although we have assumed $G$ to be semisimple, the necessary modification would be obvious.) Since $\left(B \times B, M_{n}(\mathbf{C})\right)$ is a prehomogenous vector space and $G L_{n}(\mathbf{C})$ is an open subset of $M_{n}(\mathbf{C})$, the method given in $[24,6.2]$ can be used.

5.14. Intersection of conormal bundles (5) Besides the techniques to find codimension one intersections of the irreducible components of $\mathbf{W}_{0}$, we also 
need a technique to show that some components do not intersect in codimension one.

Assume that $\Lambda(w) \cap \Lambda\left(w^{\prime}\right)\left(w, w^{\prime} \in W_{0}(I)\right)$ has an irreducible component $\Delta$ such that $\operatorname{dim} \Delta=\operatorname{dim} G-1$. Let $\pi: T^{*} G \rightarrow G$ be the projection and $g \in \pi(\Delta)$ a point such that $\operatorname{dim} \pi^{-1}(g) \cap \Delta=\operatorname{dim} \Delta-\operatorname{dim} \overline{\pi(\Delta)}$. Take $w^{\prime \prime} \in W_{0}(I) \backslash\left\{w, w^{\prime}\right\}$. Since $\Lambda\left(w^{\prime \prime}\right)$ is the Zariski closure of $\bigcup_{b, b^{\prime} \in B} b^{\prime} w^{\prime \prime} V^{*}\left(w^{\prime \prime}\right) b=\bigcup_{b \in B} B w^{\prime \prime} b V^{*}\left(w^{\prime \prime}\right)^{b}$, we have

$$
g^{-1}\left(\pi^{-1}(g) \cap \Lambda\left(w^{\prime \prime}\right)\right) \subset \overline{\bigcup_{b \in B} V^{*}\left(w^{\prime \prime}\right)^{b}}=: M
$$

If $\Lambda\left(w^{\prime \prime}\right)$ contains $\Delta$, then

$$
g^{-1}\left(\pi^{-1}(g) \cap \Delta\right) \subset M
$$

and especially

$$
\operatorname{dim} G-1-\operatorname{dim} \overline{\pi(\Delta)} \leq \operatorname{dim} M
$$

Since $\overline{\pi(\Delta)}$ is a $B \times B$-stable irreducible subset of $G, \overline{\pi(\Delta)}=\overline{B z B}$ for some $z \in W$. Then (5.14.2) can be also expressed as

$$
\text { card } R_{+}-1-l(z) \leq \operatorname{dim} M .
$$

Therefore, if $M$ does not satisfy one of these conditions, then $\Lambda\left(w^{\prime \prime}\right)$ does not contain $\Delta$.

5.15. Besides the algorithm gien in (5.14), the following simple remark is also useful. If $\overline{B w^{\prime \prime} B}$ does not contain $\overline{\pi(\Delta)}=\overline{B z B}$ (i.e., $w^{\prime \prime} \ngtr z$ ), then $\Lambda\left(w^{\prime \prime}\right)$ does not contain $\Delta$.

5.16. In the notation of (5.14), even if $\Delta$ is contained in exactly two Lagrangians $\Lambda(w)$ and $\Lambda\left(w^{\prime}\right)$, it is still possible that the irreducible algebraic variety, say $\Lambda(w)$, would have more than one branches containing $\Delta$. In order to apply the algorithm (5.6) and also (5.20) below, we need to know the local irreducibility of $\Lambda(w)$ at the generic point of $\Delta$.

Assume

(5.16.1) that $w^{\prime}, w \in\left(W_{I^{\prime}} \backslash W / W_{I}\right)_{l}$ and $w^{\prime} \leq w$, that $V^{*}(w)$ has an open dense $G(w)$-orbit and $Y_{0}$ belongs to it, that $w^{\prime-1} \cdot\left(\pi^{-1}\left(w^{\prime}\right) \cap \Delta\right)\left(\subset V^{*}\left(w^{\prime}\right)\right)$ has an open dense $G\left(w^{\prime}\right)$-orbit, say $\Omega$, and $Y_{1}$ belongs to it, and that $Y_{0}$ and $Y_{1}$ belong to the same ad $(G)$-orbit, say $C$, in $\mathrm{g}$.

Then $T(B w B)^{\perp}=T\left(P^{\prime} w P\right)^{\perp}$ (resp. 4$)$ has the open dense $P^{\prime} \times P$-orbit $P^{\prime}\left(w Y_{0}\right) P \quad$ (resp. $\left.P^{\prime}\left(w^{\prime} Y_{1}\right) P\right)$. Let $\Xi=\left\{(g, h, X) \in G \times G \times \mathfrak{g} \mid X \in C \cap \mathfrak{b}^{g} \cap \mathfrak{b}^{h}\right\}$, $\Xi(w)_{0}$ be the subset of $\Xi$ consisting of $(g, h, X)$ such that $g h^{-1} \in B w B, \Xi(w)$ the Zariski closure of $\Xi(w)_{0}$ in $G \times G \times C$, and $p_{i}$ the $i$-th projection of $\Xi$ to 
the $i$-th factor of $G \times G \times \mathfrak{g}$. For $g \in G$ and $X \in \mathfrak{g}, g X \in T(B w B)_{0}^{\perp}$ (cf. (5.2)) if and only if $g \in B w B$ and $X \in \bar{C} \cap \mathfrak{b}^{g} \cap \mathfrak{b}$. (In fact, $g X \in T(B w B)_{0}^{\perp}$ if and only if $g \in B w B$ and $X \in \mathfrak{n}^{g} \cap \mathfrak{n}$, where $\mathfrak{n}=[\mathfrak{b}, \mathfrak{b}]$. If $g=b^{\prime} w b\left(b, b^{\prime} \in B\right)$, then $\mathfrak{n}^{g} \cap \mathfrak{n}=$ $\left(\mathfrak{n}^{w} \cap \mathfrak{n}\right)^{b}=V^{*}(w)^{b}=\left(\right.$ the closure of $\left.C \cap V^{*}(w)^{b}\right)=\bar{C} \cap V^{*}(w)^{b}=\bar{C} \cap \mathfrak{n}^{g} \cap \mathfrak{n}=\bar{C}$ $\cap \mathfrak{b}^{g} \cap \mathfrak{b}$.) Define the closed imbedding $l: T^{*} G \rightarrow G \times G \times g$ by $l(g X)=(g, e, X)$. Then $l\left(T(B w B)_{0}^{\perp}\right)=\left\{(g, e, X) \mid g \in B w B, X \in \bar{C} \cap \mathfrak{b}^{g} \cap b\right\}$. Take an open neighbourhood $O$ of $Y_{1}$ in $g$ which does not intersect $\bar{C} \backslash C$. Then $l\left(T(B w B)_{0}^{\perp}\right)$ $\cap(G \times G \times O)=p_{2}^{-1}(e) \cap \Xi(w)_{0} \cap(G \times G \times O)$, and therefore $l\left(T(B w B)^{\perp}\right)=p_{2}^{-1}(e)$ $\cap \Xi(w)$ in a neighbourhood of $l\left(w^{\prime} Y_{1}\right)$. Hence the irreducibility of the germ of analytic space $\left(T(B w B)^{\perp-} w^{\prime} Y_{1}\right)$ is equivalent to that of $\left(p_{2}^{-1}(e) \cap \Xi(w)\right.$, $\left.l\left(w^{\prime} Y_{1}\right)\right)$. Note that $\Xi(w)=\left\{(g, h, X) \in G \times G \times C \mid g h^{-1} \in \overline{B w B}, \quad X \in \mathfrak{b}^{g} \cap \mathfrak{b}^{h}\right\}$. (Consider the automorphism $(g, h, X) \rightarrow\left(g h^{-1}, h, X\right)$ of $G \times G \times C$, by which $\Xi(w)_{0}$ is mapped to $(B w B \times G \times C) \cap\left\{(x, h, X) \in G \times G \times C \mid X \in\left(\mathfrak{b}^{x} \cap \mathfrak{b}\right)^{h}\right\}$.) Hence $\left(p_{2}^{-1}(e) \cap \Xi(w)\right) \times G \stackrel{\sim}{\rightarrow} \Xi(w)$ by $(g, e, X) \times h \rightarrow\left(g h, h, X^{h}\right)$. Thus the irreducibility of $\left(p_{2}^{-1}(e) \cap \Xi(w),\left(w^{\prime}, e, Y_{1}\right)\right)$ is equivalent to that of $\left(\Xi(w),\left(w^{\prime}, e, Y_{1}\right)\right)$. Put $\Xi\left(w, Y_{1}\right)=\left\{(g, h) \in G \times G \mid g h^{-1} \in \overline{B w B}, Y_{1} \in \mathfrak{b}^{g} \cap \mathfrak{b}^{h}\right\}$. Note that $p_{3}: \Xi(w) \rightarrow C$ is an (analytic) fibre bundle whose fibres are isomorphic to $\Xi\left(w, Y_{1}\right)$. Hence the irreducibility of $\left(\Xi(w),\left(w^{\prime}, e, Y_{1}\right)\right)$ is equivalent to that of $\left(\Xi\left(w, Y_{1}\right),\left(w^{\prime}, e\right)\right)$. Put $\Gamma=\Gamma\left(Y_{1}\right)=\left\{g \in G \mid Y_{1} \in \mathfrak{b}^{g}\right\}$. By $[\mathbf{2 8}, 3.1,(\mathrm{~b})$ and 3.3 , (c) $], \Xi\left(w, Y_{1}\right)$ is a union of irreducible components of $\Gamma \times \Gamma$. Hence, in order to show the irreducibility of $\left(T(B w B)^{\perp}, w^{\prime} Y_{1}\right)$, it suffices to show

$(5.16 .3)_{z} \quad(\Gamma(z), z)$ is irreducible

for $z=w^{\prime}$ and $e$. Let us consider the condition $(5.16 .3)_{z}$. Put $\mathfrak{n}=[\mathfrak{b}, \mathfrak{b}]$, define $q^{\prime}: G \rightarrow C$ by $q^{\prime}(g)={ }^{g} Y_{1}$, and let $q: \Gamma \rightarrow C \cap n$ be the base change of $q^{\prime}$. Since $q^{\prime}: G \rightarrow C$ is a surjective open morphism and it gives an (analytic) $Z_{G}\left(Y_{1}\right)$-principal bundle, $q: \Gamma \rightarrow C \cap n$ is also the same. From this fact, we can show that the image of each irreducible component of $\Gamma$ is an irreducible component of $C \cap \mathrm{r}$, and that $(5.16 .3)_{z}$ is equivalent to say that

$(5.16 .4)_{z}\left(q(\Gamma(z)),{ }^{z} Y_{1}\right)$ is irreducible.

\subsection{Local irreducibility of conormal bundles}

(1) Let $C$ be a nilpotent class in $\mathfrak{g}$ and $Y \in C \cap \mathfrak{n}$. If $2 \operatorname{dim}(\operatorname{ad}(\mathfrak{g}) Y \cap \mathfrak{n})=$ $\operatorname{dim} C$, then $(C \cap \mathrm{n}, Y)$ is irreducible.

(2) Assume (5.16.1), that

(5.17.1) ${ }_{z}{ }^{z} \Omega(z):=\left\{Y \in{ }^{z} \Omega \mid(C \cap \pi, Y)\right.$ is irreducible $\} \neq \phi$

for $z=w^{\prime}$ and $e$, and one of the following conditions.

$$
Z_{G}\left(Y_{1}\right) \text { is connected. }
$$


(5.17.3) There exists a Lie subalgebra $\mathfrak{q}$ of $\mathfrak{g}$ such that $\operatorname{dim}\left(\operatorname{ad}(\mathfrak{q}) \cdot{ }^{z} Y_{1}\right)=\frac{1}{2} \operatorname{dim} C$ for $z=w^{\prime}$ and $e$, and $\operatorname{ad}(\mathfrak{q})^{n} \cdot{ }^{w^{\prime}} Y_{1} \cup \operatorname{ad}(\mathfrak{q})^{n} \cdot Y_{1} \subset \mathfrak{b}$ for any $n$. Then $\left(\Lambda(w), w^{\prime} Y_{1}\right)$ is irreducible.

Remark 5.17.4. If $G=P G L_{l+1}, Z_{G}\left(Y_{1}\right)$ is always connected. In general, the component group of $Z_{G}\left(Y_{1}\right)$ is known [27, IV, 2.26] (classical types), [1] (exceptional types).

Proof. (1) Understand the intersection $C \cap \mathrm{n}$ scheme theoretically. Then $\operatorname{dim} T_{Y}(C \cap \mathfrak{n})=\operatorname{dim}\left(T_{Y} C \cap T_{Y} \mathfrak{n}\right)=\operatorname{dim}(\operatorname{ad}(\mathfrak{g}) Y \cap \mathfrak{n})=\frac{1}{2} \operatorname{dim} C=\operatorname{dim}(C \cap \mathfrak{n}) . \quad$ (The last equality follows from $[\mathbf{2 8}, 4.6]$ and $[\mathbf{2 6},(1)]$.) Hence $(C \cap \mathrm{n}, Y)$ is non-singular, and especially irreducible as an analytic space.

(2) First recall that $\Omega$ is an irreducible, locally closed hypersurface of $V^{*}\left(w^{\prime}\right)=\mathfrak{n}^{w^{\prime}} \cap \mathfrak{n}$. Especially ${ }^{z} \Omega \subset \mathfrak{n}$ for $z=w^{\prime}$ and $e$. Assume (5.16.1) and (5.17.1) for $z=w^{\prime}$ and $e$. Then $\Omega\left(w^{\prime}\right)$ and $\Omega(e)$ are open subsets of $\Omega$. Hence we can take $Y_{1}$ in (5.16.1) so that $Y_{1} \in \Omega\left(w^{\prime}\right) \cap \Omega(e)$. Then $\left(C \cap n,{ }^{z} Y_{1}\right)$ are irreducible for $z=w^{\prime}$ and $e$. Hence if (5.16.2) is satisfied, (5.16.4) $z$ are automatically satisfied. Let $\Gamma(z)$ be an irreducible component of $\Gamma$ containing $z\left(z=w^{\prime}\right.$ or $\left.e\right)$. Then $q(\Gamma(z))$ is an irreducible component of $C \cap n$ containing $q(z)={ }^{z} Y_{1}$. Because of the local irreducibility of $C \cap \mathrm{n}, q(\Gamma(z))$ does not depend on the choice of $\Gamma(z)$. If (5.17.2) is satisfied, $q^{-1} q(\Gamma(z))$ is irreducible, and hence $q^{-1} q(\Gamma(z))=\Gamma(z)$. Thus $\Gamma(z)$ is unique.

Next assume (5.17.3). Let $Q$ be the connected subgroup of $G$ whose Lie algebra is $\mathfrak{q}$. Then by the latter half of (5.17.3), ${ }^{Q z} Y_{1} \subset \mathfrak{b}$ for $z=w^{\prime}$ and $e$, where ${ }^{Q z} Y_{1}=\left\{{ }^{q z} Y_{1} \mid q \in Q\right\}$. Since every irreducible component of $C \cap n$ is of the same dimension [26], $\operatorname{dim} q(\Gamma(z))=\operatorname{dim}(C \cap \mathrm{n})$. On the other hand, $\operatorname{dim}{ }^{Q z} Y_{1}=$ $\operatorname{dim}\left(\operatorname{ad}(\mathfrak{q}) \cdot{ }^{z} Y_{1}\right)=\frac{1}{2} \operatorname{dim} C=\operatorname{dim}(C \cap \mathfrak{n})$. Hence $\operatorname{dim}{ }^{Q_{z}} Y_{1}=\operatorname{dim} q(\Gamma(z)),{ }^{Q_{z}} Y_{1}$ is open dense in $q(\Gamma(z)), Q z Z_{G}\left(Y_{1}\right)$ is open dense in $q^{-1} q(\Gamma(z))$, and consequently, $\left(q^{-1} q(\Gamma(z)), z\right)$ is irreducible. Thus $\Gamma(z)$ is unique.

5.18. Holonomy diagram To each $w \in\left(W_{I^{\prime}} \backslash W / W_{I}\right)_{l}$ such that $\Lambda(w) \subset \mathbf{W}$, associate a vertex labeled $w$, and connect two vertexes associated to $w$ and $w^{\prime}$ if $\operatorname{dim} \Lambda(w) \cap \Lambda\left(w^{\prime}\right)=\operatorname{dim} \Lambda(w)-1$. Thus we obtain a graph, which we shall call the holonomy diagram of $(\mathfrak{g}, I)$. (Sometimes we call a subgraph of the holonomy diagram a holonomy diagram.) Sometimes we write $\operatorname{ord}_{\Lambda(w)} f^{s}$ beside the vertex associated to $w$, and the intersection exponent $(\mu: v)$ beside the edge corresponding to the intersection. Put

$$
W_{0}(I)=\left\{w \in\left(W_{I^{\prime}} \backslash W / W_{I}\right)_{l} \mid \Lambda(w) \subset \mathbf{W}_{0}\left(f^{\delta}\right)\right\} .
$$

Then the vertexes of the holonomy diagram are parametrized by $W_{0}(I)$. 
5.19. Local $\boldsymbol{b}$-functions Let $S-I=\left\{i_{1}, \cdots, i_{k}\right\}, \quad I=\left\{i_{k+1}, \cdots, i_{l}\right\}, \quad \underline{s}=$ $\left(s_{i_{1}}, \cdots, s_{i_{k}}\right), w \in W_{0}(I)$ and $i \in S-I$. Assume that there exist a micro-differential operator $Q_{i}(\underline{s}) \in \mathscr{E}[\underline{s}]=\mathscr{E} \otimes_{\mathbf{C}} \mathbf{C}[\underline{s}]$ whose principal symbol is independent of $\underline{s}$ and invertible in a neighbourhood of a generic point of $\Lambda(w)$, and a polynomial $b_{w, i}(\underline{s}) \in \mathbf{C}[\underline{s}]=\mathbf{C}\left[s_{i_{1}}, \cdots, s_{i_{k}}\right]$ such that $f_{i} f^{\lambda+0 \delta}=b_{w, i}(\lambda) Q_{i}(\lambda) f^{\lambda+0 \delta}$ for any $\lambda=\sum_{i \in S-I} \lambda_{i} \varpi_{i}$. Such a polynomial $b_{w, i}$ is called the local $b$-function. If $\Lambda(w)$ is a good Lagrangian (cf. (5.4)), then such $Q_{i}(\underline{s})$ and $b_{w, i}(i \in S-I$ ) exist and $b_{w, i}(i \in S-I)$ are unique up to non-zero constant multiples [24], [10].

5.20. Calculation of local $\boldsymbol{b}$-functions The local $b$-functions can be calculated using the holonomy diagram as follows. Let $\Lambda(w)$ and $\Lambda\left(w^{\prime}\right)$ be good Lagrangian varieties which intersect in codimension one. Let $(\mu: v)$ be their intersection exponents,

$$
\operatorname{ord}_{\Lambda(w)} f^{\lambda+0 \delta}=\sum_{i \in S-I} m_{i} \lambda_{i}-\frac{\mu_{w}}{2}, \quad \text { and } \quad \operatorname{ord}_{\Lambda\left(w^{\prime}\right)} f^{\lambda+0 \delta}=-\sum_{i \in S-I} m_{i}^{\prime} \lambda_{i}-\frac{\mu_{w^{\prime}}}{2} .
$$

Assume that $m_{i} \leq m_{i}^{\prime}$, that some irreducible component of $\Lambda(w) \cap \Lambda\left(w^{\prime}\right)$, say $\Delta$, is not contained in any $\Lambda\left(w^{\prime \prime}\right)\left(w^{\prime \prime} \in W_{0}(I) \backslash\left\{w, w^{\prime}\right\}\right)$, and that $\Lambda(w)$ and $\Lambda\left(w^{\prime}\right)$ are locally irreducible as analytic spaces at a generic point of $\Delta$. Then up to a non-zero constant multiple, we have

$$
\frac{b_{w^{\prime}, i}(\lambda)}{b_{w, i}(\lambda)}=\prod_{j=0}^{v}\left[\frac{1}{v+1}\left(\operatorname{ord}_{\Lambda(w)} f^{\lambda+0 \delta}-\operatorname{ord}_{\Lambda\left(w^{\prime}\right)} f^{\lambda+0 \delta}\right)+\frac{\mu+2 j}{2(v+\mu)}\right]^{\frac{m_{l}^{\prime}-m_{2}}{v+1}},
$$

where $[x]^{j}=x(x+1) \cdots(x+j-1)$ for $j>0$ and $[x]^{0}=1$. Especially, if $(\mu, v)=(1,0)$, then

$$
\frac{b_{w^{\prime}, i}(\lambda)}{b_{w, i}(\lambda)}=\left[\operatorname{ord}_{\Lambda(w)} f^{\lambda+0 \delta}-\operatorname{ord}_{\Lambda\left(w^{\prime}\right)} f^{\lambda+0 \delta}+\frac{1}{2}\right]^{m_{i}^{\prime}-m_{\imath}} .
$$

(Since $\mathbf{W}_{0}$ has exactly two irreducible components in the analytic sense in a neighbourhood of a generic point of $\Delta$, we can apply [24, Theorem 7.1] to our situation. By $[\mathbf{1 0}, 4.5]$, the remaining argument of $[24, \S 7]$, with an obvious modification, also works in our situation.)

5.21. After submitting the first draft, the author learnt from M. Kashiwara a way to show that an irreducible (germ of) analytic space $(\Delta, q)(\subset \mathbf{W})$ of dimension $\operatorname{dim} G-1$ is contained in at most two irreducible components, say $\Lambda$ and $\Lambda^{\prime}$, of $\left(\mathbf{W}_{0}, q\right)$. This algorithm works only if $\Lambda$ and $\Lambda^{\prime}$ regularly intersect each other, i.e., with the intersection exponent $(\mu: v)=(1: 0)$. Thus, for example in (7.1) below, it does not work for the intersection of $1(121)$ and $\Lambda(12121)$, but it does work and simplifies the argument for the other intersections. 
Here we include this algorithm by permission of $\mathrm{M}$. Kashiwara, to whom the author is very grateful.

Put $H:=P^{\prime} \times P$ and $H_{0}:=\left\{\left(p^{\prime}, p\right) \in P^{\prime} \times P \mid\left(w_{S} \delta\right)\left(p^{\prime}\right) \delta(p)^{-1}=1\right\}$. Let $\Delta$ be an $H$-stable closed subvariety of $\mathbf{W}$ of dimension $n-1(n:=\operatorname{dim} G)$ which contains a dense $H_{0}$-orbit $H_{0} \cdot q$;

$$
\overline{H_{0} \cdot q}=\Delta
$$

Let $T_{0}$ be a maximal torus of the isotropy group $H_{q}$. As in the proof of $[\mathbf{2 4}, 6.6]$, we can show that $\mathbf{W}$ is non-singular at $q$. Hence by $[\mathbf{1 0}, 3.1]$, we can find a local coordinate system $\left\{x_{1}, \cdots, x_{n+1}\right\}$ of $(\mathbf{W}, q)$ such that $x_{j}(q)=0$ $(1 \leq j \leq n+1), x_{1} \equiv x_{2} \equiv 0$ on $\Delta$, and each $x_{j}$ is relatively $T_{0}$-invariant with a character $\phi_{j}$. Put $F:=\left\{x_{3}=\cdots=x_{n+1}=0\right\}$. Then

$$
\begin{aligned}
& \left(x_{1}, x_{2}\right): F \rightarrow \mathbf{C}^{2} \text { maps } q \text { to }(0,0) \text {, étale in a neighbourhood of } q \text {, and } \\
& T_{0} \text {-equivariant if we consider the diagonal } T_{0} \text {-action diag }\left(\phi_{1}, \phi_{2}\right) \text { on } \mathbf{C}^{2} \text {. }
\end{aligned}
$$

Assume that

$$
\phi_{1}^{i}=\phi_{2}^{j} \quad \text { and } \quad i, j \in \mathbf{Z}_{\geq 0} \Longleftrightarrow(i, j)=(0,0) .
$$

5.21.4. Let us show that there is no $T_{0}$-stable analytic curve in $\mathbf{C}^{2}$ containing $(0,0)$, except for the coordinate axes.

Assume the contrary, and let $C$ be a $T_{0}$-stable curve in $\mathbf{C}^{2}$ containing $(0,0)$, different from the coordinate axes. We may assume that $(C,(0,0))$ is irreducible. Let $\varphi\left(x_{1}, x_{2}\right)=\sum_{i, j \geq 0} a_{i j} x_{1}^{i} x_{2}^{j}$ be a defining equation of $(C,(0,0))$. Since any transform of $\varphi$ by $\left(T_{0}, e\right)$ is also a defining equation of $C, \varphi$ is relatively $T_{0}$-invariant. Hence $\phi_{1}^{i} \phi_{2}^{j}$ does not depend on the terms $x_{1}^{i} x_{2}^{j}$ appearing in $\varphi$. By (5.21.3), it follows from this remark that (1) any $x_{2}^{j}(j \geq 0)$ do not appear in $\varphi$ or (2) any $x_{1}^{i}(i \geq 0)$ do not appear in $\varphi$. In other words, $\varphi$ is divisible by $x_{1}$ or $x_{2}$. But $\varphi^{-1}(0)=C$ is an irreducible curve other than the coordinate axes. Thus we get a contradiction.

5.21.5. Let us show that there are at most two irreducible components of $\left(\mathbf{W}_{0}, q\right)$ containing 4 . Let $(\Lambda, q)$ be such an irreducible component. Let $C_{i}$ $(i=1,2)$ be the (local) analytic curve contained in $(F, q)$ and defined by $x_{i}=0$ $(i=1,2)$. Thus $\Lambda \cap F=C_{1}$ or $C_{2}$ by (5.21.2) and (5.21.4). If ' $=C_{i}$ ', then $\Lambda$ contains $(H, e) \cdot C_{i}$. The latter contains $C_{i}$ and $(\Delta, q)=(H, e) \cdot q$, and hence is of dimension at least $n$. Thus $\Lambda=(H, e) \cdot C_{i}$ for $i=1,2$.

5.21.6. To see if (5.21.3) is satisfied, it suffices to calculate $\left\{\phi_{1}, \phi_{2}\right\}$. In the Grothendieck group of $T_{0}$-modules

$$
\begin{aligned}
{\left[\phi_{1}\right]+\left[\phi_{2}\right] } & =\left[T_{q} F\right]=\left[T_{q} \mathbf{W}\right]-\left[T_{q} \Delta\right] \\
& =\left[\left(T_{q} \Delta\right)^{\perp}\right]-\left[T_{q} \Delta\right] \text { by }(5.21 .1)
\end{aligned}
$$




$$
=\left[T_{q}\left(T^{*} G\right)\right]-\left[T_{q} \Delta\right]^{*}-\left[T_{q} \Delta\right] .
$$

As for the third equality, see the proof of $[24,6.6]$. The orthogonal complement ()$^{\perp}$ is considered with respect to the canonical bilinear form on $T_{q}\left(T^{*} G\right)$. The contragradient representation and also the corresponding element of the Grothendieck group is indicated by the superscript *. Furhter assume that $\Delta$ is contained in a Lagrangian variety $\Lambda$ which is non-singular at $q$. Then

$$
\left[T_{q} \Lambda\right]=\left[\left(T_{q} \Lambda\right)^{\perp}\right]=\left[T_{q}\left(T^{*} G\right)\right]-\left[T_{q} \Lambda\right]^{*},
$$

and hence

$$
\left[\phi_{1}\right]+\left[\phi_{2}\right]=\left(\left[T_{q} \Lambda\right]-\left[T_{q} \Lambda\right]\right)^{*}+\left(\left[T_{q} \Lambda\right]-\left[T_{q} \Lambda\right]\right) .
$$

Then $\phi_{1}=\phi_{2}^{-1}$, and (5.21.3) is equivalent to the following condition.

The $T_{0}$-action on $T_{q} \Lambda / T_{q} \Delta$ is non-trivial.

Restating (5.21.1) and (5.21.7) using colocalization, we get the following.

5.22. Intersection of conormal bundles (6) Assumptions and notations be as in (5.7). Let $T_{0}$ be a maximal torus of the isotropy group $G(w)_{Y_{2}}$. Assume that $T_{0}$ acts on $V^{*}(w) /(\operatorname{adg}(w)) Y_{2}$ non-trivially. Then $w Y_{2}$ is not contained in any irreducible component of $\mathbf{W}_{0}$ other than $\Lambda(w)$ or $\Lambda\left(w^{\prime}\right),\left(\Lambda(w), w Y_{2}\right)$ and $\left(\Lambda\left(w^{\prime}\right), w Y_{2}\right)$ are irreducible and non-singular, and their intersection exponent is $(\mu: v)=(1: 0)$.

\section{§6. Kazhdan-Lusztig Conjectures}

6.0. In [18] and [19], Kazhdan and Lusztig made several conjectures, one of which has been settled [2], [4]. Using or assuming these assertions, we can get useful information on the micro-local structure of $\mathscr{D} f^{\lambda+0 \delta}$. In order to state their conjectures, we need to review [18].

6.1. Kazhdan-Lusztig polynomials The Kazhdan-Lusztig polynomials $P_{y, w}(q)(y, w \in W)$ are polynomials in $q$ with non-negative integral coefficients, and $P_{y, w}(0)=1$. They can be calculated in the following way [18]. (1) If $y \nless w$, then $P_{y, w}=0$. (2) If $y \leq w$ and $l(w)-l(y) \leq 2$, then $P_{y, w}=1$. (3) If $y \lessgtr w$, then $P_{y, w}(q)$ can be expressed as $P_{y, w}(q)=\sum_{i=0}^{l(w)-l(y)-1} \mu_{y, w}(i) q^{\frac{i}{2}}$ with $\mu_{y, w}(i) \in \mathbf{Z}$, where $\mu_{y, w}(i)=0$ for odd $i$. Let $\mu(y, w)=\mu_{y, w}(l(w)-l(y)-1)$. If $y \leq s y, w \leq s w$ and $s \in S$, then

$$
P_{y, s w}=q P_{s y, w}+P_{y, w}-\sum_{\substack{z \\ z \leq s z \\ y \leq s z<w}} \mu(s z, w) q^{\frac{1}{2}(l(w)-l(z))} P_{y, s z}
$$

Thus we can calculate $P_{y, w}$ inductively. Note that $\mu(y, w)=0$ if $l(w)-l(y)$ is even. 
6.2. Edges of holonomy diagrams In order to calculate holonomy diagrams, we need to find codimension one intersections of conormal bundles $\Lambda(w)$. We shall find such intersections by using the technique developped in $\S 5$. Sometimes, the following conjecture of Kazhdan-Lusztig [18, p. 167, $\ell$. $1-$ p. $168, \downarrow \ell .1]$ is useful to find candidates.

Conjecture 6.2.1. Assume that $G$ is of type $A_{l}, y, w \in W$ and $y<w$. Then $\operatorname{dim} \Lambda(y) \cap \Lambda(w)=\operatorname{dim} \Lambda(w)-1$ if and only if $\mu(y, w) \neq 0$.

As is noted in [18], this conjecture does not hold if $G$ is not of type $A_{l}$. For example, the edges $12121-121$ and $121-1$ in the holonomy diagram of $\left(G_{2}, 2\right)$ calculated in (7.1) below can not be predicted in this way. (For type $G_{2}, \mu(y, w)=1$ if $l(w)-l(y)=1$, and $\mu(y, w)=0$ otherwise.)

6.3. The following assertion was conjectured by Kazhdan-Lusztig [18, 1.5], and proved by Brylinski-Kashiwara [4] and Beilinson-Bernstein [2].

Lemma 6.3.1. Let $M_{w}$ be the Verma module with the highest weight $-w \rho-\rho$, and $L_{w}$ its simple quotient. Then $\left[L_{w}\right]=\sum_{y}(-1)^{l(y)+l(w)} P_{y, w}(1)\left[M_{y}\right]$, and $\left[M_{w}\right]=\sum_{y} P_{w_{s} w, w_{s} y}(1)\left[L_{y}\right]$, where $\left[L_{w}\right]$ etc. are the element of the Grothendieck group corresponding to $L_{w}$ etc.

6.4. Let $X(w)=B w B / B, X=G / B$ and $\mathscr{L}_{w}=\mathscr{D}_{X} \otimes_{U(\mathrm{~g})} L_{w}$. The following assertion was originally conjectured by Kazhdan-Lusztig $[19, \S 7]$ and expressed in the following form by Kashiwara-Tanisaki [17].

Conjecture 6.4.1. If $G$ is of type $A_{l}$, then $\mathbf{C h}\left(\mathscr{L}_{w}\right)=\left[T X(w)^{\perp}\right]$, where $\left[T X(w)^{\perp}\right]$ denotes the algebraic cycle determined by $T X(w)^{\perp}$.

Remark 6.4.2. If $G$ is not of type $A_{l}$, an analogous assertion does not hold [17]. Cf. (7.3) below.

6.5. Vertexes of holonomy diagrams In (5.5.2) and (5.7), we have given methods to show that $\Lambda(w) \subset \mathbf{W}_{0}\left(f^{\delta}\right)$ or not. Here we give another method to predict that $\Lambda(w) \subset \mathbf{W}_{0}\left(f^{\delta}\right)$, based on (6.3.1) and (6.4.1).

Lemma 6.5.1. (1) Let $y \in\left(W_{I^{\prime}} \backslash W / W_{I}\right)_{l}$. If

$$
\sum_{x \in W_{I}}(-1)^{l(x)} P_{x, w_{s}, y}(1) \neq 0,
$$

then $\Lambda(y) \subset \mathbf{W}_{0}\left(f^{\delta}\right)$, i.e., $y \in W_{0}(I)$. (2) Let $G$ be of type $A_{l}$ and assume the validity of the conjecture (6.4.1). Then (6.5.2) is a necessary and sufficient condition in order that $y \in W_{0}(I)$.

Proof. By $[8,7.17],[V(w, 0, p(I))]=\sum_{x \in W_{I}}(-1)^{l(x)}\left[M_{u^{\prime} x}\right]$ for $w \in\left(W / W_{I}\right)_{l}$. By (6.3.1), $[V(w, 0, \mathfrak{p}(I))]=\sum_{y} c(y, w)\left[L_{y}\right]$, where $c(y, w)=\sum_{x \in W_{I}}(-1)^{l(x)} \times$ 
$P_{w_{s} w x, w_{s} y}(1) . \quad$ Put cd $(w)=l\left(w_{S}\right)-l(w) . \quad$ By $\left[8,3.4\right.$. and 6.6], Ch $H_{B w P / B}^{c d(w)}\left(\mathcal{O}_{G / B}\right)$ $=\sum_{y} c(y, w) \mathbf{C h}\left(\mathscr{L}_{y}\right)$. Since $\mathscr{L}_{y}$ is the simple quotient of $H_{B y B / B}^{c d(y)}\left(\mathscr{O}_{G / B}\right), \mathbf{C h}\left(\mathscr{L}_{y}\right)$ contains the conormal bundle of $B y B / B$ with multiplicity one. By $[8,(9.11 .1)]$, $p_{X}^{*} H_{B w P / B}^{c d(w)}\left(\mathcal{O}_{G / B}\right)=H_{B w P}^{c d(w)}\left(L_{(w w s)^{-1}}^{*} \mathscr{D}_{G} f^{0 \delta}\right)$ in the notation of $[8] . \quad\left(L_{g}(x)=g x\right.$, and $p_{X}: G \rightarrow G / B=X$ is the projection. See (5.4) for $\mathscr{D}_{G} f^{0 \delta}$.) Hence, taking $w=w_{S}$, we get

$$
\operatorname{Ch} H_{B w_{s} P}^{0}\left(\mathscr{D}_{G} f^{0 \delta}\right)=\sum_{y} c\left(y, w_{S}\right) \mathbf{C h}\left(p_{X}^{*} \mathscr{L}_{y}\right) .
$$

By (2.6), $B w_{S} P=P^{\prime} w_{S} P=G \backslash \bigcup_{i \in S \backslash I} f_{i}^{-1}(0)$. Hence $H_{B w_{s} P}^{0}\left(\mathscr{D}_{G} f^{0 \delta}\right)=\left(\mathscr{D}_{G} f^{0 \delta}\right)$ $\left[\left(f^{\delta}\right)^{-1}\right]$. But by (1.4) and (1.5), $\mathbf{C h}\left(\mathscr{D}_{G} f^{0 \delta}\right)\left[\left(f^{\delta}\right)^{-1}\right]=\mathbf{C h} \mathscr{D} f^{0 \delta}$. Hence (6.5.3) implies that

$$
\mathbf{C h} \mathscr{D}_{G} f^{0 \delta}=\sum_{y} c\left(y, w_{S}\right) \mathbf{C h}\left(p_{X}^{*} \mathscr{L}_{y}\right)
$$

Hence, if $c\left(y, w_{S}\right) \neq 0$, then $\Lambda(y) \subset \mathrm{Ch}\left(p_{X}^{*} \mathscr{L}_{y}\right) \subset \mathrm{Ch}\left(\mathscr{D}_{G} f^{0 \delta}\right)=\mathbf{W}_{0}\left(f^{\delta}\right)$. If $G$ is of type $A_{l}$ and if we assume the validity of the conjecture (6.4.1), then (6.5.4) becomes $\mathbf{C h} \mathscr{D}_{G} f^{0 \delta}=\sum_{y} c(y, w)[\Lambda(y)]$. Hence $c(y, w) \neq 0 \Leftrightarrow \Lambda(y) \subset \mathbf{W}_{0}\left(f^{\delta}\right)$.

\section{§7. Examples (2)}

7.0. Here we calculate $b$-functions of semi-invariants of some $(\mathfrak{g}, I)$ by applying the method developped in $\S 5$ and $\S 6$.

\section{1. $\left(G_{2}, 2\right) \quad \underset{\alpha_{1}}{\bullet} \underset{\alpha_{2}}{Ð}$} and

(Cf. (4.0).) Let $g$ be the Lie algebra of type $G_{2}$ and $I=\left\{r_{1}\right\}$. Then $I^{\prime}=I$

$$
\left(W_{I^{\prime}} \backslash W / W_{I}\right)_{l}=\{1,121,12121,121212\} .
$$

We give the sets $R(w)=w^{-1} R_{+} \cap R_{+}$and $R^{\prime}(w)=w^{-1} R_{I^{\prime},-} \cup R_{I_{1}-}$ in the following table.

\begin{tabular}{|cccc|}
\hline 1 & 121 & 12121 & 121212 \\
\hline$\alpha_{2}$ & $\alpha_{2}$ & $\alpha_{2}$ & \\
$\alpha_{1}+\alpha_{2}$ & $\alpha_{1}+\alpha_{2}$ & & \\
$2 \alpha_{1}+\alpha_{2}$ & & & \\
$3 \alpha_{1}+\alpha_{2}$ & & & \\
$3 \alpha_{1}+2 \alpha_{2}$ & $3 \alpha_{1}+2 \alpha_{2}$ & & \\
\hline$-\alpha_{1}$ & $-\alpha_{1}$ & $-\alpha_{1}$ & $-\alpha_{1}$ \\
$\alpha_{1}$ & $2 \alpha_{1}+\alpha_{2}$ & $\alpha_{1}+\alpha_{2}$ & $\alpha_{1}$ \\
\hline
\end{tabular}


The top line gives $w$. The boxed part gives $R(w)$. The second line from the bottom gives $R_{I_{-}-}$. The last line gives $w^{-1} R_{I^{\prime},-}$. Thus the last two lines consist $R^{\prime}(w)$. Since $V^{*}(w)=\sum_{\alpha \in R(w)} \mathfrak{g}(\alpha)$ and Lie $(G(w))=\mathrm{t}+\sum_{\alpha \in R(w) \cup R^{\prime}(w)} \mathfrak{g}(\alpha)$, we can calculate the orbit structure of the colocalizations $\left(G(w), V^{*}(w)\right)$, a part of which we give in the following table.

$$
\begin{array}{cccc}
1 & X_{\alpha_{1}+\alpha_{2}}+X_{2 \alpha_{1}+\alpha_{2}} & X_{\alpha_{1}+\alpha_{2}} & 3 \alpha_{1}+\alpha_{2} \\
121 & X_{\alpha_{1}+\alpha_{2}} & X_{\alpha_{2}}+X_{3 \alpha_{1}+2 \alpha_{2}} & \alpha_{1}+\alpha_{2} . \\
12121 & X_{\alpha_{2}} & 0 & \alpha_{2}
\end{array}
$$

The first column gives $w$. The second column gives points which belong to the open orbits of $\left(G(w), V^{*}(w)\right)$. The third column gives points which belong to codimension one orbits of $\left(G(w), V^{*}(w)\right)$. In the present case, it happens that each row contains only one element in the third column, but it is not a general feature. If $Y_{1}$ belongs to a codimension one orbit of $\left(G(w), V^{*}(w)\right)$, then $V^{*}(w) /\left(\mathfrak{g}(w) \cdot Y_{1}\right)$ is a one dimensional vector space on which $\{H \in \mathfrak{t} \mid$ ad $\left.(H) Y_{1} \in \mathbf{C} Y_{1}\right\}=: \mathrm{t}_{1}$ operates. The last column gives the corresponding characters of $t_{1} \cdot\left(\alpha_{2}\right.$ etc. means $\alpha_{2} \mid t_{1}$ etc. $)$

Since each element, say $Y_{0}$, contained in the second column are of the form $X_{\gamma_{1}}+X_{\gamma_{2}}+\cdots$ with linearly independent $\gamma_{1}, \gamma_{2}, \cdots$, we can find an element $A_{0}$ in $\mathrm{t}$ such that $\left\langle\gamma_{1}, A_{0}\right\rangle=\left\langle\gamma_{2}, A_{0}\right\rangle=\cdots=1$, i.e., $\left(\operatorname{ad} A_{0}\right) Y_{0}=Y_{0}$. Since $w_{S}=-1$ in the present case, (5.5) gives

$$
\operatorname{ord}_{\Lambda(w)} f_{2}^{\lambda_{2}}=-\left\langle w^{-1} \varpi_{2}+\varpi_{2}, A_{0}\right\rangle \lambda_{2}-\sum_{\alpha \in R(w)}\left\langle\chi, A_{0}\right\rangle+\frac{1}{2} \operatorname{card} R(w) .
$$

Here and below, we write $f^{\lambda}$ for $f^{\lambda+0 \delta}$ if there is no fear of confusion. Since $\varpi_{2}=3 \alpha_{1}+2 \alpha_{2}, w^{-1} \varpi_{2}+\varpi_{2}$ is equal to

$$
\begin{array}{ll}
6 \alpha_{1}+4 \alpha_{2}=2 \cdot\left(\alpha_{1}+\alpha_{2}\right)+2 \cdot\left(2 \alpha_{1}+\alpha_{2}\right) & \text { if } w=1 \\
3 \alpha_{1}+3 \alpha_{2}=3 \cdot\left(\alpha_{1}+\alpha_{2}\right) & \text { if } w=121 \\
\alpha_{2}=1 \cdot \alpha_{2} & \text { if } w=12121
\end{array}
$$

Thus in each case, $w^{-1} \varpi_{2}+\varpi_{2}$ can be expressed as a linear combination of $\gamma$ 's such that $X_{\gamma}$ appears in the expression of $Y_{0}$. Hence $\left\langle w^{-1} \varpi_{2}+\varpi_{2}, A_{0}\right\rangle$ is equal to the sum of the coefficients appeared in the right hand sides of (7.1.2), and independent of a special choice of $A_{0}$. The sum $\sum_{\alpha \in R\left(w^{\prime}\right)} \chi$ is also expressed as a linear combination of $\gamma^{\prime}$ 's as above, and $\sum_{\alpha \in R(w)}\left\langle\alpha, A_{0}\right\rangle$ can be determined in the same way. The order of $f_{2}^{s}$ at $\Lambda(w)$ is $-4 s-\frac{7}{2}$ if $w=1$, $-3 s-\frac{5}{2}$ if $w=121,-s-\frac{1}{2}$ if $w=12121$, and 0 if $w=121212$.

Next, taking $Y_{1}$ from the third column of (7.1.1), and assuming that 
$w Y_{1} \in w V^{*}(w) \subset \Lambda(w)$ lies in another conormal bundle $\Lambda\left(w^{\prime}\right)$ and that the assumptions in (5.6) are satisfied, we shall calculate the intersection exponents $(\mu: v)$ of $\Lambda(w)$ and $\Lambda\left(w^{\prime}\right)$. Take $A_{1} \in \mathrm{t}$ so that $\left(\operatorname{ad} A_{1}\right) Y_{1}=Y_{1}$. The intersection exponents can be calculated by $\left\langle\eta, A_{1}\right\rangle=\mu /(\mu+v)$, where $\eta$ is the character contained in the last column of (7.1.1). For $w=121, \eta$ can be expressed as a linear combination of $\gamma \in R$ such that $X_{\gamma}$ appears in the expression of $Y_{1}$. Hence the value of $\left\langle\eta, A_{1}\right\rangle$ does not depend on the special choice of $A_{1}$ and is equal to $\frac{2}{3}$ if $w=121$. But for $w=1$ or 12121 , the value of $\left\langle\eta, A_{1}\right\rangle$ does depend on the choice of $A_{1}$. Hence $(\mu, v)$ is equal to $(1,0)$ if $w=1,(2,1)$ if $w=121$, and $(1,0)$ if $w=12121$.

We can show that the assumptions of (5.7) are satisfied if we take as $Y_{2}$ the element of the third column of (7.1.1) for $w=1$ or $w=12121$.

Let us determine which $\Lambda(w)$ and $\Lambda\left(w^{\prime}\right)$ have a codimension one intersection, i.e., which pair $\left(w, w^{\prime}\right)$ is linked by an edge in the holonomy diagram. Obviously, we have an edge $12121-121212$. Let us find elements in $\Lambda(121) \cap \Lambda(1)$ by the algorithm of (5.10). Put $w_{0}=121, w_{1}=12$, and $w_{2}=1$. Then $\gamma_{0}=\alpha_{1}$, $\gamma_{1}=\alpha_{2}, E_{0}=\left\{\alpha_{2}, \alpha_{1}+\alpha_{2}, 3 \alpha_{1}+2 \alpha_{2}\right\}, E_{1}=\left\{2 \alpha_{1}+\alpha_{2}, 3 \alpha_{1}+\alpha_{2}, 3 \alpha_{1}+2 \alpha_{2}\right\}$, and $E_{2}=\left\{2 \alpha_{1}+\alpha_{2}, 3 \alpha_{1}+\alpha_{2}, 3 \alpha_{1}+2 \alpha_{2}\right\}$. Hence $X_{2 \alpha_{1}+\alpha_{2}}+X_{3 \alpha_{1}+\alpha_{2}} \in r_{1}^{-1}$. $(\Lambda(121) \cap \Lambda(1)) \subset V\left(r_{1}\right)$. Cf. (5.10.1). As is easily seen, this element belongs to a codimension one orbit of $\left(G\left(r_{1}\right), V\left(r_{1}\right)\right)$. Since $\alpha_{1}^{\vee} \in\left[\mathfrak{g}\left(\alpha_{1}\right), \mathfrak{g}\left(\alpha_{1}\right)\right]$, we get rank $G\left(r_{1}\right) /\left[G\left(r_{1}\right), G\left(r_{1}\right)\right] \leq 1$ and consequently, $\left(G\left(r_{1}\right), V\left(r_{1}\right)\right)$ has at most one orbit of codimension one. Hence $X_{\alpha_{1}+\alpha_{2}}$ (the representative of the codimension one orbit given in (7.1.1)) and $X_{2 \alpha_{1}+\alpha_{2}}+X_{3 \alpha_{1}+\alpha_{2}}$ belong to the same orbit. Since $r_{1}^{-1} \cdot(\Lambda(121) \cap \Lambda(1))\left(\subset V\left(r_{1}\right)\right)$ is $G\left(r_{1}\right)$-stable, $\Lambda(1)$ and $\Lambda(121)$ have codimension one intersection with the intersection exponents $(\mu: v)=(1: 0)$, as we have already calculated. (Actually $\left(G\left(r_{1}\right), V\left(r_{1}\right)\right)$ has five orbits represented by $X_{\alpha_{1}+\alpha_{2}}+X_{2 \alpha_{1}+\alpha_{2}}(\operatorname{dim}=5), X_{\alpha_{1}+\alpha_{2}}(\operatorname{dim}=4), X_{\alpha_{2}} \quad(\operatorname{dim}=3), \quad X_{3 \alpha_{1}+2 \alpha_{2}}$ $(\operatorname{dim}=1)$, and 0 .)

Let us find elements in $\Lambda(12121) \cap \Lambda(121)$ by using algorithm of (5.10) again. Put $w_{0}=12121, w_{1}=1212$, and $w_{2}=121$. Then $\gamma_{1}=\alpha_{1}, \gamma_{2}=\alpha_{2}$, $E_{0}=\left\{\alpha_{2}\right\}, E_{1}=\left\{3 \alpha_{1}+\alpha_{2}\right\}$ and $E_{2}=\left\{3 \alpha_{1}+2 \alpha_{2}\right\}$. Next, put $w_{0}=12121$, $w_{1}=2121$ and $w_{2}=121$. Then $\gamma_{0}=1212\left(\alpha_{1}\right)=\alpha_{1}+\alpha_{2}, \gamma_{1}=121\left(\alpha_{2}\right)=3 \alpha_{1}+$ $2 \alpha_{2}, E_{0}=\left\{\alpha_{2}\right\}, E_{1}=\left\{\alpha_{2}\right\}$, and $E_{2}=\left\{\alpha_{2}\right\}$. Hence

$$
\mathfrak{g}\left(3 \alpha_{1}+2 \alpha_{2}\right) \cup \mathfrak{g}\left(\alpha_{2}\right) \subset w^{\prime-1}\left(\Lambda(w) \cap \Lambda\left(w^{\prime}\right)\right),
$$

where $w=12121$ and $w^{\prime}=121$. By (5.12), $\operatorname{Grass}_{1}\left(w^{\prime-1}\left(\Lambda(w) \cap \Lambda\left(w^{\prime}\right)\right)\right.$ contains a $G\left(w^{\prime}\right)$-stable connected set, say $Z$, containing $\left\{\mathfrak{g}\left(3 \alpha_{1}+2 \alpha_{2}\right), \mathfrak{g}\left(\alpha_{2}\right)\right\}$. Since the orbits of $\left(G\left(w^{\prime}\right), V^{*}\left(w^{\prime}\right)\right)$ are represented by $X_{\alpha_{1}+\alpha_{2}}(\operatorname{dim}=3), X_{3 \alpha_{1}+2 \alpha_{2}}+X_{\alpha_{2}}$ $(\operatorname{dim}=2), X_{3 \alpha_{1}+2 \alpha_{2}}(\operatorname{dim}=1), X_{\alpha_{2}}(\operatorname{dim}=1)$ and 0 , the $G\left(w^{\prime}\right)$-stable subset $\cup Z$ of $V^{*}\left(w^{\prime}\right)$ should contain the orbit of codimension one. Hence $\Lambda(w)$ and $\Lambda\left(w^{\prime}\right)$ intersect in codimension one, and its intersection exponent is $(2: 1)$, as we have 


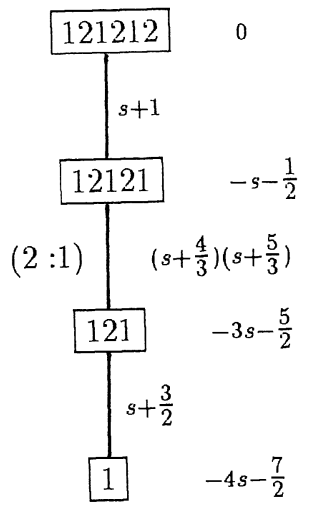

(Figure 3)

already calculated assuming the necessary local irreducibility, whose proof we shall give afterwards. Thus we get a holonomy diagram.

Here we have written the ratio of local $b$-functions besides the respective edges. Let us show that this is the holonomy diagram. By (5.7), we can show that $W_{0}(I)=\{121212,12121,121,1\}$. Let $\Delta$ be the closure of the $B \times B$-orbit of $121 \cdot\left(X_{3 \alpha_{1}+2 \alpha_{2}}+X_{\alpha_{2}}\right)$ in $T^{*} G$. We have shown that $\Delta \subset A(12121) \cap \Lambda(121)$ and $\operatorname{dim} \Delta=\operatorname{dim} G-1$. By (5.15), $\Delta \not \subset \Lambda(1)$. Obviously $\Delta \not \subset \Lambda(121212)$. Next, let $\Delta$ be the closure of the $B \times B$-orbit of $r_{1} \cdot\left(X_{2 \alpha_{1}+\alpha_{2}}+X_{3 \alpha_{1}+\alpha_{2}}\right)$ in $T^{*} G$. We have shown that $\Delta \subset \Lambda(121) \cap \Lambda(1)$ and $\operatorname{dim} \Delta=\operatorname{dim} G-1$. Obviously $\Delta \not \subset \Lambda(121212)$. Let us show that $\Delta \not \subset \Lambda(12121)$, using (5.14). Since $\overline{\pi(\Delta)}=$ $\overline{B r_{1} B}$, we can take $g=r_{1}$ in (5.14). We have $\operatorname{dim} M=\operatorname{dim} \operatorname{ad}(B) V(12121)$ $=\operatorname{dim} \operatorname{ad}(B)\left(\mathbf{C} X_{\alpha_{2}}\right)=\operatorname{dim} \operatorname{ad}(B) X_{\alpha_{2}}=\operatorname{dim} \operatorname{ad}(\mathfrak{b}) X_{\alpha_{2}}=\operatorname{dim}\left(\mathbf{C} X_{\alpha_{2}}+\mathbf{C} X_{\alpha_{1}+\alpha_{2}}+\right.$ $\left.\mathrm{C} X_{3 \alpha_{1}+2 \alpha_{2}}\right)=3<\operatorname{card} R_{+}-1-l\left(r_{1}\right)=4$. Thus (5.14.3) is not satisfied, and hence $\Lambda(12121)$ does not contain the irreducible component $\Delta$ of $\Lambda(121) \cap \Lambda(1)$.

Last let us show the necessary local irreducibility of $\Lambda(w)$ 's, using (5.17). The case where $w=121212$ is trivial. Let $w=12121, Y_{0}=X_{\alpha_{2}}$, $w^{\prime}=121$, and $Y_{1}=X_{\alpha_{2}}+X_{3 \alpha_{1}+2 \alpha_{2}}$. Since $Y_{0}, Y_{1} \in V^{*}(1)$ and they are G(1)equivalent, ad $(G) Y_{0}=\operatorname{ad}(G) Y_{1}$ and (5.16.1) holds. (The weighted Dynkin diagram of this nilpotent class is $0 E 1$.) Since $\mathrm{ad}(\mathrm{g}) \cdot\left(X_{\alpha_{2}}+X_{3 \alpha_{1}+2 \alpha_{2}}\right)$ is spanned by $X_{\gamma}$ for $\gamma=\alpha_{1}+\alpha_{2}, \alpha_{2}, 3 \alpha_{1}+2 \alpha_{2}$ and by $\alpha_{2}^{\vee}+c_{1} X_{3 \alpha_{1}+\alpha_{2}}$, $X_{-\alpha_{1}}+c_{2} X_{2 \alpha_{1}+\alpha_{2}},\left(3 \alpha_{1}+2 \alpha_{2}\right)^{\vee}+c_{3} X_{-3 \alpha_{1}-\alpha_{2}}$ with some $c_{i} \in \mathbf{C}^{\times}$, we get $\operatorname{dim} C$ $=\operatorname{dim}\left(\operatorname{ad}(\mathfrak{g}) Y_{1}\right)=6$ and $\operatorname{dim}\left(\operatorname{ad}(\mathfrak{g}) Y_{1} \cap \mathfrak{n}\right)=3$. Taking the representative $\dot{w}^{\prime}$ of 121 suitably, we may assume that ${ }^{w^{\prime}} Y_{1}=Y_{1}$. Thus (5.17.1) holds. With $\mathfrak{q}=\mathfrak{b}$, (5.17.3) holds. Hence we get the desired irreducibility. (By $[1], Z_{G}\left(Y_{1}\right)$ is known to be connected. Thus we can also get the irreducibility using (5.17.2).) Let $w=121, Y_{0}=X_{\alpha_{1}+\alpha_{2}}, w^{\prime}=1$, and $Y_{1}=X_{\alpha_{2}}+X_{\alpha_{1}+\alpha_{2}}$. (Here we should take a representative of the codimension one orbit of $\left(G(1), V^{*}(1)\right)$ different from the one given in (7.1.1).) As is seen from (7.1.1), (5.16.1) 
holds. (The weighted Dynkin diagram of the nilpotent class of $X_{\alpha_{1}+\alpha_{2}}$ is $1 \notin 0$.) In the present case, we check $(5.17 .1)$ by a direct calculation. Let $\sigma$ be any matrix of the form (4.6.1) whose $i j$-components are zero whenever $i \geq j$. Put

$$
\begin{aligned}
& \Sigma_{1}=\left\{\sigma \mid a_{1}=0,\left(a_{1}^{\prime} a_{4}^{\prime}+a_{2} a_{3}\right)^{2}+4\left(a_{2} a_{4}^{\prime}+a_{3}^{2}\right)\left(a_{1}^{\prime} a_{3}-a_{2}^{2}\right)=0\right\}, \\
& \Sigma_{2}=\left\{\sigma \mid a_{1}^{\prime}=0,-2 a_{1} a_{5}^{\prime}+2 a_{2} a_{4}^{\prime}+\frac{3}{2} a_{3}^{2}=0\right\} .
\end{aligned}
$$

Then $\Sigma_{1}$ and $\Sigma_{2}$ are the irreducible components of $\overline{\operatorname{ad}(G) Y_{1}} \cap \mathrm{n}$. Then both $Y_{1}=X_{\alpha_{2}}+X_{\alpha_{1}+\alpha_{2}}$ and ${ }^{w^{\prime}} Y_{1}=X_{2 \alpha_{1}+\alpha_{2}}+X_{3 \alpha_{1}+\alpha_{2}}$ lie in the non-singular locus of $\Sigma_{1}$ and not in $\Sigma_{2}$. Hence (5.17.1) holds. With $q=\left\langle\mathrm{t}, X_{-\alpha_{1}}, X_{-3 \alpha_{1}-2 \alpha_{2}}\right\rangle$ (linear span), (5.17.3) holds. (In the present case, $\operatorname{dim} C=8$. Since $Z_{G}\left(Y_{1}\right)$ is connected [1], (5.17.2) is also satisfied.) Thus the local $b$-function at $\Lambda(1)$ is

$$
b_{2}(s)=(s+1)\left(s+\frac{3}{2}\right)\left(s+\frac{4}{3}\right)\left(s+\frac{5}{3}\right) \text {. }
$$

Remark 7.1.5. We can show that all the $\Lambda(w)$ 's in the above diagram are contained in $\mathbf{W}$ also in the following way. Assume for example that $\mathbf{W}$ does not contain $\Lambda(1)$. Then by $[\mathbf{2 4}, 7.1]$ (with an obvious modification suitable for the present situation), $\mathscr{D} f^{\lambda_{2} \omega_{2}+0}$ does not have a proper coherent $\mathscr{D}$-submodule, if $b\left(\lambda_{2}-j\right) \neq 0$ for any $j \in \mathbf{Z}$, where $b(s)$ is a divisor of $(s+1)\left(s+\frac{4}{3}\right)\left(s+\frac{5}{3}\right)$. Then, for a sufficiently large integer $m$,

$$
\begin{aligned}
H_{B w_{S} P}^{0}\left(\mathscr{D}_{G} f^{\lambda_{2} \varpi_{2}+0}\right) & =\mathscr{D}_{G}\left(f^{\lambda_{2} \varpi_{2}+0}\right)\left[f_{2}^{-1}\right] & & \text { by }(2.6) \\
& =\mathscr{D}_{G}\left(f^{\left(\lambda_{2}-m\right) \omega_{2}+0}\right) & & \text { by }(1.4)
\end{aligned}
$$

Hence by $[8,9.4], M\left(\lambda_{2} \varpi_{2}, \mathfrak{p}\left(\left\{r_{1}\right\}\right)\right)$ is irreducible if $\lambda_{2} \notin \frac{1}{3} \mathbf{Z}$. But by the Jantzen's criterion [12], we can show that $M\left(s \varpi_{2}, \mathfrak{p}\left(\left\{r_{1}\right\}\right)\right)$ is irreducible if and only if $s \notin\left\{-1+j,-\frac{3}{2}+j,-\frac{4}{3}+j,-\frac{5}{3}+j \mid j=1,2, \cdots\right\}$. Thus a contradiction arises. In the same way we can show that a contradiction arises if we assume some of $\Lambda(w)$ 's is not contained in W. It is interesting that we can get information about the explicit form of the $b$-function by the representation theoretic argument.

Remark 7.2. We can determine the holonomy diagrams for $(\mathfrak{g}, I)$ for rank $\mathfrak{g} \leq 2$ and $\operatorname{card} I=1$. 

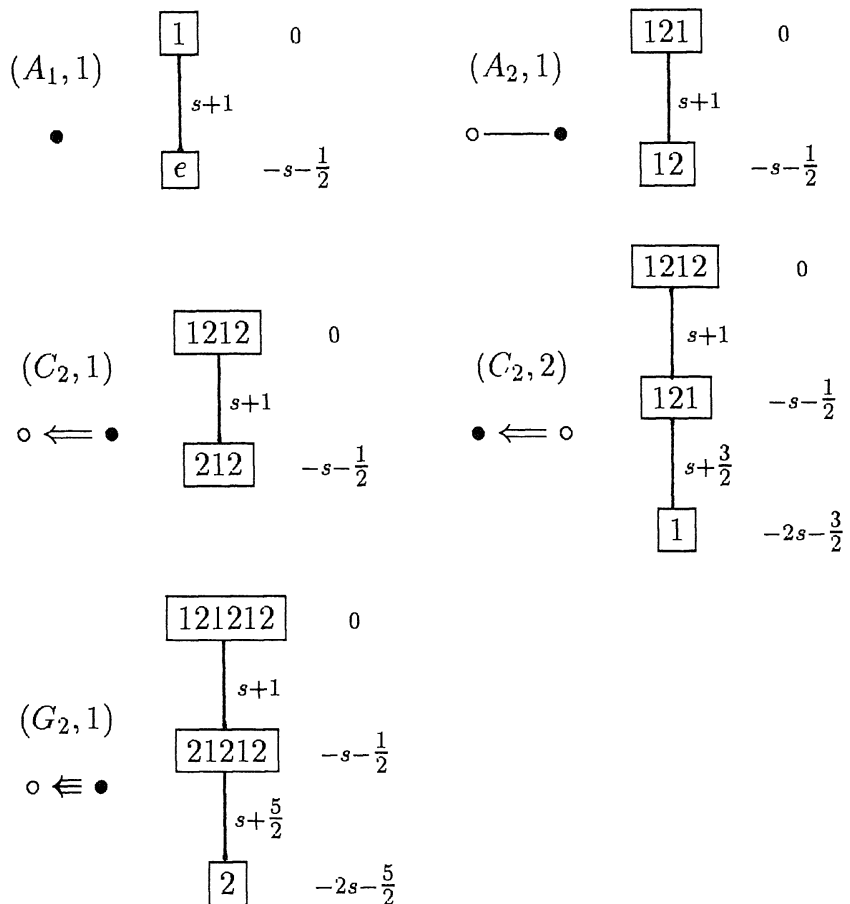

(Figure 4)

See (7.1) for $\left(G_{2}, 2\right)$.

Remark 7.3. For $w \in W$, let $\mathscr{L}_{w^{\prime}}=\mathscr{Z}_{X} \otimes_{U(\mathrm{~g})} L_{w}$ as in (6.4). In the case $\left(G_{2}, 2\right)$, we can show that

$$
\mathbf{C h}\left(\mathscr{D}_{G}[s] f_{2}^{s} / s_{\mathscr{D}_{G}}[s] f_{2}^{s}\right)=\mathbf{C h}\left(p_{X}^{*} \mathscr{L}_{121212}\right)+\mathbf{C h}\left(p_{X}^{*} \mathscr{L}_{12121}\right)
$$

by (6.5.4). Since $\mathscr{L}_{121212}=\mathfrak{C}_{X}, \mathbf{C h}\left(p_{X}^{*} \mathscr{L}_{121212}\right)=[\Lambda(121212)]$. Since $\Lambda(w)$ $\left(w \in W_{0}(I)\right)$ are all good Lagrangian varieties in the case $\left(G_{2}, 2\right)$, the left hand side of $(7.3 .1)$ is equal to $[\Lambda(121212)]+[\Lambda(12121)]+[\Lambda(121)]+[\Lambda(1)]$ by $[24,4.8]$. Hence

$$
\mathbf{C h}\left(p_{X}^{*} \mathscr{L}_{12121}\right)=[\Lambda(12121)]+[\Lambda(121)]+[\Lambda(1)] .
$$

Especially, the characteristic variety of $\mathscr{L}_{12121}$ is not irreducible. This phenomenon can be also explained as follows. Generally, assume that good Lagrangians $\Lambda(w)$ and $\Lambda\left(w^{\prime}\right)$ have an intersection of codimension one, $\operatorname{ord}_{\Lambda(w)} f^{s}=-\mathrm{ms}-\frac{\mu}{2}, \quad \operatorname{ord}_{\Lambda\left(w^{\prime}\right)} f^{s}=-m^{\prime} s-\frac{\mu^{\prime}}{2}, \quad$ and $m^{\prime}>m$. Then in a neighbourhood of a generic point of $\Lambda(w) \cap \Lambda\left(w^{\prime}\right), \mathscr{E}[s] f^{s} /(s-x) \mathscr{E}[s] f^{s}$ does not have a proper coherent $\mathscr{E}$-submodule if the polynomial $b_{\Lambda\left(w^{\prime}\right)}(s) / b_{\Lambda\left(w^{\prime}\right)}(s)$ does not vanish at $\alpha+j$ for any $j \in \mathbf{Z}[\mathbf{2 4}, 7.1]$. In the present case, since the 
characteristic variety of $p_{X}^{*} \mathscr{L}_{12121}$ contains $\Lambda(12121)$, it also contains $\Lambda(121)$ and $1(1)$. Such a phenomenon was observed by Kashiwara-Tanisaki [17, 5.4, Example].

\section{4. $\left(A_{l}, 1, l\right) \quad(l \geq 3) \underset{\alpha_{1}}{\bigcirc}-\cdots-\underset{\alpha_{2}}{\bigcirc}-\cdots$}

Let $\mathfrak{g}$ be the Lie algebra of type $A_{l}(l \geq 3)$ and $I=S \backslash\{1, l\}$. Then $I^{\prime}=I$ and

$$
\left(W_{I^{\prime}} \backslash W / W_{I}\right)_{l}=\left\{w_{I}, w_{I \cup\{1\}}, w_{I \cup\{l\}}, w_{S} r_{1} r_{l}, w_{S} r_{1}, w_{S} r_{l}, w_{S}\right\} .
$$

We give the sets $R(w)$ and $R^{\prime}(w)$ in the following table.

$\begin{array}{llll}w_{I} & R_{+} \backslash R_{I} & R_{I,+} \cup R_{I,-}\left(=R_{I}\right) & 2 l-1 \\ w_{I \cup\{1\}} & R_{+} \backslash R_{I \cup\{1\}} & R_{I_{1},+} \cup R_{I,-} & \\ w_{I \cup\{l\}} & R_{+} \backslash R_{I \cup\{l\}} & R_{I_{2},+} \cup R_{I,-} \\ w_{S} r_{1} r_{l} & \left\{\alpha_{1}, \alpha_{l}\right\} & r_{1} r_{l} R_{I,+} \cup R_{I,-} & 2 \\ w_{S} r_{1} & \left\{\alpha_{1}\right\} & r_{1} R_{I,+} \cup R_{I,-} & 1 \\ w_{S} r_{l} & \left\{\alpha_{l}\right\} & r_{l} R_{I,+} \cup R_{I,-} & 1 \\ w_{S} & \phi & R_{I,+} \cup R_{I,-}\left(=R_{I}\right) & 0\end{array}$

Here $I_{1}=\{1,2, \cdots, l-2\}$ and $I_{2}=\{3,4, \cdots, l\}$. The contents are from the left $w, R(w)=w^{-1} R_{+} \cap R_{+}, R^{\prime}(w)=w^{-1} R_{I^{\prime},-} \cup R_{I^{-}-}$and card $R(w)$. We have left blank the last column for $w=w_{I \cup\{1\}}$ and $w_{I \cup\{l\}}$ since we do not need them. The orbit structure of the colocalization $\left(G(w), V^{*}(w)\right)$ is partly given in the following table.

$\begin{array}{llll}w_{I} & X_{12}+X_{2, l+1} & X_{12}+X_{3 . l+1} & \alpha_{1} \\ w_{I \cup\{1\}} & X_{2, l+1} & X_{14}+X_{34}(l=3) & \alpha_{2}+\alpha_{3}(l=3) \\ w_{I \cup\{l\}} & X_{13} & X_{12}+X_{14}(l=3) & \alpha_{1}+\alpha_{2}(l=3) \\ w_{S} r_{1} r_{l} & X_{12}+X_{l, l+1} & X_{12} & \alpha_{l} \\ & & X_{l . l+1} & \alpha_{1} \\ w_{S} r_{1} & X_{12} & 0 & \alpha_{1} \\ w_{S} r_{l} & X_{l . l+1} & 0 & \alpha_{l} \\ w_{S} & 0 & & \end{array}$

Here we realize $G$ as $S L_{l+1}(\mathbf{C}), X_{i j}$ denotes the matrix whose $i j$-component is 1 and others are 0 , and $\varepsilon_{i}$ denotes the character of $t$ defined by $\operatorname{diag}\left(t_{1}, \cdots, t_{l+1}\right) \rightarrow t_{i}$. The meaning of this table is the same as (7.1.1). Codimension one orbits do not exist for $w=w_{I \cup\{1\}}$ or $w=w_{I \cup\{l\}}$ unless 
$l=3$. There are two codimension one orbits for $w=w_{S} r_{1} r_{l}$. Let $Y_{0}$ be the element in the second column for $w$, and find an element $A_{0}$ in $\mathrm{t}$ such that ad $\left(A_{0}\right) Y_{0}=Y_{0}$. By (5.5),

$$
\begin{aligned}
\operatorname{ord}_{\Lambda(w)} f_{1}^{\lambda_{1}} f_{l}^{\lambda_{l}}= & -\lambda_{1}\left\langle w^{-1} \varpi_{l}+\varpi_{1}, A_{0}\right\rangle-\lambda_{l}\left\langle w^{-1} \varpi_{1}+\varpi_{l}, A_{0}\right\rangle \\
& -\sum_{\alpha \in R(w)}\left\langle\alpha, A_{0}\right\rangle+\frac{1}{2} \operatorname{card} R(w) .
\end{aligned}
$$

The characters $w^{-1} \varpi_{l}+\varpi_{1}, w^{-1} \varpi_{1}+\varpi_{l}, \sum_{\alpha \in R(w)} \alpha$ and the order of $f_{1}^{s_{1}} f_{l}^{s_{l}}$ at $\Lambda(w)$ are given in the following table from the left in this order, where the most left column gives $w$.

$$
\begin{array}{lllll}
w_{I} & \varepsilon_{1}-\varepsilon_{l+1} & \varepsilon_{1}-\varepsilon_{l+1} & l\left(\varepsilon_{1}-\varepsilon_{l+1}\right) & -2 s_{1}-2 s_{l}-\frac{2 l+1}{2} \\
w_{I \cup\{1\}} & \varepsilon_{1}-\varepsilon_{l+1} & \varepsilon_{l}-\varepsilon_{l+1} & & \\
w_{I \cup\{l\}} & \varepsilon_{1}-\varepsilon_{2} & \varepsilon_{1}-\varepsilon_{l+1} & \\
w_{S} r_{1} r_{l} & \varepsilon_{1}-\varepsilon_{2} & \varepsilon_{l}-\varepsilon_{l+1} & \left(\varepsilon_{1}-\varepsilon_{2}\right)+\left(\varepsilon_{l}-\varepsilon_{l+1}\right) & -s_{1}-s_{2}-1 \\
w_{S} r_{1} & \varepsilon_{1}-\varepsilon_{2} & 0 & \varepsilon_{1}-\varepsilon_{2} & -s_{1}-\frac{1}{2} \\
& & & & -s_{l}-\frac{1}{2} \\
w_{S} r_{l} & 0 & \varepsilon_{l}-\varepsilon_{l+1} & \varepsilon_{l}-\varepsilon_{l+1} & 0 \\
w_{S} & 0 & 0 & 0 &
\end{array}
$$

We have left blank the last two columns for $w=w_{I \cup\{1\}}$ and $w_{I \cup\{l\}}$, since we do not need them. In fact, as in (7.1), we can show that $\left\langle w^{-1} \varpi_{l}+\varpi_{1}, A_{0}\right\rangle$ really depends on the choice of $A_{0}$ for these $w$. Hence $w_{I \cup\{1\}}, w_{I \cup\{l\}} \notin W_{0}(I)$. (Cf. (5.5.2).)

Let us find elements in $\Lambda\left(w_{I}\right) \cap \Lambda\left(w_{S} r_{1} r_{l}\right)$ by using (5.10). As is easily seen, $\left(G\left(w_{I}\right), V\left(w_{I}\right)\right)$ has 6 orbits represented by $X_{\alpha_{1}}+X_{\alpha_{2}+\cdots+\alpha_{l}}(\operatorname{dim}=2 l-1)$, $X_{\alpha_{1}}+X_{\alpha_{l}}(\operatorname{dim}=2 l-2), X_{\alpha_{1}}(\operatorname{dim}=l), X_{\alpha_{l}}(\operatorname{dim}=l), X_{\alpha_{1}+\cdots+\alpha_{l}}(\operatorname{dim}=1)$ and 0 . Here we calculate the case $l=5$, since it has an enough general feature. In this case $w_{S} r_{1} r_{l}=r_{1} w_{S} r_{1}=21.321 .4321 .5432$, and $w_{I}=2.32 .432$. Put $w_{i}=$ $21.321 .4321 .54 \cdots(i+2) \quad(0 \leq i \leq 3), \quad w_{4}=21.321 .4321, \quad w_{5}=21.321 .432, \quad w_{6}=$ 21.32.432 and $w_{7}=2.32 .432$. Then $\gamma_{i}=\alpha_{i+2}(0 \leq i \leq 3)$ and $\gamma_{3+i}=\alpha_{1}+\cdots+\alpha_{i}$ $(1 \leq i \leq 3)$. Hence $E_{0}=\left\{\alpha_{1}, \alpha_{5}\right\}, E_{i+1}=\left\{\alpha_{1}+\cdots+\alpha_{i+2}, \alpha_{5}\right\} \quad(0 \leq i \leq 1=l-4)$, $E_{3}\left(=E_{l-2}\right)=\left\{\alpha_{1}+\cdots+\alpha_{4}, \alpha_{4}+\alpha_{5}\right\}, \quad E_{4}=\left\{\alpha_{1}+\cdots+\alpha_{5}, \alpha_{4}+\alpha_{5}\right\}=E_{5}=E_{6}$ $=E_{7}\left(=E_{2 l-3}\right)$. In this way we can show in general that $X_{\alpha_{l-1}+\alpha_{l}} \in w^{\prime-1}(\Lambda(w)$ $\left.\cap \Lambda\left(w^{\prime}\right)\right)$, where $w=w_{S} r_{1} r_{l}$ and $w^{\prime}=w_{I}$. As is easily seen, $X_{\alpha_{l-1}+\alpha_{l}}$ and $X_{\alpha_{l}}$ belong to the same $G\left(w^{\prime}\right)$-orbit, and hence $\mathfrak{g}\left(\alpha_{l}\right)+\mathfrak{g}\left(\alpha_{1}+\cdots+\alpha_{l}\right)\left(\subset \overline{G\left(w^{\prime}\right) X_{\alpha_{l}}}\right)$ is contained in the above intersection. Considering the automorphism of the Dynkin diagram, we can show that $g\left(\alpha_{1}\right)+g\left(\alpha_{1}+\cdots+\alpha_{l}\right)$ is also contained in 


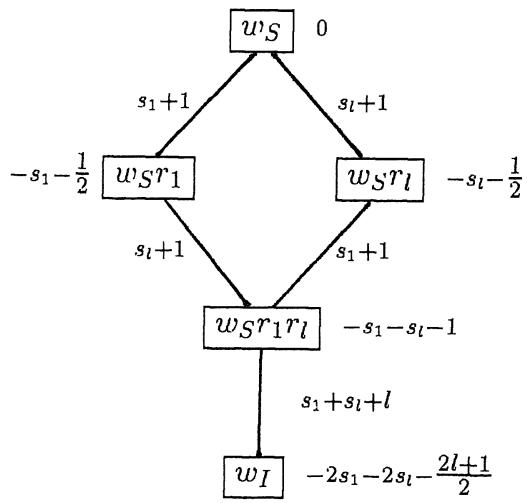

(Figure 5)

the same intersection. By (5.12), $\operatorname{Grass}_{2}\left(w^{\prime-1}\left(\Lambda(w) \cap \Lambda\left(w^{\prime}\right)\right)\right)$ contains a $G\left(w^{\prime}\right)$ stable connected set, say $Z$, containing $\mathfrak{g}\left(\alpha_{i}\right)+\mathfrak{g}\left(\alpha_{1}+\cdots+\alpha_{l}\right)(i=1, l)$. Because of the orbit structure of $\left(G\left(w^{\prime}\right), V^{*}\left(w^{\prime}\right)\right), \cup Z$ should contain the orbit of codimension one. Hence $\Lambda(w)$ and $\Lambda\left(w^{\prime}\right)$ intersect in codimension one.

By (5.8.1), we get edges $w_{S}-w_{S} r_{1}-w_{S} r_{1} r_{l}$ and $w_{S}-w_{S} r_{l}-w_{S} r_{1} r_{l}$. A direct calculation shows that all the codimension one intersections obtained above have intersection exponents $(1: 0)$, if we assume the necessary local irreducibility, whose proof we shall give afterwards. Thus we get a holonomy diagram. Let us show that the above diagram is the holonomy diagram. By (5.7), we can show that $W_{0}(I)=\left\{w_{S}, w_{S} r_{1}, w_{S} r_{l}, w_{S} r_{1} r_{l}, w_{I}\right\}$. Obviously, the edges $w_{S}-w_{S} r_{1} r_{l}$ and $w_{S}-w_{I}$ are not contained in the holonomy diagram. Considering the automorphism of the Dynkin diagram, it remains to show that the edge $w_{S} r_{1}-w_{I}$ is not contained in the holonomy diagram. Since the semi-invariant $f_{1}^{\prime}$ is given by $f_{1}^{\prime}(x)=x_{11}\left(x=\left(x_{p q}\right) \in S L_{l+1}\right), \overline{B w_{s} r_{1} B}$ is a non-singular hypersurface of $G$ (cf. $(2.6,(1))$. Thus the fibre of $\Lambda\left(w_{s} r_{1}\right)$ at any point is a one dimensional vector space. Since the codimension of $\overline{B w_{I} B}=P(I)$ in $G$ is greater than one, $\operatorname{dim}\left(\Lambda\left(w_{S} r_{1}\right) \cap \Lambda\left(w_{I}\right)\right)<\operatorname{dim} G-1$, i.e., the edge $w_{S} r_{1}-w_{I}$ is not contained in the holonomy diagram.

Last let us show the necessary local irreducibility of $\Lambda(w)$ 's, using (5.17). Let $w=w_{S} r_{1} r_{l}$ and $w^{\prime}=w_{I}$. (The remaining cases are obvious from (5.8.1).) Since we can take $Y_{0}=Y_{1}=X_{12}+X_{l, l+1}$, (5.16.1) holds. Let us check $(5.17 .1)_{z}$. Since $\mathrm{ad}(\mathrm{g}) \cdot\left(X_{12}+X_{l, l+1}\right)$ is spanned by $X_{i 2}(i \neq 2, l+1)$, $X_{i, l+1}(\mathrm{i} \neq 2, l+1), X_{1 j}(j \neq 1, l), X_{l j}(j \neq 1, l), X_{11}-X_{22}, X_{1 l}-X_{2, l+1}, X_{l 1}-$ $X_{l+1,2}$, and $X_{l l}-X_{l+1, l+1}$, we $\operatorname{get} \operatorname{dim} C=\operatorname{dim}\left(\operatorname{ad}(\mathrm{g}) Y_{1}\right)=4 l-4$ and $\operatorname{dim}\left(\operatorname{ad}(\mathfrak{g}) Y_{1} \cap \mathfrak{n}\right)=2 l-2$. Thus we get $(5.17 .1)_{e}$. Since

$$
\begin{gathered}
\Omega=\left\{\left(x_{i j}\right) \mid \sum_{i=2}^{l} x_{1 i} x_{i, l+1}=0,\left(x_{12}, x_{13}, \cdots, x_{1 l}\right) \neq(0, \cdots, 0),\right. \\
\left.\left(x_{2, l+1}, x_{3, l+1}, \cdots, x_{l, l+1}\right) \neq(0, \cdots, 0)\right\},
\end{gathered}
$$


${ }^{w^{\prime}} \Omega=\Omega$, and hence we also get $(5.17 .1)_{w^{\prime}}$. Thus the local $b$-functions at $\Lambda\left(w_{I}\right)$ are given by

$$
\begin{aligned}
& b_{1}\left(s_{1}, s_{l}\right)=\left(s_{1}+1\right)\left(s_{1}+s_{l}+l\right), \\
& b_{l}\left(s_{1}, s_{l}\right)=\left(s_{l}+1\right)\left(s_{1}+s_{l}+l\right) .
\end{aligned}
$$

7.5. $\left(A_{3}, 1,2\right) \quad \bigcirc_{\alpha_{1}}-\underset{\alpha_{2}}{\bigcirc}-\underset{\alpha_{3}}{\bullet}$

Let $g$ be the Lie algebra of type $A_{3}$ and $I=\{3\}$. Then $I^{\prime}=\{1\}$ and

$$
\left(W_{I^{\prime}} \backslash W / W_{I}\right)_{l}=\{121321,12321,12132,1232,1213,123,13\}=W_{0}(I) .
$$

The holonomy diagram is

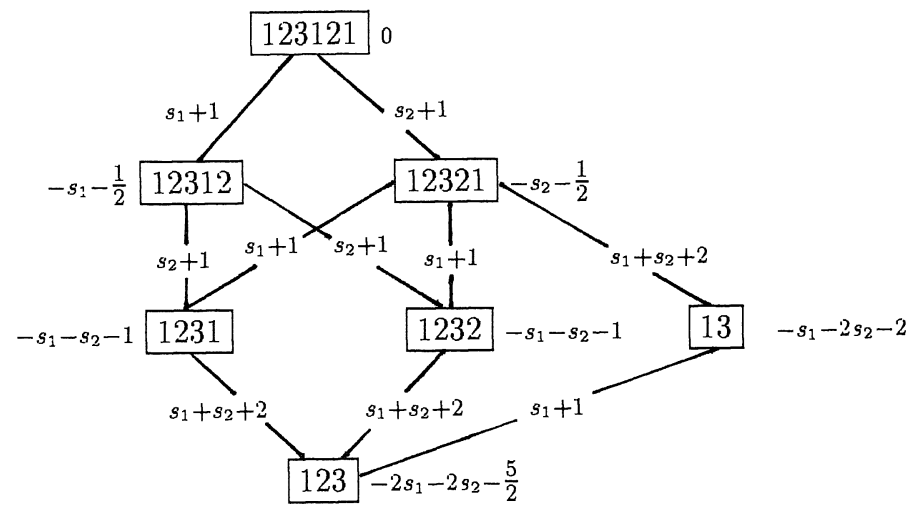

(Figure 6)

The local $b$-functions at $\Lambda(123)$ are given by

$$
\begin{aligned}
& b_{1}\left(s_{1}, s_{2}\right)=\left(s_{1}+1\right)\left(s_{1}+s_{2}+2\right), \\
& b_{2}\left(s_{1}, s_{2}\right)=\left(s_{2}+1\right)\left(s_{1}+s_{2}+2\right) .
\end{aligned}
$$

7.6. If $(\mathfrak{g}, \mathfrak{p})$ is the complexification of a real simple Lie algebra of (real) rank one and its minimal parabolic subalgebra, then $(\mathfrak{g}, I)$ is given by one of the following diagrams.

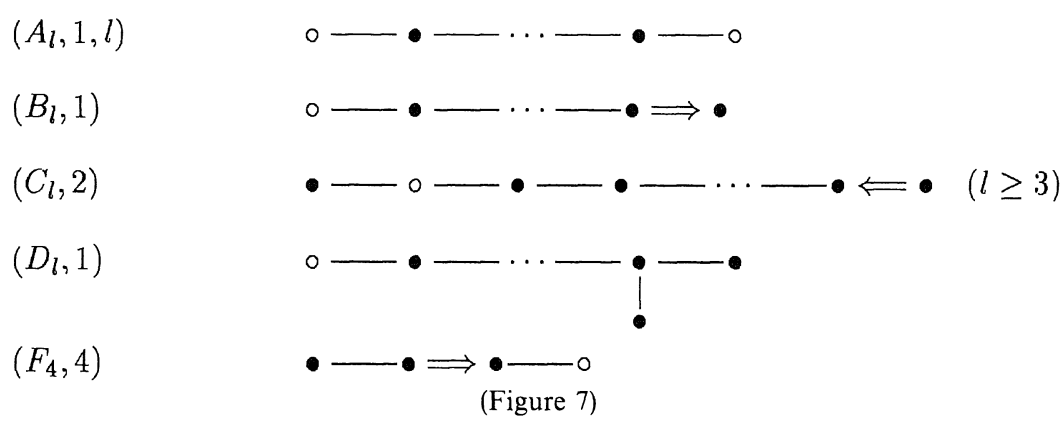



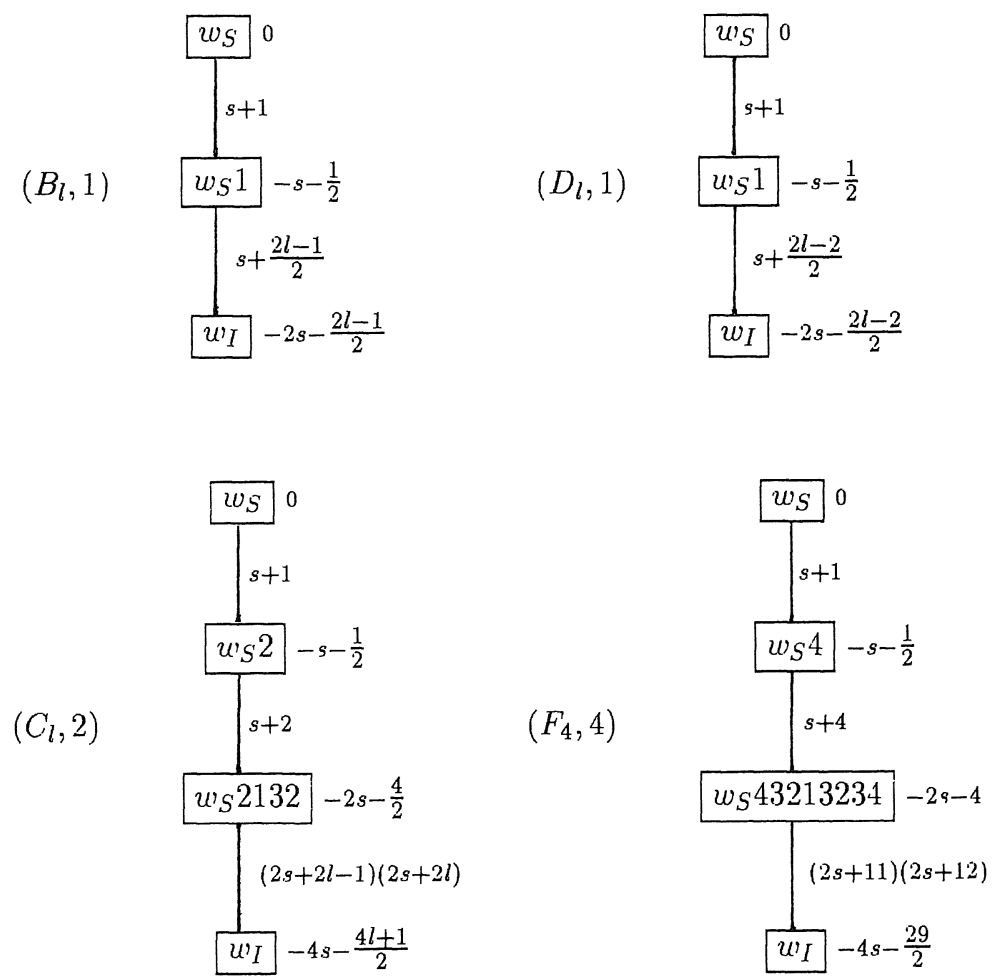

(Figure 8)

The holonomy diagrams are given in Figure 8.

See (7.4) for $\left(A_{l}, 1, l\right)$. In these cases, $\Lambda\left(w_{I}\right)$ appears at the bottom of the holonomy diagram. The local $b$-function there is the product of all the factors attached to the edges.

\section{§8. Examples (3)}

8.0. In this section, we give examples which need calculation more complicated than those of Section 7. We start with a slight improvement of our algorithm.

Lemma 8.1. Let $I, J \subset S, \lambda_{J}=\sum_{i \in S-J} \varpi_{i}$, and $\Phi(J, I)=\left\{\mu \in W \lambda_{J} \mid\left\langle\mu, \beta^{\vee}\right\rangle\right.$ $\leq 0$ for any $\left.\beta \in \Pi_{I}\right\}$. (1) The mapping $\varphi=\varphi_{J}: w \rightarrow w^{-1} \lambda_{J}$ gives a bijection $\left(W_{J} \backslash W / W_{I}\right)_{l} \stackrel{\sim}{\rightarrow} \Phi(J, I)$. Let $w \in\left(W_{J} \backslash W / W_{I}\right)_{l} . \quad$ (2) $\quad w^{-1} R_{J,-}=\left\{\alpha \in R_{+} i\left\langle w^{-1} \lambda_{J}\right.\right.$, $\left.\left.\alpha^{v}\right\rangle=0\right\}$. (3) $R(w)=w^{-1} R_{+} \cap R_{+}=\left\{\alpha \in R_{+} \mid\left\langle w^{-1} \lambda_{J}, \alpha^{v}\right\rangle>0\right\}$.

Proof. (1) . Let $w \in\left(W_{J} \backslash W / W_{I}\right)_{l}, \beta \in \Pi_{I}$ and $w=w^{\prime} r_{\beta}$. Then $\left\langle w^{-1} \lambda_{J}, \beta^{\vee}\right\rangle$ $=-\left\langle\lambda_{J}, w^{\prime} \beta^{\vee}\right\rangle \leq 0$. Hence $\varphi$ is well-defined. The isotropy subgroup, say 
$Z$, of $W$ at $\lambda_{J}$ is generated by reflections with respect to $\alpha \in R$ such that $\left\langle\lambda_{J}, \alpha^{\vee}\right\rangle=0$, i.e., $\alpha \in R_{J}$. Hence $Z=W_{J}$. Thus $\left(W_{J} \backslash W\right)_{l} \stackrel{\sim}{\rightarrow} W \lambda_{J}$, whose restriction $\varphi$ is injective. Let $w \in\left(W_{J} \backslash W\right)_{l}$ and $w^{-1} \lambda_{J} \in \Phi(J, I)$. It suffices to prove that $w \in\left(W_{J} \backslash W / W_{I}\right)_{l}$. Put $K=\left\{r_{\beta} \in I \mid\left\langle w^{-1} \lambda_{J}, \beta^{v}\right\rangle=0\right\}$. Take $w^{\prime} \in$ $\left(W / W_{K}\right)_{l}$ so that $w^{\prime} W_{K}=w W_{K}$. Then $w^{\prime} \geq w, w^{-1} \lambda_{J}=w^{\prime-1} \lambda_{J}$, and hence $W_{J} w=W_{J} w^{\prime}$. Since $w \in\left(W_{J} \backslash W\right)_{l}, w \geq w^{\prime}$. Thus $w=w^{\prime} \in\left(W / W_{K}\right)_{l}$. For $r_{\beta} \in$ $I \backslash K \quad(\beta>0)$, we have $\left\langle\lambda_{J}, w \beta^{\vee}\right\rangle=\left\langle w^{-1} \lambda_{J}, \beta^{\vee}\right\rangle<0, w \beta^{\vee}<0$, and hence $w r_{\beta}<w$. Thus $w \in\left(W / W_{I}\right)_{l} \cap\left(W_{J} \backslash W\right)_{l}=\left(W_{J} \backslash W / W_{I}\right)_{l}$, and we get the surjectivity of $\varphi$. (2) If $\alpha>0$ and $0=\left\langle w^{-1} \lambda_{J}, \alpha^{v}\right\rangle=\left\langle\lambda_{J}, w \alpha^{v}\right\rangle$, then $w \alpha \in R_{J,-}$. The converse is similar. (3) Let $\alpha>0$. If $\left\langle\lambda_{J}, w \alpha^{\vee}\right\rangle>0$ (resp. $<0$ ), then $w \alpha^{v}>0$ (resp. <0). Together with $(2)$, we get $(3)$.

Remark 8.1.1. In (8.1), we may replace $\lambda_{J}$ with any $\sum_{i \in S-J} l_{i} \varpi_{i}$ such that every $l_{i}$ is positive.

8.2. Here we indicate how to calculate the inverse of $\varphi$ in (8.1). For the sake of simpicity, we consider only the type $A_{l}$. Represent an element $\mu=\sum \mu_{j} \varpi_{j}$ by attaching $\mu_{j}$ to the $j$-th vertex of the Dynkin diagram. Apply $r_{i}$ if $i$-th label is negative, noting that $r_{i}\left(\sum_{j} \mu_{j} \varpi_{j}\right)=\sum_{|j-1|>1} \mu_{j} \varpi_{j}+\left(\mu_{i-1}+\mu_{i}\right) \varpi_{i-1}$ $-\mu_{i} \varpi_{i}+\left(\mu_{i+1}+\mu_{i}\right) \varpi_{i+1}$. (Here $\varpi_{0}=\varpi_{l+1}=0$.) Repeat this until no negative labels remain. Let $\lambda_{I}=\sum \lambda_{i} \varpi_{i}$ be the element given by the final diagram. For example, let us calculate $\varphi^{-1}(\mu)$ for $\mu=\varpi_{1}-2 \varpi_{2}$ :

$$
\mu=1 \overline{2} 0 \stackrel{2}{\longrightarrow} \overline{1} 2 \overline{2} \stackrel{1}{\longrightarrow} 11 \overline{2} \stackrel{3}{\longrightarrow} 1 \overline{1} 2 \stackrel{2}{\longrightarrow} 011=\lambda_{I}, \quad I=\{1\} .
$$

Here $\bar{n}$ denotes $-n$. The product of $r_{i}$ 's in this order (e.g., 2132 in this example) gives the shortest $w^{\prime}$ such that $w^{\prime} \lambda_{I}=\mu$. Hence $\varphi_{I}^{-1}(\mu)=\left(w^{\prime} w_{I}\right)^{-1}$.

8.3. Put $V(w)=\sum_{\alpha \in R(w)} \mathfrak{g}(-\alpha)$. A root $-\alpha$ belongs to $R \backslash\left(w^{-1}\left(R_{+} \cup R_{I^{\prime}}\right)\right.$ $\left.\cup\left(R_{+} \cup R_{I}\right)\right)=w^{-1}\left(R_{-} \backslash R_{I^{\prime}}\right) \cap\left(R_{-} \backslash R_{I}\right)$ if and only if

$$
\alpha>0, w \alpha>0, \alpha \notin R_{I}, \text { and } w \alpha \notin R_{I} .
$$

Let $w \in\left(W_{I^{\prime}} \backslash W / W_{I}\right)_{l}$. If $\alpha \in R_{I}$ or $w \alpha \in R_{I^{\prime}}$, then exactly one of $\alpha$ and $w \alpha$ is positive. Hence (8.3.1) is equivalent to say that $\alpha>0$ and $w \alpha>0$. Thus $\mathfrak{g}=V(w) \oplus\left(\mathfrak{p}^{\prime w}+\mathfrak{p}\right)$. Put $\delta=\sum_{i \in S-I} \varpi_{i}$ and $f=f^{\delta}$. Define the function $f_{w}$ on $\mathfrak{g}$ by $f_{w}(X)=f(w \exp X)$. Since $V^{*}(w)=\left(\mathfrak{p}^{\prime w}+\mathfrak{p}\right)^{\perp}, V^{*}(w)$ is the dual space of $V(w)$. Hence the cotangent bundle of $V(w)$ can be identified with $V(w) \times V^{*}(w)$, in which $\mathbf{W}\left(f_{w^{\prime}} \mid V(w)\right)$ is contained.

Lemma 8.4. $([24,6.9]) \quad \Lambda(w) \subset \mathbf{W}(f)$ if and only if $0 \times V^{*}(w) \subset$ $\mathbf{W}\left(f_{w} \mid V(w)\right)$.

Proof. Let $\mathfrak{p}^{\prime \prime}$ be a subspace of $\mathfrak{p}^{\prime}$ such that $\mathfrak{g}=V(w) \oplus \mathfrak{p}^{\prime \prime w} \oplus \mathfrak{p}$. Put $x=\exp \left(A^{\prime \prime}\right) w \exp (X) \exp (A)$ for $A^{\prime \prime} \in \mathfrak{p}^{\prime \prime}, \quad X \in V(w)$ and $A \in \mathfrak{p}$. Then 
$\left(x, \varepsilon \operatorname{grad}_{A^{\prime \prime}, X, A} \log f(x)\right)=\left(x, \varepsilon \operatorname{grad}_{A^{\prime \prime}, X, A}\left(\left(w_{S} \lambda\right)\left(A^{\prime \prime}\right)+\log f_{w}(X)+\lambda(A)\right)\right)=(x$, $\left.\varepsilon\left(w_{S} \lambda, \operatorname{grad} \log f_{w}(X), \lambda\right)\right)$ for $\varepsilon \in \mathbf{C}$. Here we write $(\mathfrak{g}, X)$ for $g X \in T^{*} G$ (cf. (5.1)). Hence the set of the limits of above points when $\varepsilon, A^{\prime \prime}, X, A \rightarrow 0$ is equal to the set of the limits of $\left(w,\left(0, \varepsilon \operatorname{grad} \log f_{w}(X), 0\right)\right)$ when $\varepsilon, X \rightarrow 0$. Since $\Lambda(w) \subset$ $\mathbf{W}(f)$ if and only if $w V^{*}(w) \subset \mathbf{W}(f)$, we get the assertion.

\section{5. $\left(C_{l}, i\right)$}

Using [12], we can show that the generalized Verma module $M\left(\lambda \varpi_{i}\right.$, $\left.\mathfrak{p}\left(S-\left\{r_{i}\right\}\right)\right)$ is reducible if and only if the following condition is satisfied: (1) If $i=1$, then $\lambda \in\{0,1,2, \cdots\}$. (2) If $i \in 2 \mathbb{Z}$ and $2 l \geq 3 i$, then $\lambda \in\left\{-\frac{1}{2} \times\right.$ $\left.(2 l-i+1)+j,-\frac{1}{2}(2 l-i+2)+j \mid j=1,2, \cdots\right\}$. (3) If $i \in\{3,5,7, \cdots\}$ or $2 l<3 i$, then $\lambda \in\left\{-\frac{1}{2}(2 l-i)+j,-\frac{1}{2}(2 l-i+1)+j \mid j=1,2, \cdots\right\}$. On the other hand, it seems that the $b$-functions are given by the following formula.

$$
b_{i}(s)= \begin{cases}\prod_{v=1}^{i}(s+v) \cdot \prod_{v=3}^{i+2}\left(s+l-i+\frac{v}{2}\right), & \text { if } i \in 2 \mathbf{Z} \text { and } 2 l \geq 3 i \\ \prod_{v=1}^{i}(s+v) \cdot \prod_{v=3}^{i+1}\left(s+l-i+\frac{v}{2}\right), & \text { if } i \in 2 \mathbb{Z}+1 \text { and } 2 l \geq 3 i \\ \prod_{v=1}^{2 l-2 i+1}(s+v) \cdot \prod_{v=3}^{i+1}\left(s+l-i+\frac{v}{2}\right), & \text { otherwise. }\end{cases}
$$

Let us explain how to determine $W_{0}(I)$ and $\operatorname{ord}_{\Lambda(w)} f_{i}^{s}$. See (8.5.3), (8.5.4), (8.5.6), and (8.5.7) below for our result.

In the present case, $I=I^{\prime}=S-\{i\}$. If we realize the root system as in $[3], \Phi\left(I^{\prime}, I\right)($ cf. $(8.1))$ consists of $\varphi(w)=\overbrace{-1, \cdots,-1}^{A}, \overbrace{0, \cdots, 0}^{B}, \overbrace{1, \cdots, 1}^{C},-1 \overbrace{-\cdots,-1}^{D} 1$, $\overbrace{0, \cdots, 0}^{E}$ ) such that $A+B+C=i$ and $B=D$. We sometimes write $\varepsilon(j)$ or simply $j$ for $\varepsilon_{j}$, $[A]$ for $[1, A],[B]$ for $[A+1, A+B]$, etc. We write elements of $[A]$ by $a, a^{\prime}, \cdots$, those of $[B]$ by $b, b^{\prime}, \cdots$, etc. By (8.1),

$$
\begin{aligned}
& R(w)=\left\{b-d, c-d, c-e, b+c, c+c^{\prime}, c+e\right\}, \text { and } \\
& R^{\prime}(w)=\left\{a-a^{\prime}, b-b^{\prime}, c-c^{\prime}, d-d^{\prime}, e-e^{\prime}, a-d, b-e,\right. \\
& a+c, c+d, b+e, b+b^{\prime}, e+e^{\prime}, \\
&\left.-a+b,-a+c,-b+c,-d+e,-d-d^{\prime},-d-e,-e-e^{\prime}\right\} .
\end{aligned}
$$

The action of $G(w)$ on $V^{*}(w)$ is expressed by the following diagram. 

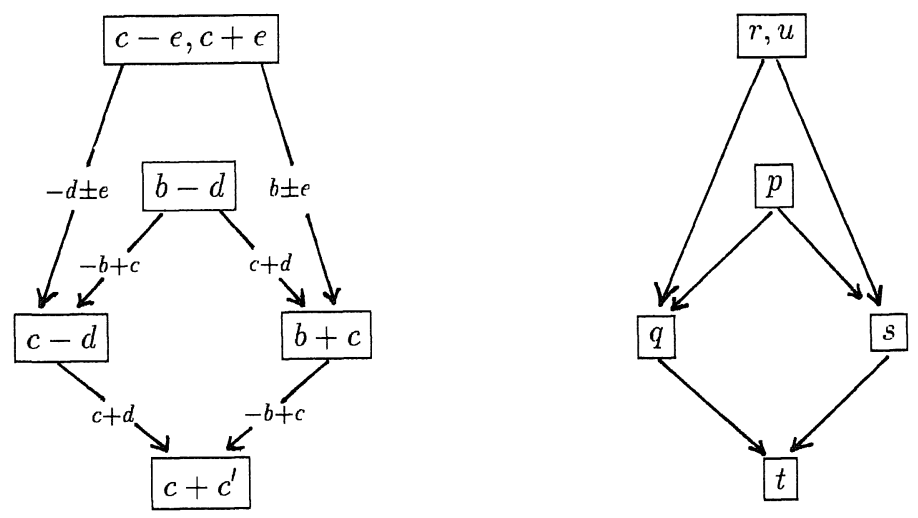

(Figure 9)

For instance, $b-d \stackrel{c+d}{\longrightarrow} b+c$ means ad $(X(c+d)) X(b-d) \in \mathbf{C}^{\times} X(b+c)$. The diagram on the right is the same as the one on the left, but is expressed in terms of matrix, e.g., $p$ means the linear span of the root vectors in $V^{*}(w)$ to which correspond the vectors of the dual basis of $V(w)$ lying in the block $p$ of the matrix (8.5.6) below. Put $Y_{0}^{\prime}=\sum_{b \in[B]} X(\varepsilon(b)-\varepsilon(b+B+C)$ ). Consider the sum $Y_{0}^{\prime \prime}$ of

$$
\begin{aligned}
& X(\varepsilon(A+B+1)-\varepsilon(A+B+C+D+1))+X(\varepsilon(A+B+2)+\varepsilon(A+B+C+D+1)), \\
& X(\varepsilon(A+B+3)-\varepsilon(A+B+C+D+2))+X(\varepsilon(A+B+4)+\varepsilon(A+B+C+D+2)), \cdots .
\end{aligned}
$$

If $C \leq 2 E$ and $C$ is even (resp. odd), then we understand that this sum ends with

$$
\begin{aligned}
& X\left(\varepsilon(A+B+C-1)-\varepsilon\left(A+B+C+D+\frac{C}{2}\right)\right) \\
& +X\left(\varepsilon(A+B+C)+\varepsilon\left(A+B+C+D+\frac{C}{2}\right)\right) \\
& \quad\left(\operatorname{resp.} X\left(\varepsilon(A+B+C)-\varepsilon\left(A+B+C+D+\frac{C+1}{2}\right)\right)\right) .
\end{aligned}
$$

If $C>2 E$, we understand this sum ends with

$$
\begin{aligned}
& X(\varepsilon(A+B+2 E-1)-\varepsilon(A+B+C+D+E)) \\
& \quad+X(\varepsilon(A+B+2 E)+\varepsilon(A+B+C+D+E)) .
\end{aligned}
$$

In the case $C>2 E$, we consider the additional sum $Y_{0}^{\prime \prime \prime}$ of

$$
\begin{aligned}
& X(\varepsilon(A+B+2 E+1)+\varepsilon(A+B+2 E+2)), \\
& X(\varepsilon(A+B+2 E+3)+\varepsilon(A+B+2 E+4)), \cdots .
\end{aligned}
$$

We understand this sum ends with 
$X(\varepsilon(A+B+C-1)+\varepsilon(A+B+C))($ resp. $X(2 \varepsilon(A+B+C)))$, if $C$ is even (resp. odd). Put $Y_{0}=Y_{0}^{\prime}+Y_{0}^{\prime \prime}\left(+Y_{0}^{\prime \prime \prime}\right)$. Then we can show that (8.5.2) $\quad Y_{0}$ belongs to an open orbit of $\left(G(w), V^{*}(w)\right)$.

By (5.5.1), (8.5.1), and (8.5.2),

(8.5.3) $\operatorname{ord}_{\Lambda(w)} f_{i}^{s}$

$$
=\left\{\begin{aligned}
-(B+2 C) s-\frac{1}{2}(B(B+2 C) & \left.+\frac{3}{2} C(C+1)+2(l-i) C\right) \\
& \text { if } C \leq 2 E \text { and } C \text { is even, } \\
-(B+C+2 E) s-\frac{1}{2}\left(B(B+2 C)+\frac{1}{2} C(C+1)+2 E(C+1+2(l-i))\right), & \text { if } C>2 E
\end{aligned}\right.
$$

and, by (5.5.2)

$$
\Lambda(w) \not \subset \mathbf{W} \text { if } C \leq 2 E \text { and } C \text { is odd. }
$$

We realize $s p_{2 n}$ replacing $K=K_{n}$ with the identity matrix $1_{n}$ in (4.5). Then

$$
w=\left(\begin{array}{cccccccccc}
\cdot & \cdot & \cdot & \cdot & \cdot & 1_{A} & \cdot & \cdot & \cdot & \cdot \\
\cdot & \cdot & \cdot & \cdot & \cdot & \cdot & \cdot & \cdot & 1_{B} & \cdot \\
\cdot & \cdot & K_{C} & \cdot & \cdot & \cdot & \cdot & \cdot & \cdot & \cdot \\
\cdot & \cdot & \cdot & \cdot & \cdot & \cdot & 1_{B} & \cdot & \cdot & \cdot \\
\cdot & \cdot & \cdot & \cdot & \cdot & \cdot & \cdot & \cdot & \cdot & 1_{E} \\
-1_{A} & \cdot & \cdot & \cdot & \cdot & \cdot & \cdot & \cdot & \cdot & \cdot \\
\cdot & \cdot & \cdot & -1_{B} & \cdot & \cdot & \cdot & \cdot & \cdot & \cdot \\
\cdot & \cdot & \cdot & \cdot & \cdot & \cdot & \cdot & K_{C} & \cdot & \cdot \\
\cdot & -1_{B} & \cdot & \cdot & \cdot & \cdot & \cdot & \cdot & \cdot & \cdot \\
\cdot & \cdot & \cdot & \cdot & -1_{E} & \cdot & \cdot & \cdot & \cdot & \cdot
\end{array}\right)
$$

and $V(w)$ is the totality of

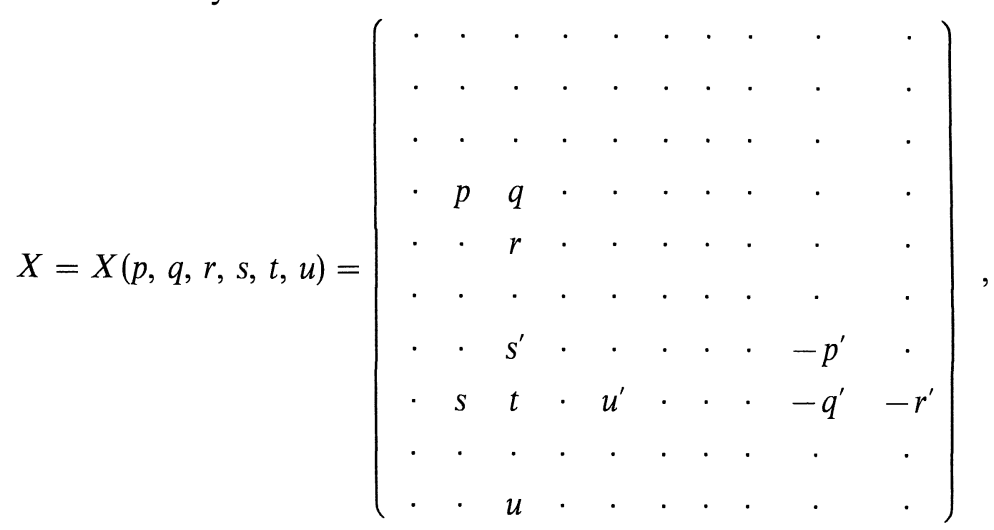


where - stands for zero,' denotes the transposed matrix, $t=t^{\prime}$, and $1 \mathrm{st}$ and 6th blocks from the left (resp. from the top) contain $A$ colums (resp. rows) etc. Then

$$
f_{w}(X(p, q, r, s, t, u)):=(-1)^{A+B+C(C+1) / 2} f(w \exp X)=\left|\begin{array}{cc}
p & q \\
s & t+u^{\prime} r-r^{\prime} u
\end{array}\right| .
$$

We know that $f_{w}(X(p, 0, r, 0,0, u))=|p| \cdot\left|u^{\prime} r-r^{\prime} u\right|$ is a relative invariant of a regular prehomogeneous vector space whose Hessian is not identically zero, if $C \leq 2 E$ and $C$ is even $[25, \S 7$, I (13)]. Hence the linear span of $\{X(c \pm e), X(b-d)\}$ is contained in the set of the limit of $\varepsilon \operatorname{grad} \log f_{w}(X)$ $(\varepsilon, X \rightarrow 0)$, i.e., $X(c \pm e), X(b-d) \in\left(0 \times V^{*}(w)\right) \cap \mathbf{W}\left(f_{w}\right)[24,4.6]$. Since $Y_{0}$ is a linear combination of $\{X(c \pm e), X(b-d)\}, 0 \times V^{*}(w) \subset \mathbf{W}\left(f_{w}\right)$. By (8.4),

$$
\Lambda(w) \subset \mathbf{W} \text { if } C \leq 2 E \text { and } C \text { is even. }
$$

Last let us consider the case where $C>2 E$. Here we need to make explicit the pairing of $V(w)$ and $V^{*}(w)$ by which $V^{*}(w)$ is considered as the dual space of $V(w)$ : We define the pairing by $\left\langle X, X^{*}\right\rangle=\operatorname{tr}\left(X X^{*}\right)\left(X \in V(w), X^{*} \in V^{*}(w)\right)$. Then $\operatorname{grad}=\left(\frac{\partial}{\partial p_{i j}}, \frac{\partial}{\partial q_{i j}}, \cdots, \frac{\partial}{\partial t_{i j}}(i \neq j), \frac{1}{2} \frac{\partial}{\partial t_{i i}}, \frac{\partial}{\partial u_{i j}}\right)$. Put

$$
\begin{aligned}
& u_{0}=\left(1_{E}, 0_{E}, 0_{E . C-2 E}\right), \\
& r_{0}=\left(0_{E}, 1_{E}, 0_{E . C-2 E}\right), \\
& t_{0}=\operatorname{diag}\left(0_{E}, 0_{E}, 1_{C-2 E}\right) .
\end{aligned}
$$

(Here $0_{A B}$ denotes the $A \times B$-matrix whose entries are all zero, and $\left.0_{E}=0_{E \times E}.\right)$ Then, a direct calculation shows that $\lim _{\varepsilon \rightarrow 0} \varepsilon \operatorname{grad} \log f_{w}(X(\varepsilon))=$ ${ }^{t} X(1)$, for $X(\varepsilon)=X\left(\varepsilon 1_{B}, 0, \varepsilon r_{0}, 0, \varepsilon t_{0}, \varepsilon u_{0}\right)$. Since ${ }^{t} X(1) \in V^{*}(w)$ is $G(w)$-conjugate with $Y_{0}, 0 \times V^{*}(w) \subset \mathbf{W}\left(f_{w}\right)$. Thus

$$
\Lambda(w) \subset \mathbf{W} \text { if } C>2 E .
$$

Remark 8.6. In (8.5), the relative invariant $f_{w}$, is not a homogeneous polynomial except for some special cases. Hence $(G(w), V(w))$ is often non-prehomogeneous $\left[\mathbf{2 5}, \S 4\right.$, Proposition 3], although its dual $\left(G(w), V^{*}(w)\right)$ is always prehomogeneous (cf. (8.5.2)). Thus most of the prehomogeneous vector spaces $\left(G(w), V^{*}(w)\right)$, which are essential in our calculation, are not regular [25, §4, Definition 7].

8.7. $\left(D_{4}, 1,3,4\right)$

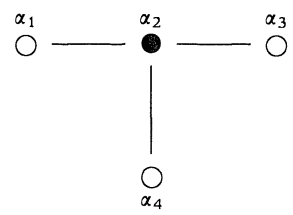


Using [12], we can show that the generalized Verma module $M\left(\lambda_{1} \varpi_{1}+\right.$ $\left.\lambda_{3} \varpi_{3}+\lambda_{4} \varpi_{4}, \mathfrak{p}\left(\left\{r_{2}\right\}\right)\right)$ is reducible if and only if $(1) \lambda_{1} \in\{0,1,2, \cdots\}$, or (2) $\lambda_{3}+\lambda_{4}$ $\in\{-2,-1,0, \cdots\}$, or (3) $\lambda_{1}+\lambda_{3}+\lambda_{4} \in\{-3,-2,-1,0, \cdots\}$, or $\left(\lambda_{1}, \lambda_{3}, \lambda_{4}\right)$ satisfies one of the conditions $\mathfrak{S}_{3}$-conjugate with (1) or (2). On the other hand, it seems that the $b$-functions coincide with the local $b$-functions at $\Lambda\left(w_{I}\right)$ and they are given by

$$
\begin{aligned}
& b_{1}\left(s_{1}, s_{3}, s_{4}\right)=\left(s_{1}+1\right)\left(s_{4}+s_{1}+3\right)\left(s_{1}+s_{3}+3\right)\left(s_{1}+s_{3}+s_{4}+4\right), \\
& b_{3}\left(s_{1}, s_{3}, s_{4}\right)=\left(s_{3}+1\right)\left(s_{1}+s_{3}+3\right)\left(s_{3}+s_{4}+3\right)\left(s_{1}+s_{3}+s_{4}+4\right), \\
& b_{4}\left(s_{1}, s_{3}, s_{4}\right)=\left(s_{4}+1\right)\left(s_{3}+s_{4}+3\right)\left(s_{4}+s_{1}+3\right)\left(s_{1}+s_{3}+s_{4}+4\right) .
\end{aligned}
$$

\section{§9. Beyond the Scalar Generalized Verma Modules}

9.0. From $[8,9.13]$ and from the examples given in the previous sections, it would be safe to assume the validity of the conjectures $A, B$, and $C$ concerning the scalar generalized Verma modules. Let us consider how they should be generalized for the generalized Verma modules which are not necessarily scalar ones.

9.1. Let notation be as in (3.1). For $\mu \in \sum_{i \in S-I} \mathbf{Z}_{\geq 0} \varpi_{i}$ and $\lambda_{d} \in \sum_{i \in S} \mathbf{Z}_{\geq 0} \varpi_{i}$, consider the functional equations of the form

$$
P(\underline{s})\left(f^{\lambda_{d}} f^{\underline{s}+\mu}\right)=b(\underline{s}) f^{\lambda_{d}} f \underline{s},
$$

where $P(\underline{s}) \in \mathscr{D}_{G}[\underline{s}]$ and $b(\underline{s}) \in \mathbf{C}[\underline{s}]$. The existence of such a functional equation with $b(\underline{s}) \neq 0$ is guaranteed by (4.1.1) or (more generally for a wider class of functions $\left.f_{i}\right)$ by [23] and [9]. Let $\mathscr{B}\left(\mu, \lambda_{d}, I\right)$ be the totality of such polynomials $b$. The author considers that Conjectures $A$ and $B$ should be generalized to the following.

$\mathbf{A}^{\prime \prime} . \mathscr{B}\left(\mu, \lambda_{d}, I\right)$ is a principal ideal of $\mathbf{C}[\underline{s}]$.

Let $b_{\mu}\left(\underline{s}+\lambda_{d}\right)=b_{\mu}\left(\underline{s}+\lambda_{d}, I\right)$ be its generator.

$\mathbf{B}^{\prime \prime}$. The generalized Verma module $M(\lambda, \mathfrak{p}(I))\left(\lambda \in \sum_{i \in S-I} \mathbf{C} \varpi_{i}+\sum_{i \in I} \mathbf{Z}_{\geq 0} \varpi_{i}\right)$ is irreducible if and only if $b_{\mu}(\lambda-\mu) \neq 0$ for any $\mu \in \sum_{i \in S-I} \mathbf{Z}_{\geq 0} \varpi_{i}$.

Remark 9.2. By $[10,6.4], \mathscr{B}(\mu, 0, I)$ is a principal ideal if Conjecture $A$ holds.

Remark 9.3. A generalized Verma module $M(\lambda, p)$ is irreducible if and only if the contravariant form [12] is non-degenerate, i.e., its discriminant is non-zero. Hence, by [12, Lemma 6, (i)], the set $\left\{\lambda_{c} \in \sum_{i \in S-I} \mathbf{C} \varpi_{i} \mid M\left(\lambda_{c}+\lambda_{d}\right)\right.$ is reducible $\}$ for a fixed $\lambda_{d} \in \sum_{i \in I} \quad \mathbb{Z}_{\geq 0} \varpi_{i}$ is a union of hypersurfaces of $\sum_{i \in S-I} \mathbf{C} \varpi_{i}$. Thus, if we assume that the irreducibility of a generalized Verma 
module is controlled by $b$-functions, it is natural to expect $A^{\prime \prime}$. The author hopes to discuss $B^{\prime \prime}$ in a different place.

Acknowledgement. The author is profitted much from conversation with A. Fujiki, to whom the author would like to express his hearty thanks.

\section{References}

[1] Alekseevsky, A. V., Component groups of centralizer for unipotent elements in semi-simple algebraic groups (in Russian), Trudy Tbilissk. Mat. Inst., 62 (1979), 5-27.

[2] Beilinson, A. and Bernstein, J., Localisation de g-modules, C.R.Acad. Sci. Paris, 292 (1981), 15-18.

[3] Bourbaki, N., Groupes et algèbres de Lie, chapitres 4, 5 et 6, Masson, 1981.

[4] Brylinski, J. L. and Kashiwara, M., Kazhdan-Lusztig conjecture and holonomic systems, Invent. Math., 64 (1981), 387-410.

[ 5] Demazure, M., Désingularisation des variétés de Schubert généralisées, Ann. Sci. Éc. Norm. Sup., 7 (1974), 53-88.

[6] Dixmier, J., Algèbres enveloppantes, Gauthier-Villards, Paris, 1974.

[7] Gyoja, A., Theory of prehomogeneous vector spaces without regularity condition, Publ. RIMS, Kyoto Univ., 27 (1991), 861-922.

[8] - Further generalization of generalized Verma modules, Publ. RIMS, Kyoto Univ., 29 (1993), 349-395.

[9] - Bernstein-Sato's polynomial for several analytic functions, J. Math. Kyoto Univ., 33 (1993), 399-411.

[10] - Local $b$-functions of prehomogeneous Lagrangians, J. Math. Kyoto Univ., 33 (1993), 413-436.

[11] Howe, R. and Umeda, T., The Capelli identity, the double commutant theorem, and multiplicity free actions, Math. Ann., 290 (1991), 565-619.

[12] Jantzen, J. C., Kontravariante Formen auf induzierten Darstellungen halbeinfacher LieAlgebren, Math. Ann., 226 (1977), 53-65.

[13] Kashiwara, M., b-Functions and holonomic systems, Intent. Math., 38 (1976), 33-53.

[14] - On the holonomic systems of linear differential equations, II, Invent. Math., 49 (1978), 121-135.

[15] -, The universal Verma module and b-function, Adr. Stud. Pure Math., 6 (1985), 67-81.

[16] Kashiwara, M. and Kawai, T., On the characteristic variety of a holonomic system with regular singularities, Adv. Math., 34 (1979), 163-184.

[17] Kashiwara, M. and Tanisaki, T., The characteristic cycles of holonomic systems on a flag manifold, Invent. Math., 77 (1984), 185-198.

[18] Kazhdan, D. and Lusztig, G., Representations of Coxeter groups and Hecke algebras, Invent. Math., 53 (1979), 165-184.

[19] - A topological approach to Springer's representations, Adr. Math., 38 (1980), $222-228$.

[20] Kimura, T., The $b$-functions and holonomy diagrams of irreducible regular prehomogeneous vector spaces, Nagoy'a Math. J., 85 (1982), 1-80.

[21] Muller, I., Rubenthaler, H. and Schiffmann, G., Structures des espaces préhomogènes associés à certaines algèbres de Lie graduées, Math. Ann., 274 (1986), 95-123.

[22] Rubenthaler, H., Espaces préhomogènes de type parabolique, Thesis (1982). 
[23] Sabbah, C., Proximité évanescente II, Compositio Math., 64 (1987), 213-241.

[24] Sato, M., Kashiwara, M., Kimura, T. and Oshima, T., Micro-local analysis of prehomogeneous vector spaces, Invent. Math., 62 (1980), 117-179.

[25] Sato, M. and Kimura, T., A classification of irreducible prehomogeneous vector spaces and their relative invariants, Nagoya Math. J., 65 (1977), 1-155.

[26] Spaltenstein, N., On the fixed point set of a unipotent element on the variety of Borel subgroups, Topology, 16 (1977), 203-204.

[27] Springer, T. A. and Steinberg, R., Conjugacy classes: Seminar on algebraic groups and related finite groups, part E, Lecture Notes in Math., 131 (1970), Springer.

[28] Steinberg, R., On the desingularization of the unipotent variety, Invent. Math., 36 (1976), 209-224.

[29] Suga, S., Highest weight modules associated with classical irreducible regular prehomogeneous vector space of commutative parabolic type, Osaka J. Math., 28 (1991), 323-346.

[30] Whitney, H., Tangents to an algebraic variety, Ann. of Math., 81 (1964), 496-549. 